\title{
Use and Validation of the Slope Determination by the Analysis of Residuals (SDAR) Algorithm
}

\author{
Enrico Lucon
}

This publication is available free of charge from: https://doi.org/10.6028/NIST.TN.2050

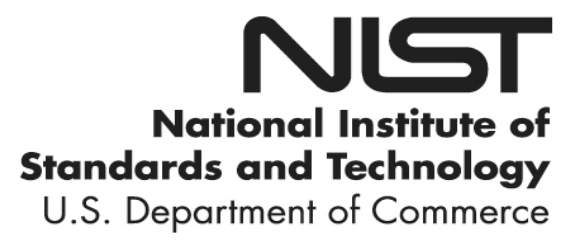




\title{
Use and Validation of the Slope Determination by the Analysis of Residuals (SDAR) Algorithm
}

\author{
Enrico Lucon \\ Applied Chemicals and Materials Division \\ Material Measurement Laboratory
}

This publication is available free of charge from:

https://doi.org/10.6028/NIST.TN.2050

June 2019

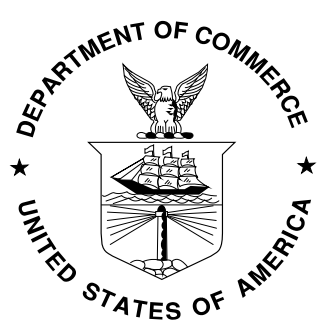

U.S. Department of Commerce

Wilbur L. Ross, Jr., Secretary

National Institute of Standards and Technology Walter Copan, NIST Director and Undersecretary of Commerce for Standards and Technology 
Certain commercial entities, equipment, or materials may be identified in this document in order to describe an experimental procedure or concept adequately. Such identification is not intended to imply recommendation or endorsement by the National Institute of Standards and Technology, nor is it intended to imply that the entities, materials, or equipment are necessarily the best available for the purpose.

National Institute of Standards and Technology Technical Note 2050

Natl. Inst. Stand. Technol. Tech. Note 2050, 45 pages (June 2019)

CODEN: NTNOEF

This publication is available free of charge from:

https://doi.org/10.6028/NIST.TN.2050 


\begin{abstract}
The Slope Determination by the Analysis of Residuals (SDAR) algorithm has recently been standardized in ASTM E3076-18 for the determination of the slope in the linear region of a test record. It offers an automated and objective linear-fitting method, based on the least normalized residual, and in its full form provides metrics for evaluating the quality of the test record and of the resultant fit.

In this investigation, SDAR was used to calculate values of crack size in elastic-plastic fracture toughness tests, conducted in accordance with ASTM E1820-18 by means of the single-specimen elastic compliance methodology.

Our analyses show that the use of SDAR is not advisable in case of noisy unloading/reloading cycles and/or in the presence of significant hysteresis, since the most linear portion identified by the algorithm may not necessarily represent the true elastic behavior of the specimen. Such issues could be alleviated by increasing the minimum number of data points used from $20 \%$ of the search range (E3076) to at least $50 \%$. Nevertheless, SDAR remains an extremely useful tool to determine a repeatable and operator-independent slope in numerous types of mechanical tests, such as tensile tests, linear elastic fracture mechanics tests, and others.
\end{abstract}

\title{
Key words
}

Analysis of residuals, ASTM E1820, ASTM E3076, elastic compliance, linear fitting, mechanical tests, SDAR, slope determination. 


\section{Table of Contents}

1. Introduction ........................................................................................................................... 1

2. "Full” SDAR algorithm...................................................................................... 2

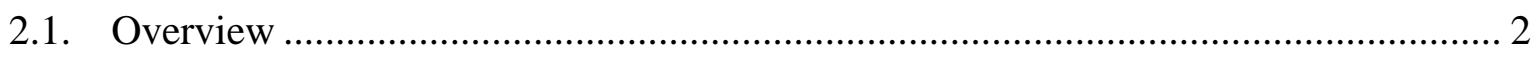

2.1.1. Offset, truncation and normalization of the test record....................................... 2

2.1.2. First data quality metric: noise in the data ………...................................... 3

2.1.3. Second data quality metric: digital resolution ................................................ 3

2.1.4. Establishment and linear regression of the most linear part of the data set .......... 4

2.1.5. First fit quality metric: curvature in the vicinity of the fit range............................ 5

2.1.6. Second fit quality metric: size of the linear region ............................................. 5

2.1.7. Un-normalization of the slope and intercept ....................................................... 5

2.2. Implementation of the "full" SDAR algorithm: SDAR linear fitting.xIsm Excel

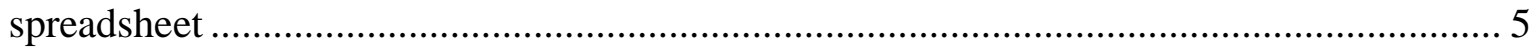

2.3. Spreadsheet validation.................................................................................... 12

2.3.1. ASTM E3076 example tensile data set: File 01 ................................................. 12

2.3.2. ASTM E08.03 analytical round-robin for the validation of the SDAR algorithm 13

3. "Stripped-down" version of the SDAR algorithm for the measurement of elastic compliance: Compliance calculation.xlsm spreadsheet.............................................................. 16

3.1. "Regression" Visual Basic subroutine ……………............................................. 18

4. ASTM E1820-18: recommended best practices for calculating elastic compliance 18

4.1. Use of the SDAR algorithm for compliance calculations on actual fracture toughness data sets

4.1.1. $J-R$ curve tests on $1 \mathrm{TC}(\mathrm{T})$ specimens: round-robin organized by HZDR (Germany, 2012).

4.1.2. ASTM round-robin on the analysis of standard data sets for fracture toughness evaluation (2013) .......................................................................................................... 27

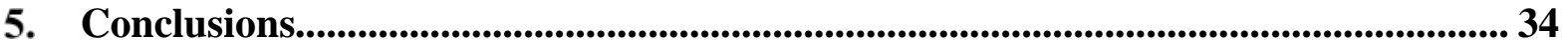

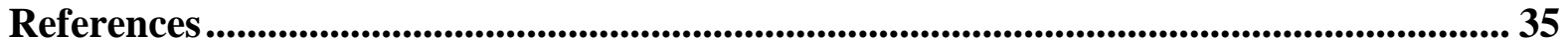

Appendix A: Visual Basic code of the Excel subroutine "Regression” ............................... 36

\section{List of Tables}

Table 1. Comparison between ASTM E3076-18 and Excel spreadsheet for File 01.

Table 2. Comparison between SDAR round-robin (ASTM r-r) and Excel (NIST) analysis results (Part 1: regression results). Relative differences greater than $2 \%$ are highlighted in bold red. 
Table 3. Comparison between SDAR round-robin (ASTM r-r) and Excel (NIST) analysis results (Part 2: resolution, noise, tail slopes, and relative fit range). Acceptable results are highlighted in green, unacceptable in red.

Table 4. Overall comparisons for the 1TC(T) specimens tested for the HZDR round-robin.

Table 5. Absolute differences for initial crack size and total crack extension.

Table 6. Relative spread of the plane-strain toughness values $J_{Q} / J_{I c}$.

Table 7. Assessment of SDAR analyses with respect to the original NIST submission for the 2013 ASTM round-robin.

Table 8. Assessment of SDAR analyses with respect to mean participants' results from the 2013 ASTM round-robin.

\section{List of Figures}

Fig. 1. Tangent construction used to truncate data set (from ASTM E3076-18).

Fig. 2. Sheet "Input data".

Fig. 3. Sheet "Shifted curve".

Fig. 4. Sheet "Tangent construction".

Fig. 5. Sheet "Normalized record".

Fig. 6. Sheet "Signal noise check".

Fig. 7. Sheet "Digital resolution check".

Fig. 8. Sheet "Regression".

Fig. 9. Sheet "Fitting results".

Fig. 10. Sheet "Assessment of Fit Quality".

Fig. 11. Results printout.

Fig. 12. Plot of the original test record File 01 (from ASTM E3076-18).

Fig. 13. Spreadsheet Compliance calculation.xlsm.

Fig. 14. Comparison between $J_{Q}$ calculated per ASTM E1820 App. X3, by fitting all data, and using the SDAR algorithm. $\pm 20 \%$ indicates the typical scatter band of $J_{I c}$ values.

Fig. 15. Comparison between measured $a_{0}$ and $a_{0 q}$ calculated according to ASTM E1820 App. X3, by fitting all data, and by means of SDAR. $\pm 1 \% \mathrm{~W}$ is the ASTM requirement. Fig. 16. Comparison between $\Delta a_{p}$ and $\Delta a_{\text {pred }}$ obtained according to ASTM E1820 App. X3, by fitting all data, and by means of SDAR. $\pm 15 \% \Delta a_{p}$ is the ASTM requirement.

Fig. 17. Differences between predicted and measured initial crack sizes, and comparison with the ASTM E1820 requirement $( \pm 1 \% \mathrm{~W})$.

Fig. 18. Differences between predicted and measured total crack extensions, and comparison with the ASTM E1820 requirement $\left( \pm 15 \% \Delta a_{p}\right)$.

Fig. 19. Values of spread between minimum and maximum $J_{Q}$ obtained by the three approaches used (E1820 App. X3, fitting all data, SDAR algorithm).

Fig. 20. Worst agreement: BBL-CGW_24, UallRall ( $J_{Q}$ spread: $32.2 \%$ ).

Fig. 21. Best agreement: BBL-CGW_25, UallRall ( $J_{Q}$ spread: $1.2 \%$ ).

Fig. 22. Worst agreement: FYB-A1.

Fig. 23. Unsatisfactory agreement: GJO-12A.

Fig. 24. Best agreement: EUR-U26.

Fig. 25. Pronounced noise and hysteresis: maximum force unloading cycle for FYB-A1.

Fig. 26. Significant noise and hysteresis: maximum force unloading cycle for GJO-12A.

Fig. 27. Low noise and hysteresis: maximum force unloading cycle for EUR-U26. 
Fig. 28 - Specimen BBL-CGW_23 (HZDR round-robin): comparison between ASTM E1820 App. X3 analysis and SDAR analyses (with $20 \%$ and $50 \%$ minimum window size).

Fig. 29. Specimen FYB-A1: percentage of unloading cycle data points used by SDAR.

Fig. 30. Specimen EUR-U26: percentage of unloading cycle data points used by SDAR.

Fig. 31. Comparison between critical toughness values for the ASTM round-robin data sets.

Fig. 32. Comparison between measured and predicted values of final crack extension for the ASTM round-robin data sets. 


\section{Introduction}

In mechanical testing, it is often required that the linear portion of a force/displacement test record be identified and analyzed. For example:

- In tensile testing, the slope of the elastic portion of the stress/strain curve provides an indication of the Young's modulus and is required for the determination of the yield strength at the intersection between the curve and the offset line corresponding to $0.2 \%$ permanent deformation [1].

- In Linear Elastic Fracture Mechanics (LEFM) testing, the calculation of the plane-strain fracture toughness, $K_{I c}$, requires linearly fitting the initial portion of the force vs. loadline displacement (or crack-mouth opening displacement) record [2].

- In elastic-plastic (EPFM) fracture toughness testing, the use of the elastic compliance single-specimen method for calculating crack sizes requires the determination of the slope of several unloading/reloading cycles throughout the test [3]. The elastic (or unloading) compliance method is also commonly used in fatigue crack growth testing [4].

Several difficulties can be encountered when performing linear regressions of test data, such as very limited portions of the data exhibiting linear behavior, or data deviating very gradually from linearity. In many instances, early non-linearities in the test record have to be accounted for in the analyses. Sometimes, small differences in the identification of the linear portion of the same test record by different users can lead to significant discrepancies in the final results.

Ideally, a specific data set should lead different analysts to obtain the same results, if the slope can be accurately and repeatably determined without the need for subjective operator input.

Several authors have proposed algorithms for determining the linear range in a test record [5-6]. In 2004, Scibetta and Schuurmans [7] addressed the question regarding how to determine the number of points to be used in the linear fit, below a knee where slope shows a significant decrease. In their algorithm, an iterative process of successive linear regressions on varying window sizes is used to search for the window that corresponds to the lowest normalized residual. The main limitation of this approach is that, for batch processing of multiple large data sets, it can take considerable computational time to perform all the regressions.

In 2011, Graham and Adler [8] presented an optimized algorithm that performs a fully automated determination of the slope in the linear range of a data set and includes criteria for determining the quality of the data and the quality of the linear fit. Both criteria are intended to assess whether the record has indeed a linear range. This algorithm, which was denominated SDAR (Slope Determination by Analysis of Residuals), uses the analysis of residuals to determine the data to be used in the fit and how the data deviate from the fit at the ends of the fit range.

In 2016, an ASTM Work Item (WK55299) was initiated inside ASTM Sub-Committee E08.03 (Advanced Apparatus and Techniques), under the leadership of S. Graham (US Naval Academy), aimed at creating a new standard, with the title "Practice for Determination of Slope in the Linear Region of a Test Record.” The ASTM standard was 
successfully balloted in November 2018, and was finally published in April 2019 with the number ASTM E3076-18.

This Technical Note describes the implementation of the SDAR algorithm into a macro-enabled Microsoft Excel ${ }^{1}$ spreadsheet, as well as its application (and validation) on several data sets used by ASTM E08.03 members for an analytical round-robin. In addition, a simplified version of SDAR (which excludes the determination of the quality of data and the quality of fit) was implemented into another Excel Visual Basic routine, to be used for the determination of crack size from unloading/reloading slope measurement. The results of this analytical approach were compared to conventional elastic compliance measurements for several fracture toughness data sets, and conclusions on the applicability of SDAR for compliance determination were drawn.

\section{2. “Full” SDAR algorithm}

\subsection{Overview}

The content of this Section is extracted from the ASTM E3076-18 standard.

The various steps of the procedure can be summarized as follows.

(a) The range of the test record to be searched for the most linear region is established, by determining if there is a local maximum or "knee" above which the slope shows a significant decrease.

(b) The test record is offset and normalized so that quality metrics can be applied that are independent of scale and engineering units.

(c) Metrics on noise level and digital resolution are applied to evaluate the quality of the data set.

(d) The most linear region of the test record is numerically determined by the analysis of residuals, using a tangency point approach to truncate the test record.

(e) The quality of the resulting linear fit is evaluated by means of analysis of residuals.

If the data set fails one or more of the quality metrics, the analyst is encouraged to decide whether the test record is adequate with respect to the application or calling method.

\subsubsection{Offset, truncation and normalization of the test record}

The data set is first offset by subtracting the $x$-value (xshift) and $y$-value (yshift) of the first data point from the $x$-values and $y$-values of all data points.

Then, the point with coordinates $\left(x_{1}, y_{1}\right)$ is found in the shifted test record, where the $y$-value just exceeds $5 \%$ of the maximum $y$-value in the shifted data set $\left(y_{\max }\right)$.

An offset point (xoffset, yoffset) is created with xoffset $=x_{1}$ and yoffset $=y_{1}+0.15$. $y_{\max }$. Starting with the first point where $y>$ yoffset, one must find the point where the line

\footnotetext{
${ }^{1}$ Certain commercial products are identified in this article in order to describe the experimental procedure adequately. Such identification is not intended to imply recommendation or endorsement by the National Institute of Standards and Technology, nor is it intended to imply that the products identified are necessarily the best available for the purpose.
} 
drawn from that point to the offset point has the largest slope. This is the tangency point (xtangent, ytangent), see Fig. 1.

All the points beyond the tangent point are truncated and all the points below the tangent point are normalized by dividing their $y$-values and $x$-values by the corresponding values for the tangency point. The resulting normalized and truncated data set has now a range from 0 to 1 in both $x$ and $y$.

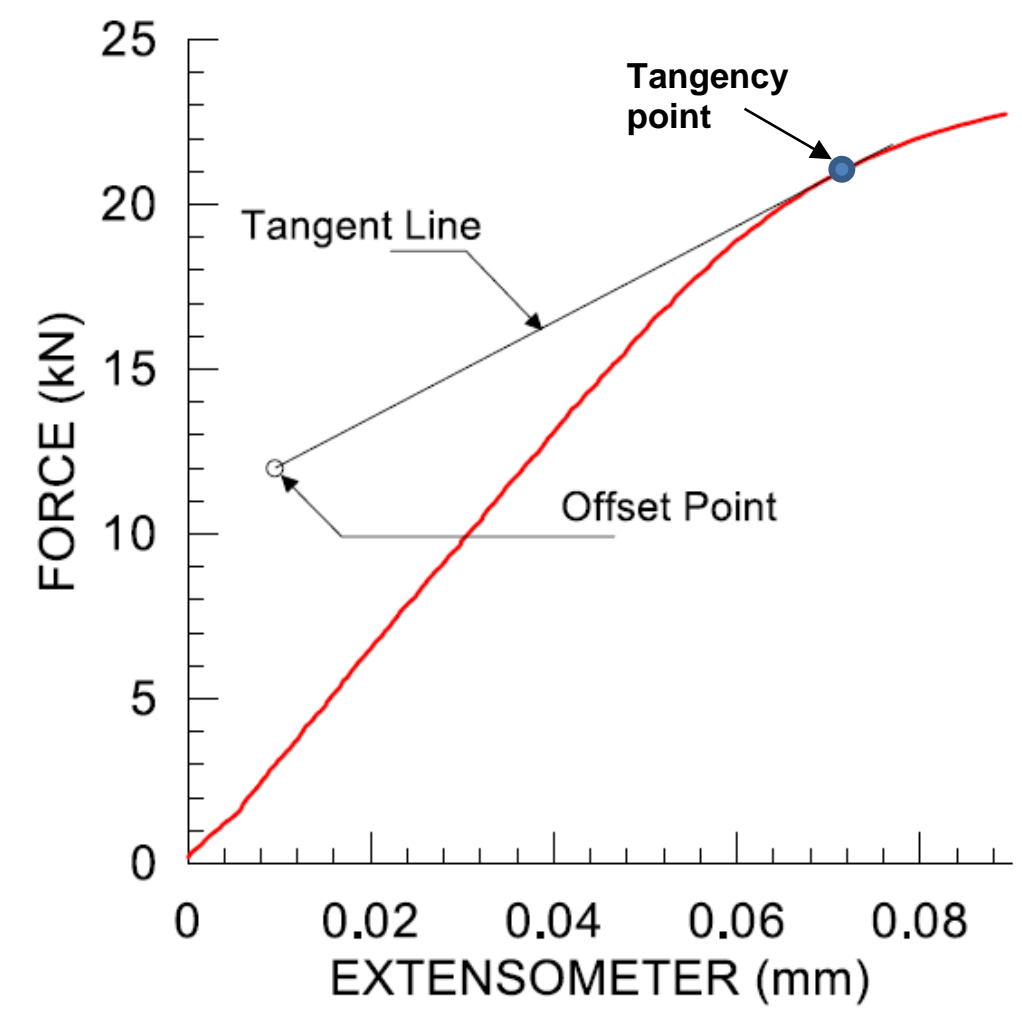

Fig. 1. Tangent construction used to truncate data set (from ASTM E3076-18).

\subsubsection{First data quality metric: noise in the data}

The allowable normalized noise in the data is 0.005 , which corresponds to $0.5 \%$ of the $x$ and $y$ values at the tangency point.

The normalized $x$ noise is the standard deviation of the $\Delta x r_{i}$ values, where:

$$
\Delta x r_{i}=\Delta x_{i}-\overline{\Delta x} \quad \text { for } i=1 \text { to } N-1,
$$

with $\Delta x_{i}=x_{i+1}-x_{i}$, and $\overline{\Delta x}$ is the mean value of $\Delta x_{i} . N$ is the number of points in the truncated data set. The normalized $y$ noise is calculated in the same way.

\subsubsection{Second data quality metric: digital resolution}

The optimum digital resolution, $\delta$, is $2^{-12}$.

For the $x$ variable, calculate first: 


$$
\Delta \Delta x_{i}=\left|\Delta x_{i+1}-\Delta x_{1}\right| \quad \text { for } i=1 \text { to } N-2 \text {, }
$$

then generate a histogram of $\Delta \Delta x$ using bin start-points of $-\delta / 2+j \delta$ and bin end-points of $\delta / 2$ $+j \delta$, where $j$ starts at zero.

Calculate next the percent of the data in the zeroth bin $(j=0)$ by counting the points with $-\delta / 2$ $\leq \Delta \Delta x \leq \delta / 2$, and dividing by $N-2$. For the remaining bins $(j>0)$, the percent is calculated by dividing the number of points in the bin by $N-2$.

Excluding the zeroth bin, let $z$ be the bin number with the highest percent. The requirements of ASTM E3076 on digital resolution are:

- $0<z \leq 3$,

- $\quad$ percent in bin $z \leq 25 \%$, and

- $\quad$ percent in zeroth bin $\leq 25 \%$.

If any of the above requirements are not met, the digital resolution may be insufficient.

The same steps shall be repeated for the normalized $y$ values.

\subsubsection{Establishment and linear regression of the most linear part of the data set}

The tangency point previously determined represents the upper end of the data used in the search of the linear portion. The first window starts with the first data point and includes the larger between 10 points or $20 \%$ of $N$.

After performing a linear regression, the normalized residual is calculated as:

$$
\text { NormResidual }=\frac{\sum\left(y_{i}-m x_{i}-b\right)^{2}}{\sum\left(y_{i}-\bar{y}\right)^{2}} \quad,
$$

where $m$ and $b$ are slope and intercept of the linear regression, respectively, and $\bar{y}$ is the mean value of $y$ in the current search window.

The window height is then increased by one point, the linear regression is repeated, and the normalized residual is calculated. The process is repeated until the upper index of the search window coincides with the top of the search range. Next, the lower index of the window is incremented by one point, and the whole process is repeated until the size of the window is the larger between $20 \%$ of $N$ or 10 points.

Once this iterative process is concluded, the window corresponding to the lowest normalized residual is the optimum (most linear) region of the test record.

Note that, if the upper index of the optimum window coincides with the last point in the truncated test record, the most linear region may be cut off by the tangency point. In this case, the offset point in Sec. 2.1.1 must be raised by $5 \%$ of $y_{\max }$, and the analysis should be repeated until the upper index does not correspond to the last point of the data set.

The total number of linear fits performed is given by:

$$
N_{\text {fits }}=0.5\left[\left(n-N_{\text {min }}+1\right)^{2}+\left(n-N_{\text {min }}+1\right)\right],
$$

where $n$ is the number of points in the search range and $N_{\min }$ is the larger between $20 \%$ of $N$ and 10 points. 


\subsubsection{First fit quality metric: curvature in the vicinity of the fit range}

The normalized $y$ residuals, calculated as:

$$
\text { yResidual }{ }_{i}=y_{i}-\left(m x_{i}+b\right) \quad,
$$

are plotted as a function of normalized $x$. The set of residuals is divided into four quartiles between $x_{\min }$ and $x_{\max }$, which correspond to the minimum and maximum of the normalized $x$ values for the optimum region. Each quartile includes $1 / 4$ of the range between $x_{\min }$ and $x_{\max }$.

Calculate the slope of the residual in the $1^{\text {st }}$ and $4^{\text {th }}$ quartiles (as long as the number of points in each quartile is at least 5). The residual slope must not exceed $5 \%$ of the fitted slope calculated in Sec. 2.1.4.

\subsubsection{Second fit quality metric: size of the linear region}

The difference between minimum and maximum normalized $y$-values of the final fit data set must be at least $40 \%$ of the $y$-value of the tangency point. If the range is less than $40 \%$, the linear region may be unacceptably small.

\subsubsection{Un-normalization of the slope and intercept}

The slope is un-normalized using:

$$
m_{\text {final }}=m \frac{\text { ytangent }}{x \text { tangent }} .
$$

The intercept is un-normalized using:

$$
b_{\text {final }}=b \cdot \text { ytangent } \quad \text {, }
$$

To remove the effect of the data shift from Sec. 2.1.1, the following equation is used:

$$
b_{\text {true }}=b_{\text {final }}-x \text { shift } \cdot m_{\text {final }}+y \text { shift } .
$$

The lower and upper bounds of the final fit range are given by:

$$
\begin{aligned}
& y \text { LowerBound }=y_{\min } \cdot y \text { tangent }+y \text { shift } \\
& y \text { UpperBound }=y_{\max } \cdot y \text { tangent }+y \text { shift } .
\end{aligned}
$$

\subsection{Implementation of the "full" SDAR algorithm: SDAR linear fitting.xIsm Excel spreadsheet}

The complete SDAR algorithm, as described in Sec. 2.1, was implemented in an Excel macro-enabled spreadsheet called SDAR linear fitting.xIsm (freely available from the author, enrico.lucon@nist.gov).

The complete $x-y$ data set is input in columns B and C (highlighted in green) of the sheet "Input data" (Fig. 2). If data are already present, columns B and C can be emptied by clicking on "Clear data". The test record, shifted in accordance with Sec. 2.1.1, is visualized in the sheet "Shifted curve" (Fig. 3). The corresponding $x$ and $y$ values are shown in columns $\mathrm{H}$ and $\mathrm{I}$. 
Columns P and Q contain the truncated data set, and columns R and S present the normalized data set. NOTE: the user must adjust the length of columns $\mathrm{H}$ to $\mathrm{S}$ to match the number of rows in columns $\mathrm{B}$ and $\mathrm{C}$.

Additional input from the user is required in Cell F9 (highlighted in yellow), corresponding to the offset, expressed in percent of the maximum $y$-value. Should the analysis be repeated as indicated in Sec. 2.1.4, the user would input $10 \%, 15 \%$, etc. (incrementing the offset value by $5 \%$ ).

\begin{tabular}{|c|c|c|c|c|c|c|c|c|c|c|c|c|c|c|c|c|c|c|c|c|}
\hline 4 & A & 8 & c & D & E & F & G & H & I & J & $\kappa$ & L & M & $\mathrm{N}$ & 0 & $p$ & a & $\mathbf{R}$ & $s$ & $T$ \\
\hline 1 & Index & $x$ & $\gamma$ & Clear & $x_{\text {sinth }}=$ & $5.8 \mathrm{E}-05$ & & $x_{\text {snitined }}$ & $\mathbf{Y}_{\text {shitited }}$ & \multicolumn{2}{|c|}{$>5 \% \operatorname{Max} y$} & Slope & itancents & $\mathrm{x}_{\text {tansente }}$ & Ytancent & $x_{\text {eruncate }}$ & $Y_{\text {truncoste }}$ & $x_{\text {norm }}$ & Yoserm & \\
\hline 2 & 1 & 5.8E- 05 & 0.19138 & data & $\gamma_{\text {simin }}=$ & 0.19138 & & 0 & 0 & FALSE & FALSE & & & & & 0 & 0 & 0 & 0 & \\
\hline 3 & 2 & $6.4 E-05$ & 0.19154 & & $\operatorname{Max} y=$ & 59.9727 & & 5.7811E-06 & 0.00017 & FALSE & FALSE & & & & & $5.7811 \mathrm{E}-06$ & 0.00017 & $8.3 E-05$ & $8 E-06$ & \\
\hline 4 & 3 & 4.3E-05 & 0.19273 & & $\% \operatorname{Maxy}=$ & 2.99864 & & $-1.46982 \mathrm{E}-05$ & 0.00136 & FALSE & FALSE & & & & & $-1.46982 \mathrm{E}-05$ & 0.00136 & -0.00021 & 6.6E-05 & \\
\hline 5 & 4 & 0.00015 & 0.20929 & & & & & 8.75018E-05 & 0.01791 & FALSE & FALSE & & & & & $8.75018 \mathrm{E}-05$ & 0.01791 & 0.00125 & 0.00087 & \\
\hline 6 & 5 & 0.00021 & 0.25656 & & $x_{1}=$ & 0.01016 & & 0.000155603 & 0.06518 & FALSE & FALSE & & & & & 0.000155603 & 0.06518 & 0.00223 & 0.00316 & \\
\hline 7 & 6 & 0.00044 & 0.33308 & & $\gamma_{1}=$ & 3.012 & & 0.000386263 & 0.1417 & FALSE & FALSE & & & & & 0.000386263 & 0.1417 & 0.00553 & 0.00688 & \\
\hline 8 & 7 & 0.00088 & 0.4332 & & & & & 0.000824458 & 0.24182 & FALSE & FALSE & & & & & 0.000824458 & 0.24182 & 0.0118 & 0.01174 & \\
\hline 9 & 8 & 0.00145 & 0.55334 & & Offset = & $15 \%$ & $\max y$ & 0.001393857 & 0.36196 & FALSE & FALSE & & & & & 0.001393857 & 0.36196 & 0.01994 & 0.01757 & \\
\hline 10 & 9 & 0.00186 & 0.68653 & & $x_{\text {ortser }}=$ & 0.01016 & & 0.001806576 & 0.49515 & FALSE & FALSE & & & & & 0.001806576 & 0.49515 & 0.02585 & 0.02403 & \\
\hline 11 & 10 & 0.00235 & 0.82218 & & Yolrser $=$ & 12.0083 & & 0.002295039 & 0.6308 & False & FALSE & & & & & 0.002295039 & 0.6308 & 0.03284 & 0.03062 & \\
\hline 12 & 11 & 0.00292 & 0.95098 & \multirow{3}{*}{\multicolumn{2}{|c|}{ Max slope = }} & & & 0.00285944 & 0.7596 & FALSE & FALSE & & & & & 0.00285944 & 0.7596 & 0.04091 & 0.03687 & \\
\hline 13 & 12 & 0.0035 & 1.0705 & & & 143.886 & & 0.003446379 & 0.87912 & FALSE & FALSE & & & & & 0.003446379 & 0.87912 & 0.04931 & 0.04267 & \\
\hline 14 & 13 & 0.00385 & 1.18194 & & & & & 0.003790899 & 0.99056 & FALSE & FALSE & & & & & 0.003790899 & 0.99056 & 0.05424 & 0.04808 & \\
\hline 15 & 14 & 0.00434 & 1.28654 & & $x_{\text {consem }}=$ & 0.06989 & & 0.004286025 & 1.09516 & FALSE & FALSE & & & & & 0.004286025 & 1.09516 & 0.06133 & 0.05316 & \\
\hline 16 & 15 & 0.00488 & 1.3877 & & $V_{\text {rarsenent }}=$ & 20.6019 & & 0.004817307 & 1.19633 & FALSE & FALSE & & & & & 0.004817307 & 1.19633 & 0.06893 & 0.05807 & \\
\hline 17 & 16 & 0.00527 & 1.48836 & & itroreen $=$ & 220 & & 0.005212976 & 1.29698 & FALSE & FALSE & & & & & 0.005212976 & 1.29698 & 0.07459 & 0.06295 & \\
\hline 18 & 17 & 0.00567 & 1.58679 & & & & & 0.005615506 & 1.39541 & FALSE & FALSE & & & & & 0.005615506 & 1.39541 & 0.08035 & 0.06773 & \\
\hline 19 & 18 & 0.00589 & 1.68479 & & 0.01016 & 12.0083 & & 0.005836856 & 1.49341 & FALSE & FALSE & & & & & 0.005836856 & 1.49341 & 0.08352 & 0.07249 & \\
\hline 20 & 19 & 0.00602 & 1.78309 & & 0.06989 & 20.6019 & & 0.005965216 & 1.59171 & FALSE & FALSE & & & & & 0.005965216 & 1.59171 & 0.08535 & 0.07726 & \\
\hline 21 & 20 & 0.00628 & 1.88034 & & & & & 0.006218221 & 1.68896 & FALSE & FALSE & & & & & 0.006218221 & 1.68896 & 0.08897 & 0.08198 & \\
\hline 22 & 21 & 0.00652 & 1.97674 & & & & & 0.006462011 & 1.78536 & FALSE & FALSE & & & & & 0.006462011 & 1.78536 & 0.09246 & 0.08666 & \\
\hline 23 & 22 & 0.00684 & 2.07144 & & $N=$ & 220 & & 0.006781836 & 1.88007 & FALSE & FALSE & & & & & 0.006781836 & 1.88007 & 0.09704 & 0.09126 & \\
\hline 24 & 23 & 0.00714 & 2.16572 & & $i_{\text {stant }}=$ & & & 0.007081281 & 1.97435 & FALSE & FALSE & & & & & 0.007081281 & 1.97435 & 0.10132 & 0.09583 & \\
\hline 25 & 24 & 0.00746 & 2.26063 & & $i_{\text {end }}=$ & 220 & & 0.007401896 & 2.06925 & FALSE & FALSE & & & & & 0.007401896 & 2.06925 & 0.10591 & 0.10044 & \\
\hline 26 & 25 & 0.00775 & 2.35575 & & & & & 0.007697031 & 2.16437 & FALSE & FALSE & & & & & 0.007697031 & 2.16437 & 0.11013 & 0.10506 & \\
\hline 27 & 26 & 0.00799 & 2.45066 & & $5=$ & 0.00024 & & 0.007929161 & 2.25928 & FALSE & FALSE & & & & & 0.007929161 & 2.25928 & 0.11345 & 0.10966 & \\
\hline 28 & 27 & 0.00823 & 2.54564 & & & & & 0.008169616 & 2.35426 & FALSE & FALSE & & & & & 0.008169616 & 2.35426 & 0.11689 & 0.11427 & \\
\hline 29 & 28 & ก กกหร7 & J fanaq & & & 44 & & กอ851 3ก61 & 204911 & alst & 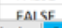 & & & & & ก กกะร1 2061 & 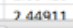 & 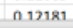 & 28 & \\
\hline
\end{tabular}

Fig. 2. Sheet "Input data".

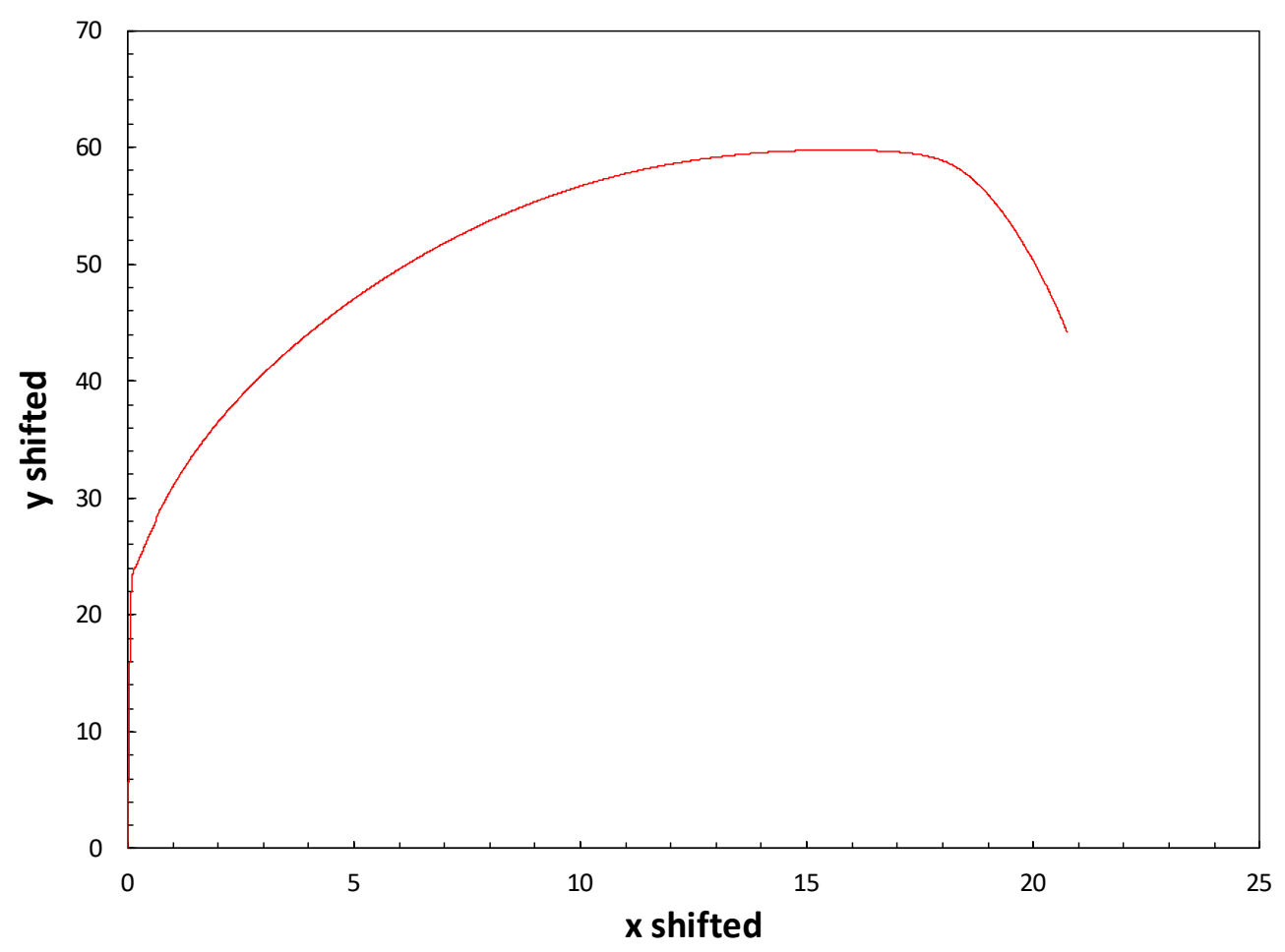

Fig. 3. Sheet "Shifted curve". 
The establishment of the tangency point and the obtained normalized record are shown in the sheets "Tangent construction" (Fig. 4) and "Normalized record" (Fig. 5), respectively.

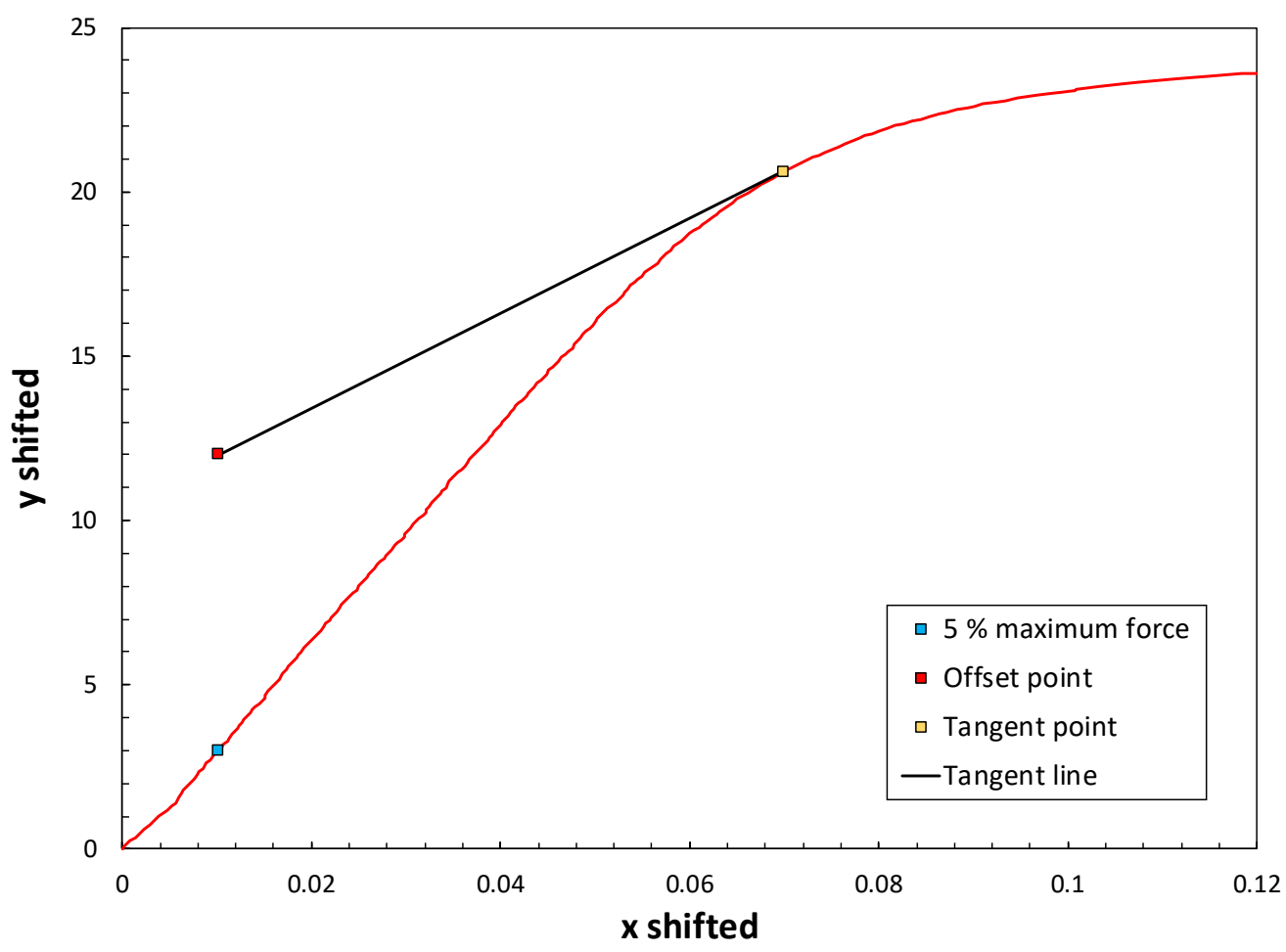

Fig. 4. Sheet "Tangent construction".

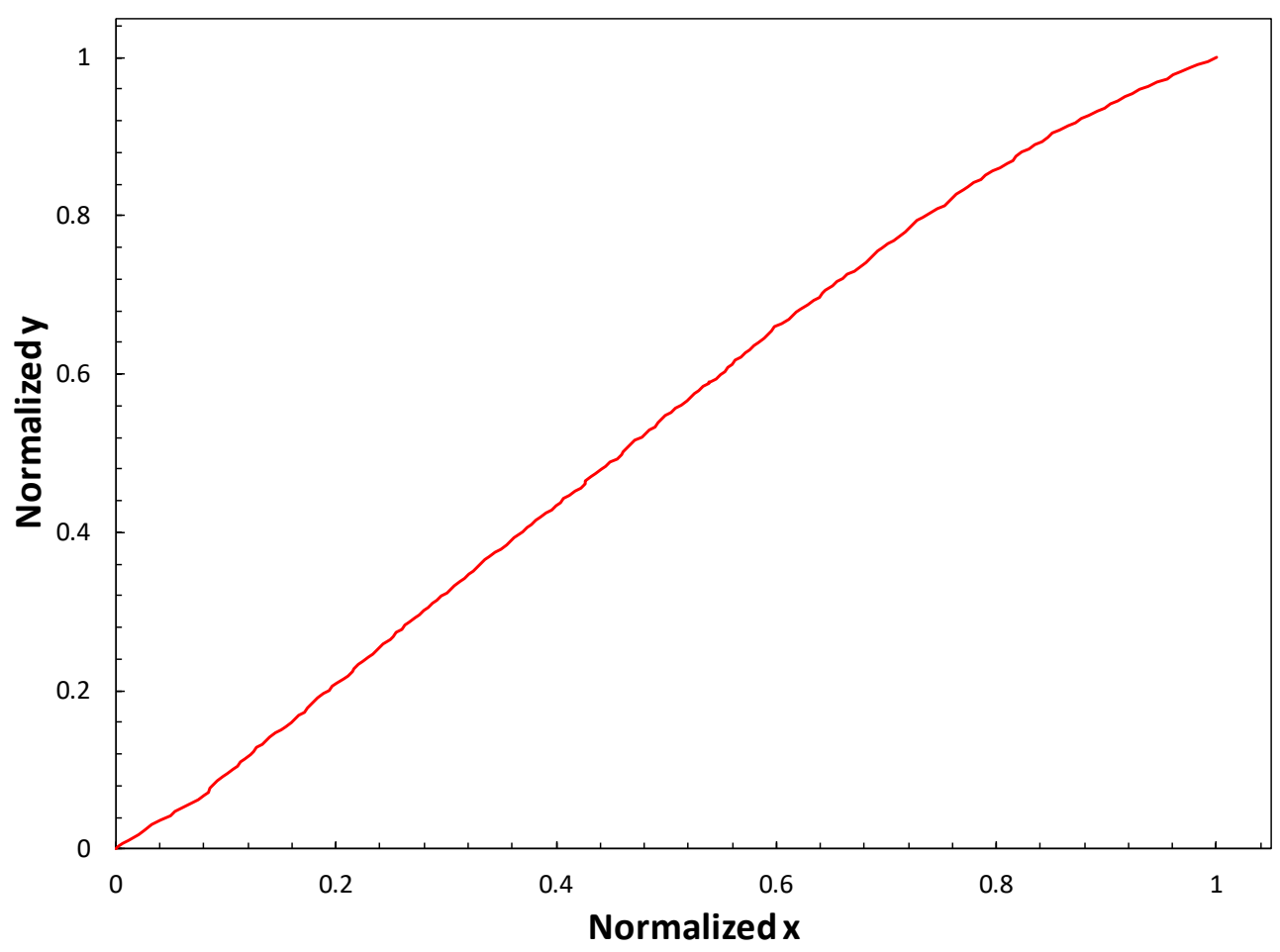

Fig. 5. Sheet "Normalized record". 
The first data quality metric (signal noise, Sec. 2.1.2) is implemented in the sheet "Signal noise check" (Fig. 6). The sheet is capable of handling up to 2000 data points, but it can be easily modified to accommodate a larger number. Normalized data points are automatically copied from columns R and S of the sheet "Input data”.

The relative noise for $x$ and $y$ is provided in cells F8 and F9, and is obtained by dividing the normalized noise (cells F6 and F7) by the ASTM E3076-18 limit (0.005, or 0.5 \%). If the values in cells F8 and F9 are less than 1 (i.e., noise in cells F6 and F7 lower than 0.005), the cells are highlighted in green; otherwise, they are highlighted in red (indicating unacceptable noise levels).

\begin{tabular}{|c|c|c|c|c|c|c|c|c|c|c|c|c|c|c|c|c|c|c|c|c|}
\hline 4 & A & B & c & D & E & $\mathrm{F}$ & G & H & 1 & J & k & L & M & $\mathrm{N}$ & 0 & $p$ & Q & $R$ & s & $T$ \\
\hline 1 & i & $x_{\text {nomm }}$ & $Y_{\text {norm }}$ & & $N=$ & 220 & & $\Delta x_{i}$ & $\Delta y_{1}$ & $\Delta x x_{1}$ & $\Delta \mathrm{yr}_{\mathrm{i}}$ & & & & & & & & & \\
\hline 2 & 1 & 0 & 0 & & & & & 8.2718E-05 & 8.043E-06 & -0.0044835 & -0.0045582 & & & & & & & & & \\
\hline 3 & 2 & 8. $3 E-05$ & $8 E-06$ & & $\overline{\Delta x_{i}}=$ & 0.00457 & & -0.000293 & $5.7728 \varepsilon-05$ & -0.0048592 & -0.0045085 & & & & & & & & & \\
\hline 4 & 3 & -0.00021 & $6.6 \mathrm{E}-05$ & & $\overrightarrow{\Delta y_{t}}=$ & 0.00457 & & 0.00146231 & 0.00080366 & -0.0031039 & -0.0037625 & & & & & & & & & \\
\hline 5 & 4 & 0.00125 & 0.00087 & & & & & 0.00097441 & 0.00229438 & -0.0035918 & -0.0022718 & & & & & & & & & \\
\hline 6 & 5 & 0.00223 & 0.00316 & Norm. & noise $x=$ & 0.00163 & & 0.00330036 & 0.00371409 & -0.0012658 & -0.0008521 & & & & & & & & & \\
\hline 7 & 6 & 0.00553 & 0.00688 & Norm. & - noise $y=$ & 0.00072 & & 0.00626984 & 0.00486009 & 0.00170363 & 0.00029388 & & & & & & & & & \\
\hline 8 & 7 & 0.0118 & 0.01174 & & & & & 0.00814715 & 0.00583144 & 0.00358094 & 0.00126523 & & & & & & & & & \\
\hline 9 & 8 & 0.01994 & 0.01757 & Relative & enoise $x=$ & 0.325 & & 0.00590532 & 0.00646478 & 0.00133911 & 0.00189857 & & & & & & & & & \\
\hline 10 & 9 & 0.02585 & 0.02403 & Relative & e noise y $=$ & 0.144 & & 0.00698909 & 0.00658443 & 0.00242288 & 0.00201822 & & & & & & & & & \\
\hline 11 & 10 & 0.03284 & 0.03062 & & & & & 0.00807564 & 0.00625162 & 0.00350943 & 0.00168541 & & & & & & & & & \\
\hline 12 & 11 & 0.04091 & 0.03687 & & & & & 0.00839812 & 0.00580176 & 0.00383191 & 0.00123555 & & & & & & & & & \\
\hline 13 & 12 & 0.04931 & 0.04267 & & & & & 0.00492951 & 0.00540888 & 0.0003633 & 0.00084267 & & & & & & & & & \\
\hline 14 & 13 & 0.05424 & 0.04808 & & & & & 0.00708443 & 0.00507741 & 0.00251822 & 0.0005112 & & & & & & & & & \\
\hline 15 & 14 & 0.06133 & 0.05316 & & & & & 0.00760176 & 0.00491048 & 0.00303555 & 0.00034427 & & & & & & & & & \\
\hline 16 & 15 & 0.06893 & 0.05807 & & & & & 0.00566136 & 0.00488572 & 0.00109515 & 0.00031951 & & & & & & & & & \\
\hline 17 & 16 & 0.07459 & 0.06295 & & & & & 0.00575953 & 0.00477772 & 0.00119332 & 0.00021151 & & & & & & & & & \\
\hline 18 & 17 & 0.08035 & 0.06773 & & & & & 0.00316715 & 0.00475666 & -0.0013991 & 0.00019045 & & & & & & & & & \\
\hline 19 & 18 & 0.08352 & 0.07249 & & & & & 0.00183662 & 0.00477141 & -0.0027296 & 0.0002052 & & & & & & & & & \\
\hline 20 & 19 & 0.08535 & 0.07726 & & & & & 0.00362008 & 0.00472054 & -0.0009461 & 0.00015433 & & & & & & & & & \\
\hline 21 & 20 & 0.08897 & 0.08198 & & & & & 0.00348823 & 0.00467909 & 0.001078 & 0.00011288 & & & & & & & & & \\
\hline 22 & 21 & 0.09246 & 0.08666 & & & & & 0.00457616 & 0.00459711 & 9.9534E-06 & $3.0899 \mathrm{E}-05$ & & & & & & & & & \\
\hline 23 & 22 & 0.09704 & 0.09126 & & & & & 0.00428456 & 0.00457629 & -0.0002817 & $1.0076 \mathrm{E}-05$ & & & & & & & & & \\
\hline 24 & 23 & 0.10132 & 0.09583 & & & & & 0.00458747 & 0.00460653 & $2.1257 \mathrm{E}-05$ & $4.0316 \mathrm{E}-05$ & & & & & & & & & \\
\hline 25 & 24 & 0.10591 & 0.10044 & & & & & 0.00422289 & 0.00461735 & -0.0003433 & $5.114 E-05$ & & & & & & & & & \\
\hline 26 & 25 & 0.11013 & 0.10506 & & & & & 0.00332139 & 0.00460657 & -0.0012448 & 4.0364E-05 & & & & & & & & & \\
\hline 27 & 26 & 0.11345 & 0.10966 & & & & & 0.00344051 & 0.00461046 & -0.0011257 & $4.4247 E-05$ & & & & & & & & & \\
\hline 28 & 27 & 0.11689 & 0.11427 & & & & & 0.00491413 & 0.00460386 & 0.00034792 & $3.7646 \mathrm{E}-05$ & & & & & & & & & \\
\hline 29 & 28 & 0.12181 & 0.11888 & & & & & 0.00306764 & 0.00455231 & -0.0014986 & $-1.39 \mathrm{E}-05$ & & & & & & & & & \\
\hline 30 & 29 & 0.12488 & 0.12343 & & & & & 0.00253909 & 0.00452978 & -0.0020271 & $.3 .643 \mathrm{E}-05$ & & & & & & & & & \\
\hline 31. & 30 & 0.12741 & $\begin{array}{l}0.12796 \\
\text { shifted corve }\end{array}$ & & & & & $\begin{array}{l}0.00505428 \\
\text { Signas noise }\end{array}$ & 0.00454993 & 0.00048807 & $-1.628 \mathrm{E}-05$ & & & & & & & & & \\
\hline
\end{tabular}

Fig. 6. Sheet "Signal noise check".

The second data quality metric (digital resolution, Sec. 2.1.3) is implemented in the sheet "Digital resolution check" (Fig. 7). Again, the sheet can handle up to 2,000 data points, but can be modified to handle larger numbers. Normalized data points are automatically copied from columns R and S of the sheet "Input data".

Information about the bins (count and percentages) is provided in columns $\mathrm{M}$ to $\mathrm{R}$, and the relative digital resolution is shown in cells F7 and F14 for $x$ and $y$ respectively. The relative digital resolution is calculated by dividing $z_{x}$ and $z_{y}$ (numbers corresponding to the bins having the highest percentages in columns $\mathrm{O}$ and $\mathrm{Q}$, excluding the zeroth bins) by the maximum acceptable value of 3 . If the values in cells F7 and F14 are lower than 1 (i.e., $z$ values less than 3 ), the cells are highlighted in green; otherwise, they are highlighted in red (indicating unacceptable digital resolution). 


\begin{tabular}{|c|c|c|c|c|c|c|c|c|c|c|c|c|c|c|c|c|c|c|c|c|}
\hline 4 & A & B & c & $E$ & $\mathrm{~F}$ & G & $\mathrm{H}$ & 1 & J & k & L & M & N & 0 & p & a & $\mathbf{R}$ & $s$ & $T$ & $u$ \\
\hline 1 & $\mathrm{i}$ & $x_{\text {porem }}$ & $Y_{\text {notem }}$ & $N=$ & 220 & & $\Delta x_{i}$ & $\Delta \Delta x_{i}$ & $\Delta y_{i}$ & $\Delta \Delta y_{i}$ & & $\mathrm{i}$ & Bin & $x$-count & $\%$ & $y$-count & $\%$ & & & \\
\hline 2 & 1 & 0 & 0 & & & & 8.3E- -05 & 0.00038 & 8E-06 & 5E-05 & & 0 & -0.00012 & 14 & $6.4 \%$ & 198 & $90.8 \%$ & & & \\
\hline 3 & 2 & 8.3E-05 & $8 E-06$ & $8=$ & 0.00024 & & -0.00029 & 0.00176 & $5.8 E-05$ & 0.00075 & & 1 & 0.00012 & 26 & $11.9 \%$ & 10 & $4.6 \%$ & & & \\
\hline 4 & 3 & -0.00021 & $6.6 E-05$ & & & & 0.00146 & 0.00049 & 0.0008 & 0.00149 & & 2 & 0.00037 & 34 & $15.6 \%$ & 2 & $0.9 \%$ & & & \\
\hline 5 & 4 & 0.00125 & 0.00087 & $\operatorname{Max} \times \%=$ & $15.6 \%$ & & 0.00097 & 0.00233 & 0.00229 & 0.00142 & & 3 & 0.00061 & 20 & $9.2 \%$ & 2 & $0.9 \%$ & & & \\
\hline 6 & 5 & 0.00223 & 0.00316 & $z_{x}=$ & 2 & & 0.0033 & 0.00297 & 0.00371 & 0.00115 & & 4 & 0.00085 & 30 & $13.8 \%$ & 1 & $0.5 \%$ & & & \\
\hline 7 & 6 & 0.00553 & 0.00688 & Rel. $x$ resolution $=$ & 0.67 & & 0.00627 & 0.00188 & 0.00486 & 0.00097 & & 5 & 0.0011 & 13 & $6.0 \%$ & 1 & $0.5 \%$ & & & \\
\hline 8 & 7 & 0.0118 & 0.01174 & & & & 0.00815 & 0.00224 & 0.00583 & 0.00063 & & 6 & 0.00134 & 16 & $7.3 \%$ & 2 & $0.9 \%$ & & & \\
\hline 9 & 8 & 0.01994 & 0.01757 & $\mathrm{X}$ digital resolut & ttion sufficien & & 0.00591 & 0.00108 & 0.00646 & 0.00012 & & 7 & 0.00159 & 17 & $7.8 \%$ & 0 & $0.0 \%$ & & & \\
\hline 10 & 9 & 0.02585 & 0.02403 & & & & 0.00699 & 0.00109 & 0.00658 & 0.00033 & & 8 & 0.00183 & 9 & $4.1 \%$ & 0 & $0.0 \%$ & & & \\
\hline 11 & 10 & 0.03284 & 0.03062 & & & & 0.00808 & 0.00032 & 0.00625 & 0.00045 & & 9 & 0.00208 & 11 & $5.0 \%$ & 0 & $0.0 \%$ & & & \\
\hline 12 & 11 & 0.04091 & 0.03687 & $\operatorname{Max} y \%=$ & $4.6 \%$ & & 0.0084 & 0.00347 & 0.0058 & 0.00039 & & 10 & 0.00232 & 10 & $4.6 \%$ & 0 & $0.0 \%$ & & & \\
\hline 13 & 12 & 0.04931 & 0.04267 & $z_{y}=$ & 1 & & 0.00493 & 0.00215 & 0.00541 & 0.00033 & & 11 & 0.00256 & 6 & $2.8 \%$ & 0 & $0.0 \%$ & & & \\
\hline 14 & 13 & 0.05424 & 0.04808 & Rel. y resolution = & 0.33 & & 0.00708 & 0.00052 & 0.00508 & 0.00017 & & 12 & 0.00281 & 2 & $0.9 \%$ & 0 & $0.0 \%$ & & & \\
\hline 15 & 14 & 0.06133 & 0.05316 & & & & 0.0076 & 0.00194 & 0.00491 & 2.5E-05 & & 13 & 0.00305 & 1 & $0.5 \%$ & 0 & $0.0 \%$ & & & \\
\hline 16 & 15 & 0.06893 & 0.05807 & $Y$ digital resolut & tion sufficien & & 0.00566 & $9.8 \mathrm{E}-05$ & 0.00489 & 0.00011 & & 14 & 0.0033 & 4 & $1.8 \%$ & 0 & $0.0 \%$ & & & \\
\hline 17 & 16 & 0.07459 & 0.06295 & & & & 0.00576 & 0.00259 & 0.00478 & 2.1E-05 & & 15 & 0.00354 & 1 & $0.5 \%$ & 0 & $0.0 \%$ & & & \\
\hline 18 & 17 & 0.08035 & 0.06773 & & & & 0.00317 & 0.00133 & 0.00476 & $1.5 \mathrm{E}-05$ & & 16 & 0.00378 & 2 & $0.9 \%$ & 0 & $0.0 \%$ & & & \\
\hline 19 & 18 & 0.08352 & 0.07249 & & & & 0.00184 & 0.00178 & 0.00477 & $5.1 \mathrm{E}-05$ & & 17 & 0.00403 & 0 & $0.0 \%$ & 2 & $0.9 \%$ & & & \\
\hline $20 \mid$ & 19 & 0.08535 & 0.07726 & & & & 0.00362 & 0.00013 & 0.00472 & 4.1E- 05 & & 18 & 0.00427 & 0 & $0.0 \%$ & 0 & $0.0 \%$ & & & \\
\hline 21 & 20 & 0.08897 & 0.08198 & & & & 0.00349 & 0.00109 & 0.00468 & $8.2 E-05$ & & 19 & 0.00452 & 1 & $0.5 \%$ & 0 & $0.0 \%$ & & & \\
\hline 22 & 21 & 0.09246 & 0.08666 & & & & 0.00458 & 0.00029 & 0.0046 & 2.1E-05 & & 20 & 0.00476 & 0 & $0.0 \%$ & 0 & $0.0 \%$ & & & \\
\hline 23 & 22 & 0.09704 & 0.09126 & & & & 0.00428 & 0.0003 & 0.00458 & $3 E-05$ & & 21 & 0.005 & 1 & $0.5 \%$ & 0 & $0.0 \%$ & & & \\
\hline 24 & 23 & 0.10132 & 0.09583 & & & & 0.00459 & 0.00036 & 0.00461 & 1.1E-05 & & 22 & 0.00525 & 0 & $0.0 \%$ & 0 & $0.0 \%$ & & & \\
\hline 25 & 24 & 0.10591 & 0.10044 & & & & 0.00422 & 0.0009 & 0.00462 & $1.1 \mathrm{E}-05$ & & 23 & 0.00549 & 0 & $0.0 \%$ & 0 & $0.0 \%$ & & & \\
\hline 26 & 25 & 0.11013 & 0.10506 & & & & 0.00332 & 0.00012 & 0.00461 & $3.9 \mathrm{E}-06$ & & 24 & 0.00574 & 0 & $0.0 \%$ & 0 & $0.0 \%$ & & & \\
\hline 27 & 26 & 0.11345 & 0.10966 & & & & 0.00344 & 0.00147 & 0.00461 & 6.6E-06 & & 25 & 0.00598 & 0 & $0.0 \%$ & 0 & $0.0 \%$ & & & \\
\hline 28 & 27 & 0.11689 & 0.11427 & & & & 0.00491 & 0.00185 & 0.0046 & $5.2 E-05$ & & 26 & 0.00623 & 0 & $0.0 \%$ & 0 & $0.0 \%$ & & & \\
\hline 29 & 28 & 0.12181 & 0.11888 & & & & 0.00307 & 0.00053 & 0.00455 & $2.3 E-05$ & & 27 & 0.00647 & 0 & $0.0 \%$ & 0 & $0.0 \%$ & & & \\
\hline 30 & 29 & 0.12488 & 0.12343 & & & & 0.00254 & 0.00252 & 0.00453 & $2 E-05$ & & 28 & 0.00671 & 0 & $0.0 \%$ & 0 & $0.0 \%$ & & & \\
\hline
\end{tabular}

Fig. 7. Sheet "Digital resolution check".

The actual linear regression is performed in the next sheet ("Regression”). The user needs to click "START REGRESSION", and the following results are displayed in column G (Fig. 8):

- Index of the first point of the optimum window (Start point)

- Index of the last point of the optimum window (End point)

- Minimum normalized residual

- Fitting line slope (for the shifted and normalized data set)

- Fitting line intercept (for the shifted and normalized data set).

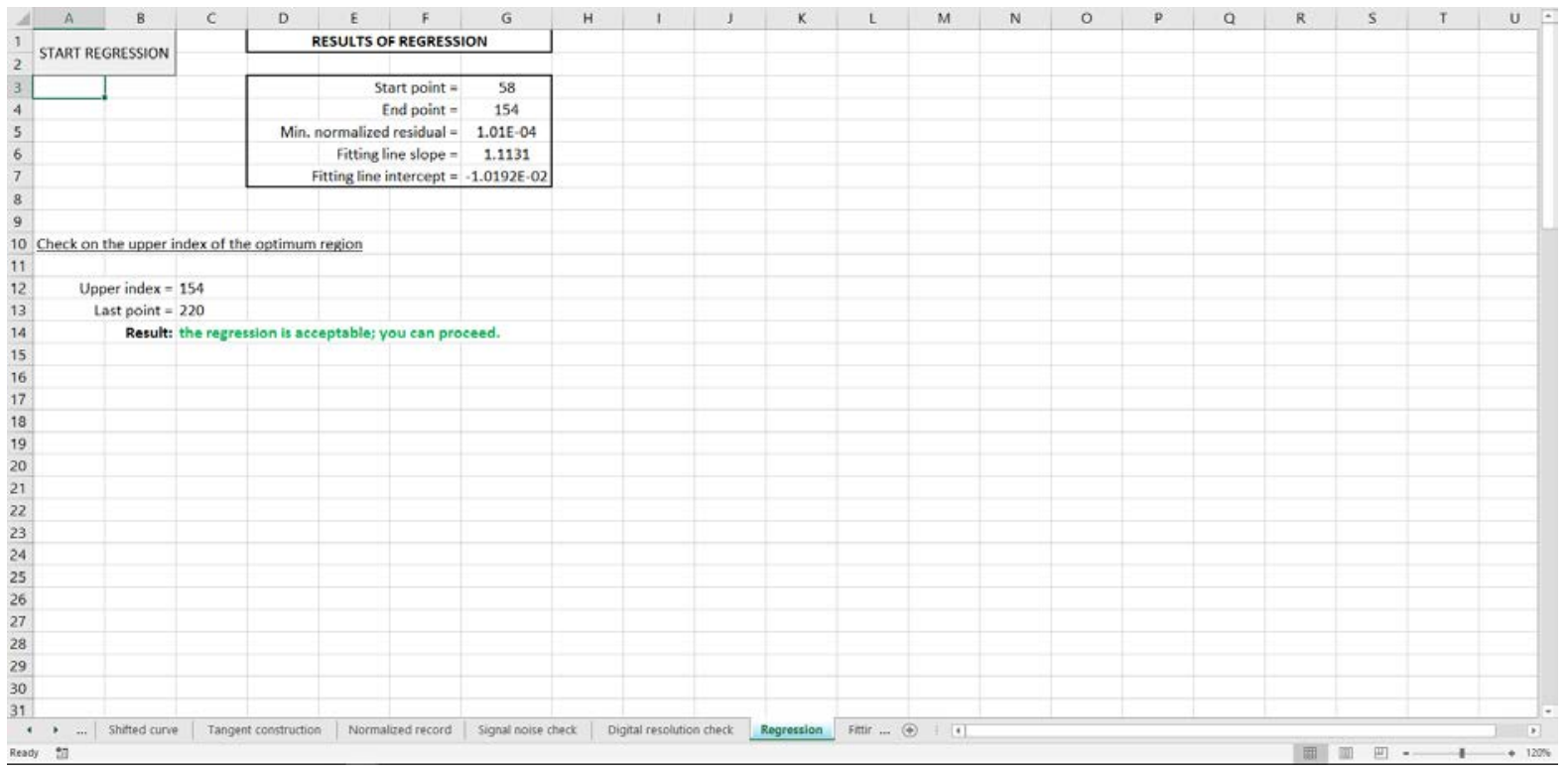

Fig. 8. Sheet "Regression". 
The lower part of the sheet "Regression" verifies if the last point of the fitted range coincides with the tangency point (Sec. 2.1.4). If it doesn't, the message "the regression is acceptable; you can proceed." is printed in bold green; if it does, the message "go back to 'Input data' and raise the offset point by $5 \%$ of max(y)" is printed in bold red.

The following sheet ("Fitting results") provides the un-normalized results of the linear regression (Sec. 2.1.7): final slope in cell D8, final intercept in cell D9, and true (i.e., un-normalized) intercept in cell D10. The remainder of this sheet contains information useful for plotting the final results, as will be addressed later. Note that the length of columns K to $M$ has to be adjusted based on the actual size of the data set (number of data points). A red balloon has been added to remind the user (Fig. 9).

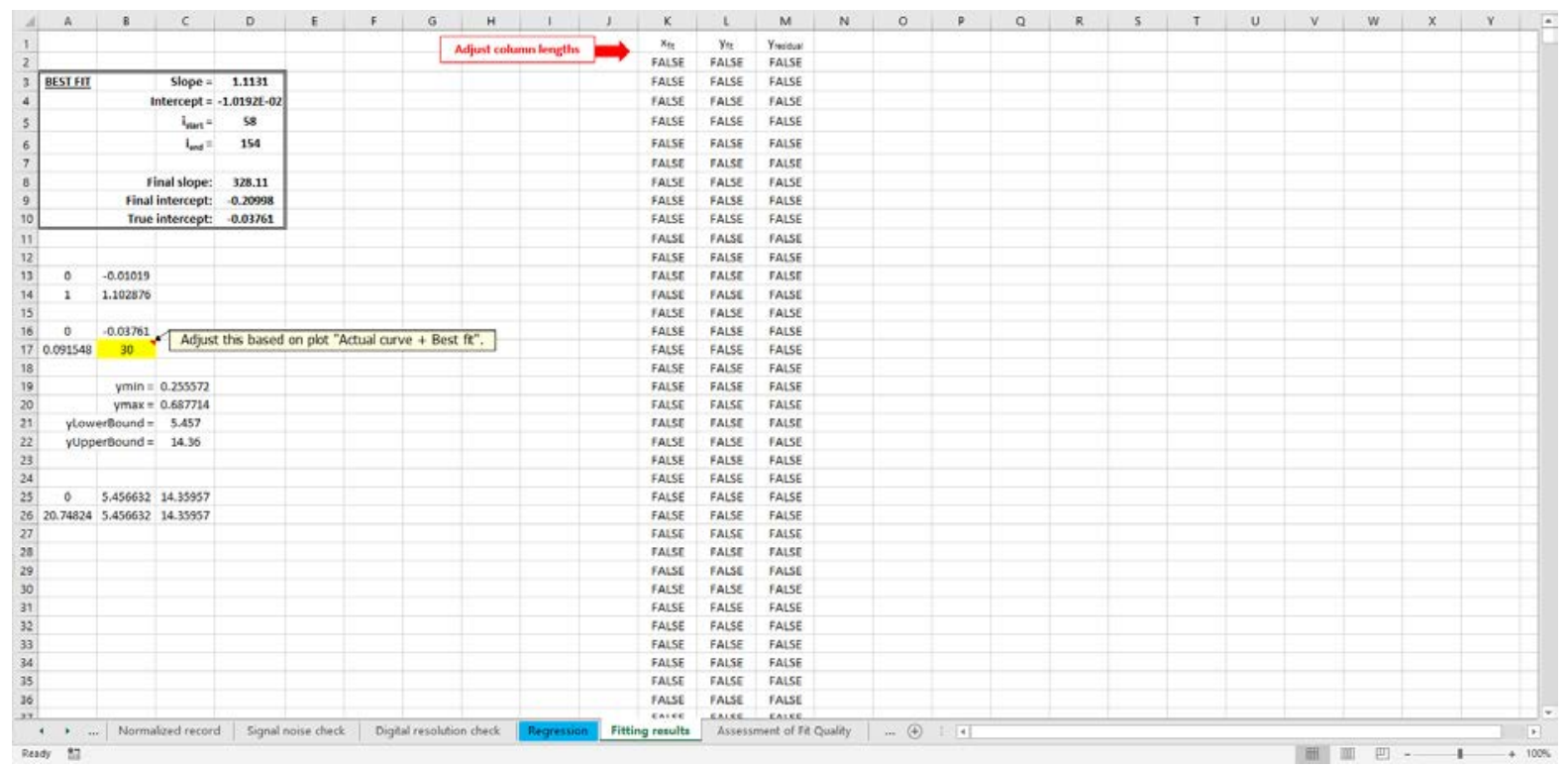

Fig. 9. Sheet "Fitting results".

Both fit quality metrics are analyzed in the sheet "Assessment of fit quality" (Sec. 2.1.5-2.1.6 and Fig. 10). Normalized $x$-values and $y$ residuals are displayed in columns $H$ and $\mathrm{I}$, while columns $\mathrm{J}$ and $\mathrm{K}$ provide the values contained in the first and fourth quartiles. Note that the user must adjust the length of columns $\mathrm{H}$ to $\mathrm{K}$ based on the number of data points (another red balloon provides a reminder). The normalized $y$ residuals are plotted as a function of normalized $x$ on the right side of the sheet.

The validity checks performed are:

- the number of points in the first and fourth quartiles (cells C13 and C19, respectively): acceptable if $\leq 5$;

- the relative residual slopes in the first and fourth quartiles (cells C16 and C22, respectively), calculated by dividing the actual slopes by the maximum allowed value ( $5 \%$ of the normalized slope): acceptable if $\leq 1$;

- relative fit range (cell E28), calculated by dividing 0.4 by the actual $y$-range: acceptable if $\leq 1$.

As usual, acceptable results are highlighted in green and unacceptable results are highlighted in red. 


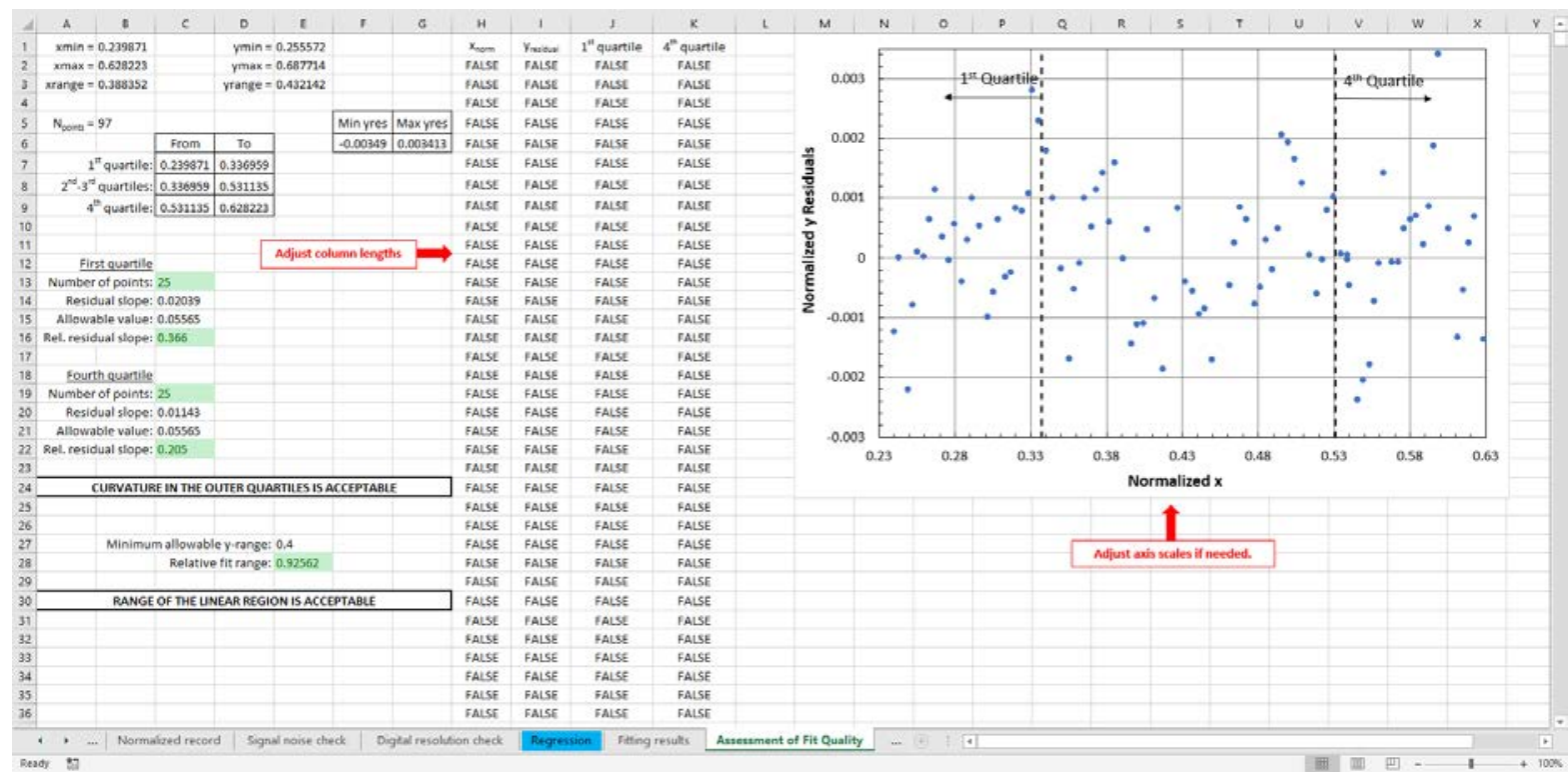

Fig. 10. Sheet "Assessment of Fit Quality".

The actual (i.e., un-normalized and with no offset) curve is displayed in the following sheet ("Actual curve + Best Fit"), with the calculated fitting line.

A summary of the results obtained, based on the "Report" section of ASTM E3076-18, is presented in the last sheet ("Results of Analysis”). Results corresponding to validity criteria are highlighted in green (acceptable) or red (unacceptable). The user can print a copy of the results on the default system printer by clicking "PRINT RESULTS" (Fig. 11).

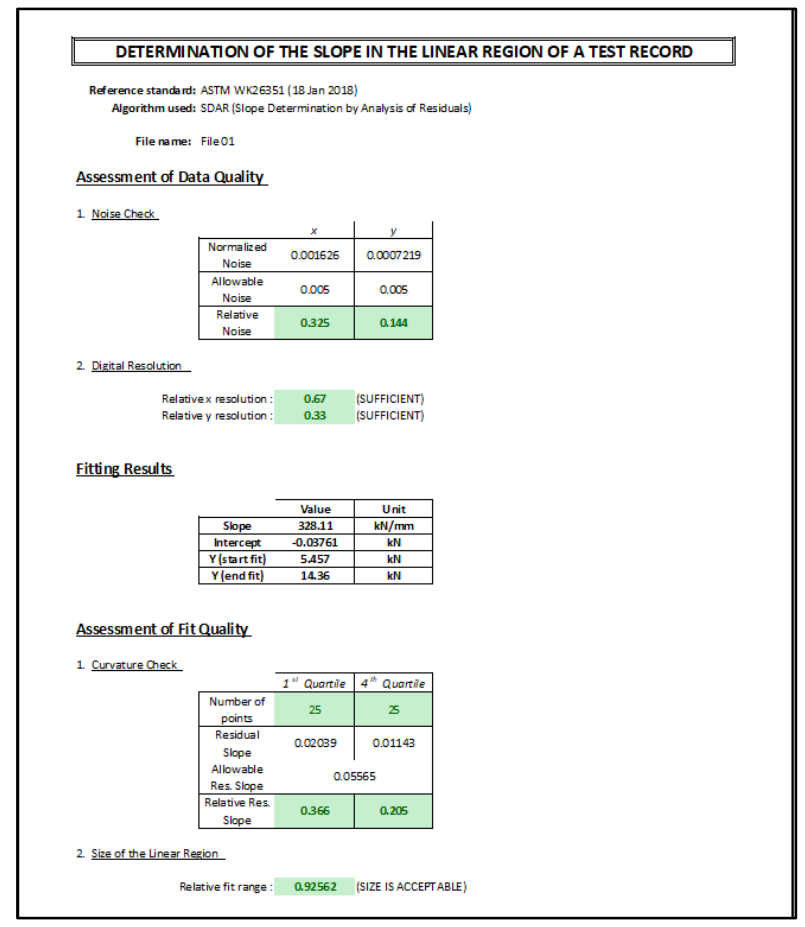

Fig. 11. Results printout. 


\subsection{Spreadsheet validation}

\subsubsection{ASTM E3076 example tensile data set: File 01}

In ASTM E3076-18, the demonstration of the SDAR algorithm is assisted by means of an example tensile test record. This file (File 01) has recently been made available from ASTM. The original curve is shown in Fig. 12.

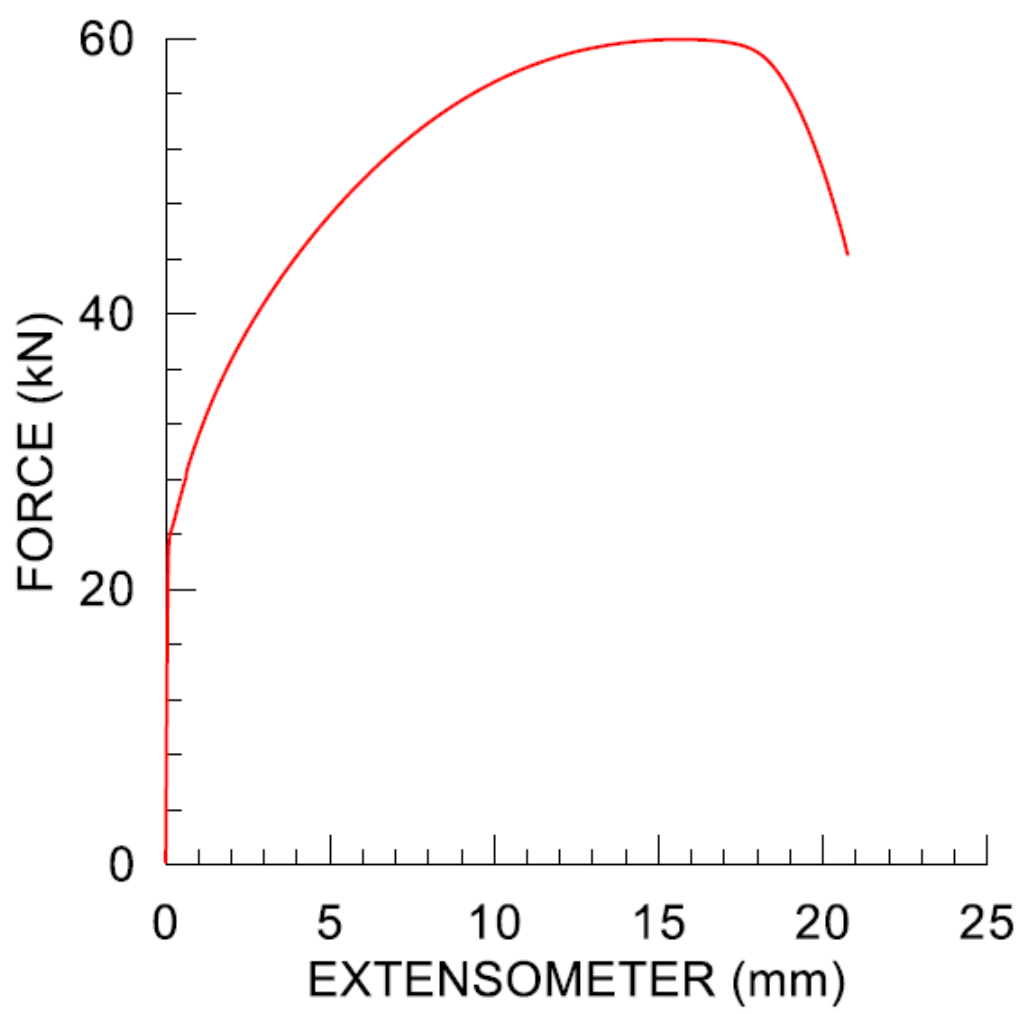

Fig. 12. Plot of the original test record File 01 (from ASTM E3076-18).

The Excel spreadsheet was tested on File 01, and the results of the analysis were compared with the values provided in ASTM E3076-18 (Table 1).

As shown by Table 1, the results of the two analyses are practically identical. The few small discrepancies (highlighted in bold red in the Table) are most likely attributable to rounding errors, which manifested themselves in a slightly different number of data points being fitted (see columns labeled "Lower OR index" and "Upper OR index" in Table 2).

This first validation can be therefore considered successful. 
Table 1. Comparison between ASTM E3076-18 and Excel spreadsheet for File 01.

\begin{tabular}{|c|c|c|c|}
\hline Parameter/result & ASTM E3076-18 & Excel spreadsheet & Difference \\
\hline xshift & 5.792E-5 & 5.792E-5 & $0 \%$ \\
\hline yshift & 0.1914 & 0.01914 & $0 \%$ \\
\hline$x_{1}$ & 0.01016 & 0.01016 & $0 \%$ \\
\hline$y_{1}$ & 3.012 & 3.012 & $0 \%$ \\
\hline yoffset (offset: $15 \%$ ) & 11.98 & 12.01 & $-0.2 \%$ \\
\hline Tangency pt index & 220 & 220 & 0 \\
\hline xtangent & 0.06989 & 0.06989 & $0 \%$ \\
\hline ytangent & 20.60 & 20.60 & $0 \%$ \\
\hline Relative noise $(x)$ & 0.325 & 0.325 & $0 \%$ \\
\hline Relative noise (y) & 0.144 & 0.144 & $0 \%$ \\
\hline$z$ ( $x$ variable) & 2 & 2 & 0 \\
\hline Relative $x$ resolution & 0.67 & 0.67 & $0 \%$ \\
\hline$z$ (y variable) & 1 & 1 & 0 \\
\hline Relative $y$ resolution & 0.33 & 0.33 & $0 \%$ \\
\hline Index start fit range & 58 & 58 & 0 \\
\hline Index end fit range & 154 & 154 & 0 \\
\hline Slope $m$ & 1.1131 & 1.1131 & $0 \%$ \\
\hline Intercept & $-1.0192 \mathrm{E}-2$ & $-1.0192 \mathrm{E}-2$ & $0 \%$ \\
\hline Relative residual slope $(x)$ & 0.366 & 0.366 & $0 \%$ \\
\hline Relative residual slope $(y)$ & 0.205 & 0.205 & $0 \%$ \\
\hline Relative fit range & 0.92565 & 0.92562 & $-0.0001 \%$ \\
\hline Final slope & 328.10 & 328.11 & $-0.003 \%$ \\
\hline Final intercept & -0.20998 & -0.20998 & $0 \%$ \\
\hline True intercept & -0.03761 & -0.03761 & $0 \%$ \\
\hline Lower bound $y$-range & 5.457 & 5.457 & $0 \%$ \\
\hline Upper bound $y$-range & 14.36 & 14.36 & $0 \%$ \\
\hline
\end{tabular}

\subsubsection{ASTM E08.03 analytical round-robin for the validation of the SDAR algorithm}

While developing ASTM E3076, the ASTM E08.03 sub-committee ran an analytical roundrobin, wherein eight "synthetic" data sets (created artificially by modifying an initial "perfect" data set) had to be analyzed in accordance with the then-current draft standard practice.

Through communication with the ASTM sub-committee chairman, S. Graham [9], the author obtained the nine data sets, which were defined/classified as follows:

- SDAR-rr1: perfect data set with a small amount of noise added to the baseline; 
- SDAR-rr2: increase initial curvature;

- SDAR-rr3: increase sampling rate (from $10 \mathrm{~Hz}$ to $20 \mathrm{~Hz}$ );

- SDAR-rr4: adjust pt. A to reduce fit range;

- SDAR-rr5: SDAR-rr1 with Y-noise;

- SDAR-rr6: SDAR-rr1 with low Y-resolution;

- SDAR-rr7: noise in $\mathrm{X}$ and gradual slope change;

- SDAR-rr8: low X-resolution.

S. Graham also provided the complete results for each of the eight data sets, including validity checks.

The eight data sets were analyzed by means of the Excel spreadsheet previously described. The comparison between our results and the round-robin outcome (average of participants' results) is illustrated in Table 2 (regression results) and Table 3 (digital resolution, noise, slopes in tails, and relative fit range).

Table 2. Comparison between SDAR round-robin (ASTM r-r) and Excel (NIST) analysis results (Part 1: regression results). Relative differences greater than $2 \%$ are highlighted in bold red.

\begin{tabular}{|c|c|c|c|c|c|c|c|c|c|c|c|c|c|c|}
\hline \multirow[b]{2}{*}{$\begin{array}{l}\text { Data } \\
\text { Set }\end{array}$} & \multirow[b]{2}{*}{ Analysis } & \multicolumn{13}{|c|}{ REGRESSION } \\
\hline & & $\begin{array}{l}\text { Final } \\
\text { Slope }\end{array}$ & $\begin{array}{c}\text { True } \\
\text { Intercept }\end{array}$ & $\begin{array}{c}\text { Lower } \\
\text { y bound }\end{array}$ & $\begin{array}{l}\text { Upper } \\
\text { y bound }\end{array}$ & $\begin{array}{c}\text { Lower } \\
\text { OR index }\end{array}$ & $\begin{array}{c}\text { Upper } \\
\text { OR index }\end{array}$ & $\begin{array}{c}\text { Lowest } \\
\text { norm.res. }\end{array}$ & $\begin{array}{c}\text { OR } \\
\text { Slope }\end{array}$ & $\begin{array}{c}\text { OR } \\
\text { Intercept }\end{array}$ & $\begin{array}{l}\text { OR } \\
x_{\text {min }}\end{array}$ & $\begin{array}{c}\text { OR } \\
x_{\max }\end{array}$ & $\begin{array}{l}\text { OR } \\
y_{\text {min }}\end{array}$ & $\begin{array}{c}\text { OR } \\
y_{\max }\end{array}$ \\
\hline \multirow{3}{*}{ SDARrr-1 } & $r-r$ & 56267.3 & -37.692 & 63.776 & 300.366 & 17 & 61 & $3.88 \mathrm{E}-05$ & 1.1368 & -0.11539 & 0.2589 & 0.9256 & 0.1805 & 0.9352 \\
\hline & NIST & 56264.6 & -37.433 & 58.942 & 305.200 & 18 & 62 & $3.88 \mathrm{E}-05$ & 1.1368 & -0.11539 & 0.2589 & 0.9256 & 0.1805 & 0.9352 \\
\hline & $\Delta$ & $-0.005 \%$ & 0.259 & -4.83 & 4.83 & 1 & 1 & $0.001 \%$ & $0.002 \%$ & $0.003 \%$ & $0.010 \%$ & $0.004 \%$ & $-0.016 \%$ & $-0.002 \%$ \\
\hline \multirow{3}{*}{ SDARrr-2 } & ASTM r-r & 120.911 & 0.22407 & 2.808 & 43.784 & 197 & 340 & $8.65 \mathrm{E}-05$ & 1.0331 & 0.004536 & 0.2746 & 0.755 & 0.2883 & 0.783 \\
\hline & NIST & 120.835 & 1.812 & 16.981 & 43.305 & 198 & 341 & $8.65 \mathrm{E}-05$ & 1.0331 & 0.004536 & 0.2746 & 0.7550 & 0.2883 & 0.7830 \\
\hline & $\Delta$ & $-0.063 \%$ & 1.588 & 14.17 & -0.48 & 1 & 1 & $0.001 \%$ & $0.001 \%$ & $-0.001 \%$ & $-0.011 \%$ & $-0.001 \%$ & $-0.007 \%$ & $0.001 \%$ \\
\hline \multirow{3}{*}{ SDARrr-3 } & ASTM r-r & 128.603 & 1.3507 & 7.363 & 55.814 & 261 & 1096 & $3.00 \mathrm{E}-04$ & 1.0075 & 0.02122 & 0.1009 & 0.7545 & 0.1202 & 0.7756 \\
\hline & NIST & 128.472 & 4.011 & 10.306 & 52.614 & 262 & 1097 & 3.00E-04 & 1.0075 & 0.02122 & 0.1009 & 0.7545 & 0.1202 & 0.7756 \\
\hline & $\Delta$ & $-0.102 \%$ & 2.661 & 2.94 & -3.20 & 1 & 1 & $0.001 \%$ & $-0.005 \%$ & $0.001 \%$ & $-0.005 \%$ & $-0.001 \%$ & $-0.036 \%$ & $0.002 \%$ \\
\hline \multirow{3}{*}{ SDARrr-4 } & ASTM r-r & 97339.3 & -285.27 & 196.045 & 317.639 & 146 & 186 & $4.60 \mathrm{E}-04$ & 1.8878 & -0.84683 & 0.7461 & 0.9472 & 0.5663 & 0.9433 \\
\hline & NIST & 97660.3 & -286.79 & 196.045 & 317.639 & 149 & 187 & 4.31E-04 & 1.8949 & -0.85306 & 0.7559 & 0.9472 & 0.5821 & 0.9433 \\
\hline & $\Delta$ & $0.330 \%$ & -1.516 & 0.00 & 0.00 & 3 & 1 & $-6.337 \%$ & $0.375 \%$ & $0.736 \%$ & $1.310 \%$ & $-0.004 \%$ & $2.784 \%$ & $-0.002 \%$ \\
\hline \multirow{3}{*}{ SDARrr-5 } & ASTM r-r & 55537.7 & -32.189 & 29.938 & 329.865 & 10 & 66 & $1.20 \mathrm{E}-03$ & 1.1038 & -0.09733 & 0.1503 & 1 & 0.083 & 1 \\
\hline & NIST & 55486.8 & -34.31 & 25.763 & 329.865 & 11 & 67 & $1.20 \mathrm{E}-03$ & 1.1205 & -0.09464 & 0.1439 & 0.957749 & 0.081 & 0.972298 \\
\hline & $\Delta$ & $-0.092 \%$ & -2.117 & -4.18 & 0.00 & 1 & 1 & $-0.002 \%$ & $1.515 \%$ & $-2.771 \%$ & $-4.251 \%$ & $-4.225 \%$ & $-2.784 \%$ & $-2.770 \%$ \\
\hline \multirow{3}{*}{ SDARrr- 6} & ASTM r-r & 15589.9 & 10.772 & 42.969 & 218.75 & 39 & 194 & $6.11 \mathrm{E}-04$ & 0.97382 & 0.04702 & 0.1839 & 0.915 & 0.2241 & 0.931 \\
\hline & NIST & 15611.2 & 10.653 & 50.781 & 210.938 & 40 & 195 & $6.11 \mathrm{E}-04$ & 0.97382 & 0.04702 & 0.1839 & 0.915 & 0.2241 & 0.931 \\
\hline & $\Delta$ & $0.137 \%$ & -0.119 & 7.81 & -7.81 & 1 & 1 & $0.000 \%$ & $0.000 \%$ & $0.000 \%$ & $0.022 \%$ & $0.001 \%$ & $0.017 \%$ & $0.004 \%$ \\
\hline \multirow{3}{*}{ SDARrr-7 } & ASTM r-r & 13812.3 & 33.064 & 60.608 & 238.586 & 131 & 855 & $1.31 \mathrm{E}-03$ & 0.89715 & 0.12875 & 0.1248 & 0.8512 & 0.2377 & 0.8863 \\
\hline & NIST & 13804.2 & 33.926 & 61.249 & 228.271 & 132 & 856 & $1.31 \mathrm{E}-03$ & 0.89715 & 0.128747 & 0.1224 & 0.859 & 0.2377 & 0.887 \\
\hline & $\Delta$ & $-0.059 \%$ & 0.862 & 0.64 & -10.31 & 1 & 1 & $0.001 \%$ & $0.000 \%$ & $-0.002 \%$ & $-1.907 \%$ & $0.923 \%$ & $0.012 \%$ & $0.039 \%$ \\
\hline \multirow{3}{*}{ SDARrr-8 } & ASTM r-r & 15722.8 & 13.804 & 44.556 & 198.944 & 32 & 180 & $7.02 \mathrm{E}-04$ & 0.92303 & 0.069182 & 0.1702 & 1 & 0.2169 & 1 \\
\hline & NIST & 15702.0 & 13.752 & 43.213 & 200.928 & 33 & 184 & $6.83 \mathrm{E}-04$ & 0.95109 & 0.068904 & 0.1633 & 0.980 & 0.2146 & 0.994 \\
\hline & $\Delta$ & $-0.132 \%$ & -0.004 & -1.34 & 1.98 & 1 & 4 & $-2.765 \%$ & $3.040 \%$ & $-0.401 \%$ & $-4.074 \%$ & $-2.041 \%$ & $-1.071 \%$ & $-0.558 \%$ \\
\hline
\end{tabular}


Table 3. Comparison between SDAR round-robin (ASTM r-r) and Excel (NIST) analysis results (Part 2: resolution, noise, tail slopes, and relative fit range). Acceptable results are highlighted in green, unacceptable in red.

\begin{tabular}{|c|c|c|c|c|c|c|c|c|c|c|c|c|c|c|}
\hline \multirow[b]{2}{*}{$\begin{array}{l}\text { Data } \\
\text { Set }\end{array}$} & \multirow[b]{2}{*}{ Analysis } & \multicolumn{6}{|c|}{ DIGITAL RESOLUTION } & \multicolumn{2}{|c|}{ Noise } & \multicolumn{4}{|c|}{ Slopes in Tails } & \multirow{2}{*}{$\begin{array}{c}\text { Relative } \\
\text { Fit } \\
\text { Range }\end{array}$} \\
\hline & & $\begin{array}{c}\text { Relative } \\
\text { X res }\end{array}$ & $\begin{array}{c}X \\
\% \max \end{array}$ & $\begin{array}{c}\mathrm{X} \\
\text { \% zero }\end{array}$ & $\begin{array}{c}\text { Relative } \\
\text { Y res }\end{array}$ & $\begin{array}{c}Y \\
\% \max \end{array}$ & $\begin{array}{c}Y \\
\text { \% zero }\end{array}$ & $\begin{array}{c}\text { Rel } \\
\text { X noise }\end{array}$ & $\begin{array}{c}\text { Rel } \\
\text { Y noise }\end{array}$ & $\begin{array}{l}\text { Relative } \\
\text { Slope Q1 }\end{array}$ & $\begin{array}{c}\text { \# points } \\
\text { Q1 }\end{array}$ & $\begin{array}{l}\text { Relative } \\
\text { Slope Q4 }\end{array}$ & $\begin{array}{c}\text { \# points } \\
\text { Q4 }\end{array}$ & \\
\hline \multirow{3}{*}{ SDARrr-1 } & ASTM r-r & 0.33 & 21.5 & 1.5 & 1 & 24.6 & 0 & 0.251 & 0.285 & -0.276 & 11 & 0.177 & 11 & 1.813 \\
\hline & NIST & 0.33 & 21.5 & 1.5 & 1.0 & 24.6 & 0.0 & 0.214 & 0.694 & -0.269 & 11 & 0.034 & 12 & 1.887 \\
\hline & $\Delta$ & $0.00 \%$ & 0.0 & 0.0 & 0.0 & 0.0 & 0.0 & $-14.719 \%$ & $143.645 \%$ & $-2.547 \%$ & 0 & $-80.853 \%$ & 1 & $4.070 \%$ \\
\hline \multirow{3}{*}{ SDARrr-2 } & ASTM r-r & 1 & 14.6 & 6.4 & 5.67 & 6.4 & 2.3 & 0.266 & 0.275 & -1.204 & 133 & -0.358 & 46 & 1.925 \\
\hline & NIST & 1 & 14.6 & 6.4 & 5.67 & 6.4 & 2.3 & 0.340 & 0.482 & -0.144 & 47 & -0.483 & 29 & 1.237 \\
\hline & $\Delta$ & $0.00 \%$ & 0.0 & 0.0 & 0.0 & 0.0 & 0.0 & $27.780 \%$ & $75.148 \%$ & $-88.01 \%$ & -86 & $34.84 \%$ & -17 & $-35.750 \%$ \\
\hline \multirow{3}{*}{ SDARrr-3 } & ASTM r-r & 0.33 & 7.7 & 3.9 & 0.33 & 45.7 & 31.1 & 0.659 & 0.664 & 0.807 & 323 & -1.124 & 199 & 1.877 \\
\hline & NIST & 0.33 & 7.7 & 3.9 & 0.33 & 45.7 & 31.1 & 0.443 & 0.068 & -0.158 & 248 & -0.546 & 0 & 1.639 \\
\hline & $\Delta$ & $1.01 \%$ & 0.0 & 0.0 & 0.0 & 0.0 & 0.0 & $-32.731 \%$ & $-89.696 \%$ & $-119.59 \%$ & -75 & $-51.38 \%$ & -199 & $-12.699 \%$ \\
\hline \multirow{3}{*}{ SDARrr-4 } & ASTM r-r & 0.33 & 15.9 & 5.1 & 0.33 & 11.3 & 5.1 & 0.265 & 0.501 & -1.159 & 1.6 & -0.937 & 1.8 & 0.903 \\
\hline & NIST & 0.33 & 15.9 & 5.1 & 0.33 & 11.3 & 5.1 & 0.255 & 0.692 & -1.221 & 10 & -1.000 & 0 & 0.903 \\
\hline & $\Delta$ & $1.010 \%$ & 0.0 & 0.0 & 0.0 & 0.0 & 0.0 & $-3.953 \%$ & $38.154 \%$ & $5.35 \%$ & 8.4 & $6.68 \%$ & -1.8 & $0.006 \%$ \\
\hline \multirow{3}{*}{ SDARrr-5 } & ASTM r-r & 1.33 & 12.3 & 3.1 & 15.7 & 4.6 & 0 & 1.759 & 1.942 & -0.957 & 14 & -1.159 & 14 & 2.261 \\
\hline & NIST & 1.00 & 14.7 & 2.9 & 15.3 & 4.4 & 0 & 0.229 & 2.565 & -1.206 & 15 & -1.614 & 0 & 2.229 \\
\hline & $\Delta$ & $-24.812 \%$ & 2.4 & -0.2 & -0.4 & -0.2 & 0.0 & $-86.962 \%$ & $32.081 \%$ & $25.98 \%$ & 1 & $39.23 \%$ & -14 & $-1.414 \%$ \\
\hline \multirow{3}{*}{ SDARrr-6 } & ASTM r-r & 0.33 & 26.5 & 0 & 23.7 & 54.5 & 45.5 & 1.057 & 1.029 & 1.081 & 43 & -1.145 & 43 & 1.94 \\
\hline & NIST & 0.33 & 26.5 & 73.5 & 23.7 & 54.5 & 45.5 & 0.009 & 1.541 & 0.407 & 39 & -0.624 & 0 & 1.77 \\
\hline & $\Delta$ & $1.010 \%$ & 0.0 & 73.5 & 0.0 & 0.0 & 0.0 & $-99.113 \%$ & $49.745 \%$ & $-62.30 \%$ & -4 & $-45.47 \%$ & -43 & $-8.905 \%$ \\
\hline \multirow{3}{*}{ SDARrr-7 } & ASTM r-r & 13 & 2.07 & 0.5 & 0.33 & 11 & 0 & 1.512 & 1.357 & 1.486 & 192 & -2.385 & 216 & 1.729 \\
\hline & NIST & 13 & 2.1 & 0.5 & 0.33 & 11.0 & 9.6 & 1.989 & 0.221 & 0.894 & 176 & -1.522 & 0 & 1.622 \\
\hline & $\Delta$ & $0.000 \%$ & 0.0 & 0.0 & 0.0 & 0.0 & 9.6 & $31.565 \%$ & $-83.713 \%$ & $-39.82 \%$ & -16 & $-36.17 \%$ & -216 & $-6.171 \%$ \\
\hline \multirow{3}{*}{ SDARrr-8 } & ASTM r-r & 29 & 52.5 & 47.5 & 1.33 & 10.6 & 1.7 & 1.301 & 1.202 & 1.131 & 38 & -0.225 & 38 & 1.929 \\
\hline & NIST & 28 & 53.0 & 47.0 & 0.33 & 10.8 & 1.6 & 1.803 & 0.317 & 0.624 & 39 & -0.404 & 0 & 1.950 \\
\hline & $\Delta$ & $-3.448 \%$ & 0.5 & -0.5 & -1.0 & 0.2 & -0.1 & $38.571 \%$ & $-73.592 \%$ & $-44.80 \%$ & 1 & $79.49 \%$ & -38 & $1.068 \%$ \\
\hline
\end{tabular}

The comparison shown in Table 2 shows excellent overall agreement between our results and the outcome of the ASTM round-robin. Discrepancies are considerably more significant, however, for the information presented in Table 3. In a few instances, the two analyses produced contradictory results for the validity checks.

When interrogated about such discrepancies, S. Graham responded [9]:

"The discrepancies in noise and tails/slopes may be due to recent changes in the SDAR standard practice. The round robin was run using an earlier version. The most recent version has a different algorithm for noise extraction and uses the optimum region as the final fit. The previous version has a second fit based on a censored data set as the final fit."

In light of these statements and particularly of the results shown in Table 2, this second and more in-depth validation of the Excel spreadsheet can also be considered successful. 


\section{3. "Stripped-down" version of the SDAR algorithm for the measurement of elastic compliance: Compliance calculation.xIsm spreadsheet}

As already stated in Section 1, measuring the slope of an elastic unload/reload cycle is a problem often encountered in fracture toughness or fatigue crack growth testing, since the measured compliance (displacement over force) enables one to infer the crack size by means of analytical relationships.

The calculation of compliance by linearly fitting the most linear region of an unload/reload cycle was implemented in another macro-enabled Microsoft Excel spreadsheet (Compliance calculation.xlsm). This spreadsheet runs an abridged version of the SDAR algorithm, which does not include the following analytical steps:

- Offset and truncation of the data set (Sec. 2.1.1)

- Check of noise in the data (Sec. 2.1.2)

- Check of digital resolution (Sec. 2.1.3)

- Check of curvature in the vicinity of the fit range (Sec. 2.1.5).

However, the spreadsheet does execute the following analytical steps:

- Normalization of the data set (Sec. 2.1.1)

- Establishment of the most linear part of the data set (Sec. 2.1.4)

- Linear regression of the most linear part of the data set (Sec. 2.1.4)

- Assessment of the size of the linear region (Sec. 2.1.6)

- Un-normalization of the slope and intercept (Sec. 2.1.7).

A screenshot of the spreadsheet is given in Fig. 13.

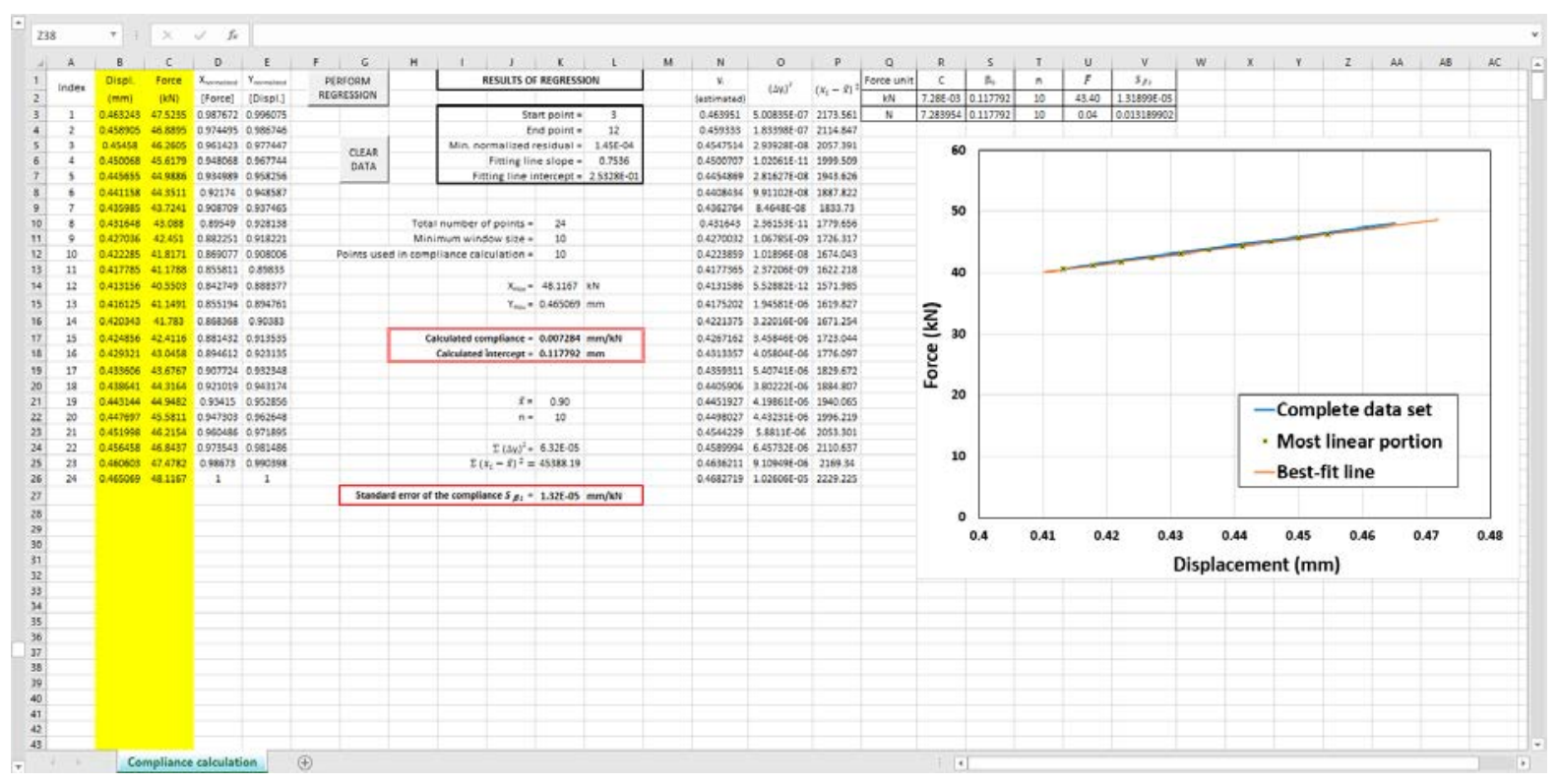

Fig. 13. Spreadsheet Compliance calculation.xlsm.

Data from the unload/reload cycle(s) are entered in columns B and C, which are highlighted in yellow. If data from a previous calculation are present, clicking "CLEAR 
DATA” erases them before pasting the new data. Columns A (data index), D (normalized force), and E (normalized displacement) ${ }^{2}$ are automatically populated.

Clicking “PERFORM REGRESSION” launches the SDAR algorithm, which first identifies the most linear region by searching for the minimum normalized residual, and then performs a linear fit of the most linear region. The results of the regression are provided in the central portion of the screen inside a box with thick black borders, namely:

- Index of the first point of the most linear region (Start point)

- Index of the last point of the most linear region (End point)

- Minimum normalized residual

- Slope of the fitting line

- Intercept of the fitting line.

Below the box, additional information is displayed, such as: total number of points in the data set, minimum window size (larger between 10 and $20 \%$ of the data set), number of points used in the compliance calculation, and maximum values of force and displacement $\left(X_{\max }\right.$ and $\left.Y_{\max }\right)$.

The calculated compliance and intercept are shown inside a box with double red borders. Below this, data needed for the assessment of the standard error in the calculated compliance ( $S_{\beta 1}$, see Sec. 3.2 below) are provided, and finally the calculated value of $S_{\beta 1}$ is displayed inside a box with thick red borders.

Columns $\mathrm{N}, \mathrm{O}$, and $\mathrm{P}$ contain intermediate calculations that are required for establishing the standard error of the compliance: $y_{i},\left(\Delta y_{i}\right)^{2},\left(x_{i}-\bar{x}\right)^{2}$.

The right top of the screen displays several calculated values, expressed in terms of different force units $(\mathrm{kN}$ or $\mathrm{N})$ : calculated compliance, $(C)$ calculated intercept $\left(\beta_{0}\right)$, number of points in the fitted region $(n)$, average force in the fitted region $(\bar{F})$, standard error of the compliance $\left(S_{\beta 1}\right)$.

Finally, the right side of the screen displays a plot of the force/displacement unload/reload cycle (blue line), with superimposed the data points representing the most linear region (" $x$ " symbols on yellow background) and the best-fit line (in orange). The plot has automatic scaling, but manual $\mathrm{X}$ and/or Y scales can be easily set for better visibility.

Typical calculation times (64-bit Windows 10 operating system ${ }^{3}$, x64-based Intel(R) Core(TM) i5-6440HQ CPU 2.60 GHz processor, 8 GB RAM) needed to obtain full results were found to be as follows:

- $\quad N=25: 0.2$ seconds

- $N=50: 0.3$ seconds

- $\quad N=100: 0.43$ seconds

- $\quad N=200: 1.56$ seconds

\footnotetext{
${ }^{2}$ Note that, in compliance calculation, force is the independent (X) variable and displacement is the dependent (Y) variable.

${ }^{3}$ Certain software is identified in this Technical Note to foster understanding. Such identification does not imply recommendation or endorsement by the National Institute of Standards and Technology, nor does it imply that the materials or equipment identified are necessarily the best available for the purpose.
} 
- $\quad N=500: 21.8$ seconds

- $\quad N=1000: 2.97$ minutes.

The calculation time is displayed in a pop-up window as soon as the regression is completed.

Currently, the spreadsheet can analyze up to 1000 data points, but it can be easily modified to accommodate a higher number of data points.

\section{1. “Regression” Visual Basic subroutine}

The implementation of the SDAR algorithm (in an abridged version) was accomplished through a Microsoft Visual Basic subroutine called "Regression", which is executed by clicking "PERFORM REGRESSION".

The Visual Basic source code of the subroutine can be found in Appendix 1, and could be easily translated in other commonly used programming languages (R, MATLAB, Mathcad, etc.).

\section{ASTM E1820-18: recommended best practices for calculating elastic compliance}

In the most recent revision of ASTM E1820 [3], Appendix X3 provides "recommended best practices for estimating crack size using elastic compliance". The most significant information provided in this Appendix (informative, not prescriptive) is summarized below.

To avoid non-linearities that are common at the beginning and end of an unload, it is recommended to discard the upper and lower $5 \%$ of the force range in the data set used for the compliance calculation.

It is also recommended that crack sizes be calculated from just the unloading part of the cycle; alternatively, it is permissible to use just the reload compliance or the average of the unload and reload compliances.

The standard error of the compliance, which is a measure of the uncertainty in the slope measurement and can be used to estimate the uncertainty in the critical toughness $J_{I c}$, is given by:

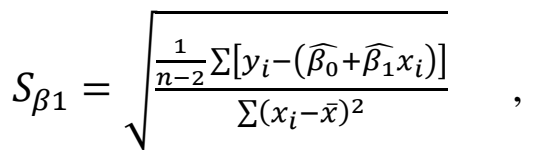

where $\widehat{\beta_{0}}$ and $\widehat{\beta_{1}}$ are estimates of intercept and compliance, respectively.

The uncertainty in $J_{I c}$ can be estimated from the root-mean-square (rms) of $S_{\beta 1}$, given by:

$$
e=\sqrt{\frac{\sum_{i=1}^{n}\left(s_{\beta 1}\right)_{i}^{2}}{n}},
$$

which must be non-dimensionalized using: 


$$
\tilde{e}=e \frac{E B_{e} W}{0.0254 \mathrm{~mm}} \quad,
$$

with: $E=$ Young's modulus, $B_{e}=$ effective specimen thickness, and $W=$ specimen width.

Based on the analysis of round-robin data [10], the uncertainty in $J_{\text {Ic }}$ due to noise in unload/reload data is less than $4 \%$ when the calculated $\tilde{e}$ is less than 400 [11].

\subsection{Use of the SDAR algorithm for compliance calculations on actual fracture toughness data sets}

\subsubsection{J-R curve tests on 1TC(T) specimens: round-robin organized by HZDR (Germany, 2012)}

In 2012, Hans-Werner Viehrig from the Helmholz Zentrum Dresden Rossendorf (HZDR, Germany) organized a $J$ - $R$ curve round-robin exercise on Compact Tension specimens of $25.4 \mathrm{~mm}$ thickness, 1TC(T), extracted from the reactor pressure vessel of Biblis Unit C, which was never put in service. ${ }^{4}$

NIST was invited to participate in the round-robin by testing three fatigue precracked and side-grooved 1TC(T) specimens at room temperature. The material was 22NiMoCr37 steel (equivalent to ASTM A533 Grade B), and specimens were extracted from the lower base metal ring, segment 220 AB-S, block UT2.

Tests were performed with the single-specimen elastic compliance technique, which allows obtaining a full $J-R$ curve from each specimen tested.

In the original analyses, elastic compliance was calculated by linearly fitting the force/load-line displacement data labeled as "Unload” or "Reload” by the MTS software used. ${ }^{5}$ Note that three successive unload/reload cycles were performed for each crack size measurement. For the original analyses conducted in 2012, only data between $20 \%$ of the minimum force and $80 \%$ of the maximum force were used in the linear regression.

The compliance calculations for the three specimens (BBL-CGW_23, BBL-CGW_24, BBL-CGW_25) were repeated using the spreadsheet "Compliance calculation.xls". The following approaches were included in the analyses (in order of computational complexity):

(a) Linear fitting of the first unload cycle only [U1].

(b) Linear fitting of the first reload cycle only [R1].

(c) Linear fitting of the first unload + reload cycle only [U1R1].

(d) Linear fitting of all three unload + reload cycles [UallRall].

(e) Mean value between the slope of the first unload cycle and the first reload cycle only [Mean U1R1].

For each specimen and every approach listed above, the outcome of the SDAR algorithm was compared with the results obtained by fitting all data in the selected cycle(s) and by fitting data in accordance with Appendix X3 of ASTM E1820 (only unloadings, excluding the upper and lower $5 \%$ of the force range in each cycle, see Sec. 3.2).

\footnotetext{
${ }^{4}$ After the decision was taken to shut down units A and B of the Biblis nuclear power plant in 2011, the Biblis vessel for the nevercommissioned unit $\mathrm{C}$ was scrapped and thereafter used for material testing.

${ }^{5}$ The MTS testing software labeled each data point as "Ramp”, "Extend Crack”, "Unload”, or "Reload”.
} 
The parameters used in the comparisons were:

- the measured $\left(a_{0}\right)$ and predicted $\left(a_{0 q}\right)^{6}$ initial crack size;

- the measured $\left(\Delta a_{p}\right)$ and predicted $\left(\Delta a_{p r e d}\right)$ total crack extension;

- the critical fracture toughness, $J_{Q}$ or $J_{I c}$.

Table 4 presents the overall comparisons for each specimen, each approach (U1, R1, U1R1, UallRall, and Mean U1R1) and each analysis method (fit all data, E1820 App. X3 recommended, and SDAR algorithm). For the initial crack size and the total crack extension, the prediction closest to the measured value is highlighted in green.

Table 4. Overall comparisons for the 1TC(T) specimens tested for the HZDR round-robin.

\begin{tabular}{|c|c|c|c|c|c|c|c|c|c|c|c|c|}
\hline \multirow{3}{*}{$\begin{array}{c}\text { Specimen } \\
\text { id }\end{array}$} & \multirow{3}{*}{$\begin{array}{c}\text { Approach } \\
\text { used }\end{array}$} & \multicolumn{4}{|c|}{ Initial crack size, $a_{0}(\mathrm{~mm})$} & \multicolumn{4}{|c|}{ Total crack extension, $\Delta a_{p}(\mathrm{~mm})$} & \multicolumn{3}{|c|}{$J_{Q} / J_{I c}(\mathrm{kN} / \mathrm{m})$} \\
\hline & & Measured & & $a_{0 q}$ & & Measured & & $\Delta \mathrm{a}_{\text {pred }}$ & & Fit & & SDAR \\
\hline & & & Fit all & E1820 & SDAR & & Fit all & E1820 & SDAR & all & recomm. & \\
\hline \multirow{5}{*}{ BBL-CGW_23 } & U1 & 25.07 & 25.37 & 25.40 & 25.28 & 3.87 & 3.50 & 3.47 & 3.53 & 780.37 & 795.54 & 572.23 \\
\hline & $\mathrm{R} 1$ & 25.07 & 25.02 & 25.02 & 24.82 & 3.87 & 3.75 & 3.71 & 3.84 & 701.20 & 714.63 & 570.86 \\
\hline & U1R1 & 25.07 & 24.92 & 24.91 & 24.78 & 3.87 & 3.65 & 3.63 & 3.81 & 771.99 & 759.59 & 605.30 \\
\hline & UallRall & 25.07 & 24.88 & 24.86 & 24.84 & 3.87 & 3.65 & 3.63 & 3.77 & 786.18 & 785.25 & 812.40 \\
\hline & Mean U1R1 & 25.07 & 25.20 & 25.18 & 25.05 & 3.87 & 3.63 & 3.62 & 3.54 & 775.91 & 789.00 & 603.28 \\
\hline \multirow{5}{*}{ BBL-CGW_24 } & U1 & 25.19 & 24.45 & 24.48 & 24.53 & 3.87 & 3.84 & 3.82 & 4.11 & 609.08 & 616.97 & 545.38 \\
\hline & R1 & 25.19 & 24.26 & 24.26 & 24.11 & 3.87 & 3.99 & 3.95 & 4.25 & 446.11 & 457.89 & 394.20 \\
\hline & U1R1 & 25.19 & 24.40 & 24.32 & 24.42 & 3.95 & 3.84 & 3.89 & 4.21 & 571.19 & 534.07 & 513.99 \\
\hline & UallRall & 25.19 & 24.28 & 24.31 & 24.09 & 3.95 & 3.94 & 3.88 & 4.14 & 527.14 & 561.73 & 401.63 \\
\hline & Mean U1R1 & 25.19 & 24.38 & 24.35 & 24.33 & 3.95 & 3.90 & 3.90 & 4.11 & 550.85 & 539.22 & 452.10 \\
\hline \multirow{5}{*}{ BBL-CGW_25 } & U1 & 25.39 & 24.67 & 24.68 & 24.56 & 3.69 & 3.23 & 3.21 & 3.55 & 805.84 & 807.01 & 676.55 \\
\hline & $\mathrm{R} 1$ & 25.39 & 24.48 & 24.46 & 24.21 & 3.69 & 3.48 & 3.46 & 3.95 & 729.61 & 729.92 & 629.57 \\
\hline & U1R1 & 25.39 & 24.56 & 24.55 & 24.47 & 3.69 & 3.34 & 3.32 & 3.64 & 775.08 & 788.49 & 679.79 \\
\hline & UallRall & 25.39 & 24.52 & 24.50 & 24.54 & 3.69 & 3.34 & 3.32 & 3.28 & 796.39 & 805.86 & 799.28 \\
\hline & Mean U1R1 & 25.39 & 24.58 & 24.57 & 24.39 & 3.69 & 3.35 & 3.34 & 3.75 & 771.37 & 767.93 & 701.76 \\
\hline
\end{tabular}

ASTM E1820-18 imposes the following validity requirements on the predicted initial crack size and total crack extension:

- The measured and the predicted values of initial crack size must not differ by more than the larger between $0.5 \mathrm{~mm}$ and $0.1 \%$ of the specimen width. In this case, the maximum allowed deviation is $0.508 \mathrm{~mm}$.

- The measured and the predicted values of total crack extension must not differ by more than the larger between $20 \%$ of the initial ligament size and $15 \%$ of the measured crack extension. In this case, the maximum allowed deviation is $0.58 \mathrm{~mm}$.

The absolute deviations for the tests examined are shown in Table 5, where valid data are highlighted in green and invalid data are highlighted in red.

\footnotetext{
${ }^{6}$ The predicted initial crack size is obtained, in accordance with Sec. A9.3.2 and Sec. A9.3.3. of ASTM E1820-18, by fitting all $J-\Delta a$ data points before maximum force.
} 
Table 5. Absolute differences for initial crack size and total crack extension.

\begin{tabular}{|c|c|c|c|c|c|c|c|}
\hline \multirow{2}{*}{$\begin{array}{l}\text { Specimen } \\
\text { id }\end{array}$} & \multirow{2}{*}{$\begin{array}{c}\text { Approach } \\
\text { used }\end{array}$} & \multicolumn{3}{|c|}{$\left|a_{0}-a_{0 q}\right|(m m)$} & \multicolumn{3}{|c|}{$\left|\Delta a_{p}-\Delta a_{\text {pred }}\right|(\mathrm{mm})$} \\
\hline & & Fit all & E1820 & SDAR & Fit all & E1820 & SDAR \\
\hline \multirow{5}{*}{ BBL-CGW_23 } & U1 & 0.30 & 0.33 & 0.21 & 0.36 & 0.39 & 0.34 \\
\hline & R1 & 0.05 & 0.06 & 0.25 & 0.12 & 0.15 & 0.03 \\
\hline & U1R1 & 0.15 & 0.16 & 0.29 & 0.22 & 0.24 & 0.06 \\
\hline & UallRall & 0.19 & 0.21 & 0.23 & 0.21 & 0.23 & 0.10 \\
\hline & Mean U1R1 & 0.13 & 0.11 & 0.02 & 0.24 & 0.25 & 0.33 \\
\hline \multirow{5}{*}{ BBL-CGW_24 } & $\mathrm{U} 1$ & 0.74 & 0.71 & 0.66 & 0.02 & 0.04 & 0.24 \\
\hline & R1 & 0.93 & 0.93 & 1.08 & 0.12 & 0.08 & 0.38 \\
\hline & U1R1 & 0.79 & 0.87 & 0.76 & 0.11 & 0.06 & 0.26 \\
\hline & UallRall & 0.91 & 0.88 & 1.10 & 0.01 & 0.07 & 0.19 \\
\hline & Mean U1R1 & 0.81 & 0.84 & 0.86 & 0.06 & 0.05 & 0.15 \\
\hline \multirow{5}{*}{ BBL-CGW_25 } & $\mathrm{U} 1$ & 0.71 & 0.70 & 0.83 & 0.46 & 0.47 & 0.13 \\
\hline & R1 & 0.90 & 0.93 & 1.18 & 0.21 & 0.23 & 0.27 \\
\hline & U1R1 & 1.22 & 1.24 & 1.34 & 0.18 & 0.19 & 0.18 \\
\hline & UallRall & 0.87 & 0.88 & 0.84 & 0.35 & 0.36 & 0.40 \\
\hline & Mean U1R1 & 0.81 & 0.82 & 1.00 & 0.33 & 0.35 & 0.06 \\
\hline
\end{tabular}

The relative spread of the calculated fracture toughness values is shown in Table 6 .

Table 6. Relative spread of the plane-strain toughness values $J_{Q} / J_{I c}$.

\begin{tabular}{|c|c|c|}
\hline $\begin{array}{c}\text { Specimen } \\
\text { id }\end{array}$ & $\begin{array}{c}\text { Approach } \\
\text { used }\end{array}$ & $\begin{array}{c}\text { Spread } \\
\text { in J } / \mathrm{J}_{\mathrm{I}}\end{array}$ \\
\hline & U1 & $31.2 \%$ \\
& R1 & $21.7 \%$ \\
BBL-CGW_23 & U1R1 & $23.4 \%$ \\
& UallRall & $3.4 \%$ \\
& Mean U1R1 & $25.7 \%$ \\
\hline & U1 & $12.1 \%$ \\
& R1 & $14.7 \%$ \\
BBL-CGW_24 & U1R1 & $10.6 \%$ \\
& UallRall & $32.2 \%$ \\
& Mean U1R1 & $19.2 \%$ \\
\hline & U1 & $17.1 \%$ \\
& R1 & $14.4 \%$ \\
BBL-CGW_25 & U1R1 & $33.0 \%$ \\
& UallRall & $1.2 \%$ \\
& Mean U1R1 & $9.3 \%$ \\
\hline
\end{tabular}

The results obtained are also illustrated in:

- Fig. 14 (comparison between $J_{Q}$ values obtained with the three different methods),

- Fig. 15 (comparison between measured and predicted initial crack sizes),

- Fig. 16 (comparison between measured and predicted crack extensions),

- Fig. 17 (differences between measured and predicted initial crack sizes),

- Fig. 18 (differences between measured and predicted crack extensions), and

- Fig. 19 (spread in $J_{Q}$ values obtained with the three different methods).

\subsubsection{Discussion}

Examination of Figs. 14 to 19 does not reveal clear trends or patterns. However, the following observations can be formulated. 
(a) Using the method recommended in Appendix X3 of ASTM E1820 as the reference approach, differences in terms of $J_{Q}$ are much more significant when the SDAR algorithm is used than when fitting all data (Fig. 14). Values obtained from SDAR tend to be consistently lower, and some of the differences are larger than $20 \%$ (which is the typical scatter for $J_{I C}$ values).

(b) For two of the three tests evaluated, predicted values of initial crack size $\left(a_{0 q}\right)$ are invalid with respect to the E1820 requirement $( \pm 0.01 \mathrm{~W})$, regardless of the approach used (Fig. 15). In general, SDAR appears to provide the lowest $a_{0 q}$ values.

(c) Conversely, all the predicted values of total crack extension satisfy the E1820 requirement $\left( \pm 0.15 \Delta a_{p}\right)$. In this case, the largest predicted values are obtained by means of the SDAR algorithm (Fig. 16).

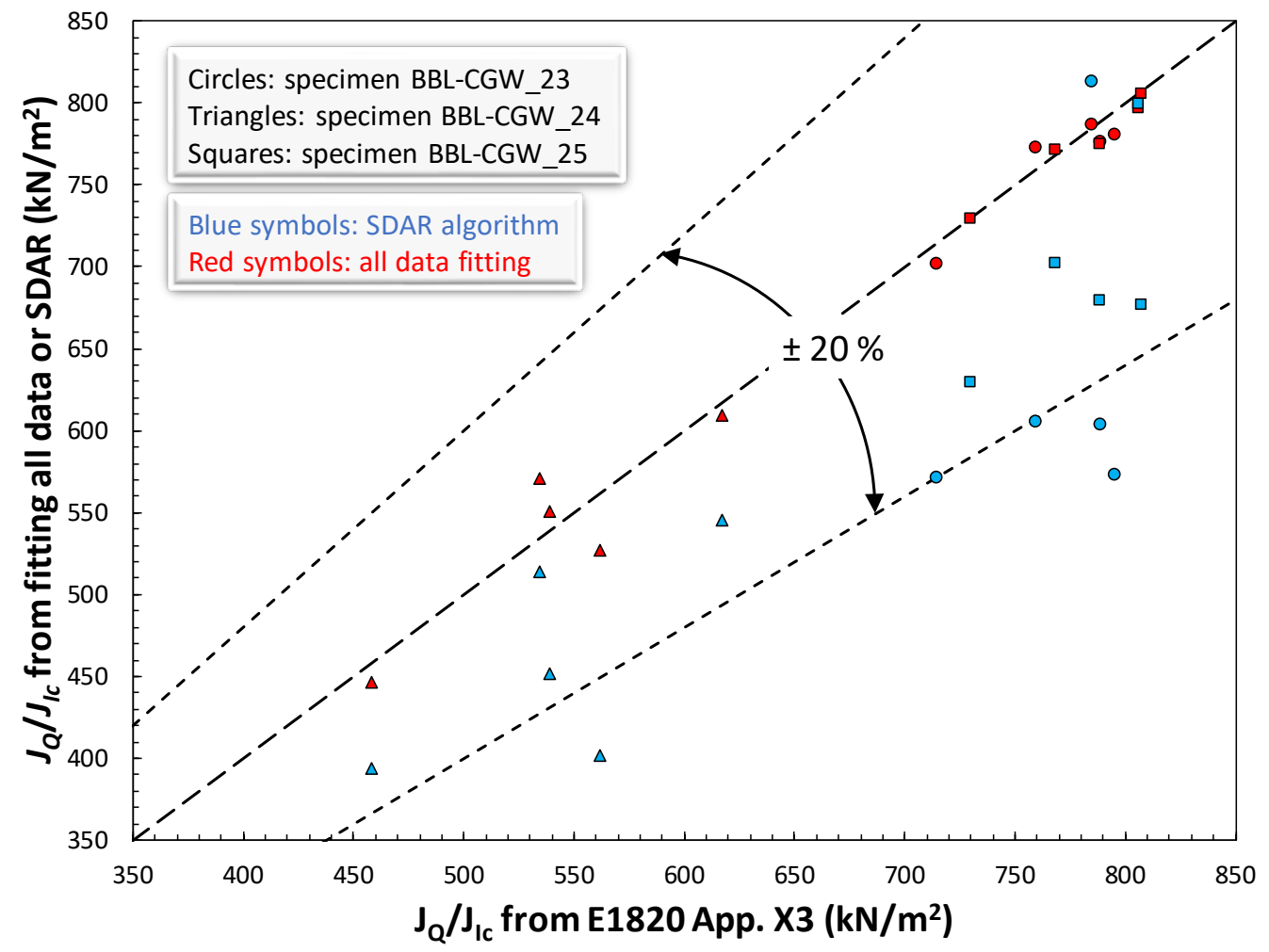

Fig. 14. Comparison between $J_{Q}$ calculated per ASTM E1820 App. X3, by fitting all data, and using the SDAR algorithm. $\pm 20 \%$ indicates the typical scatter band of $J_{I c}$ values. 


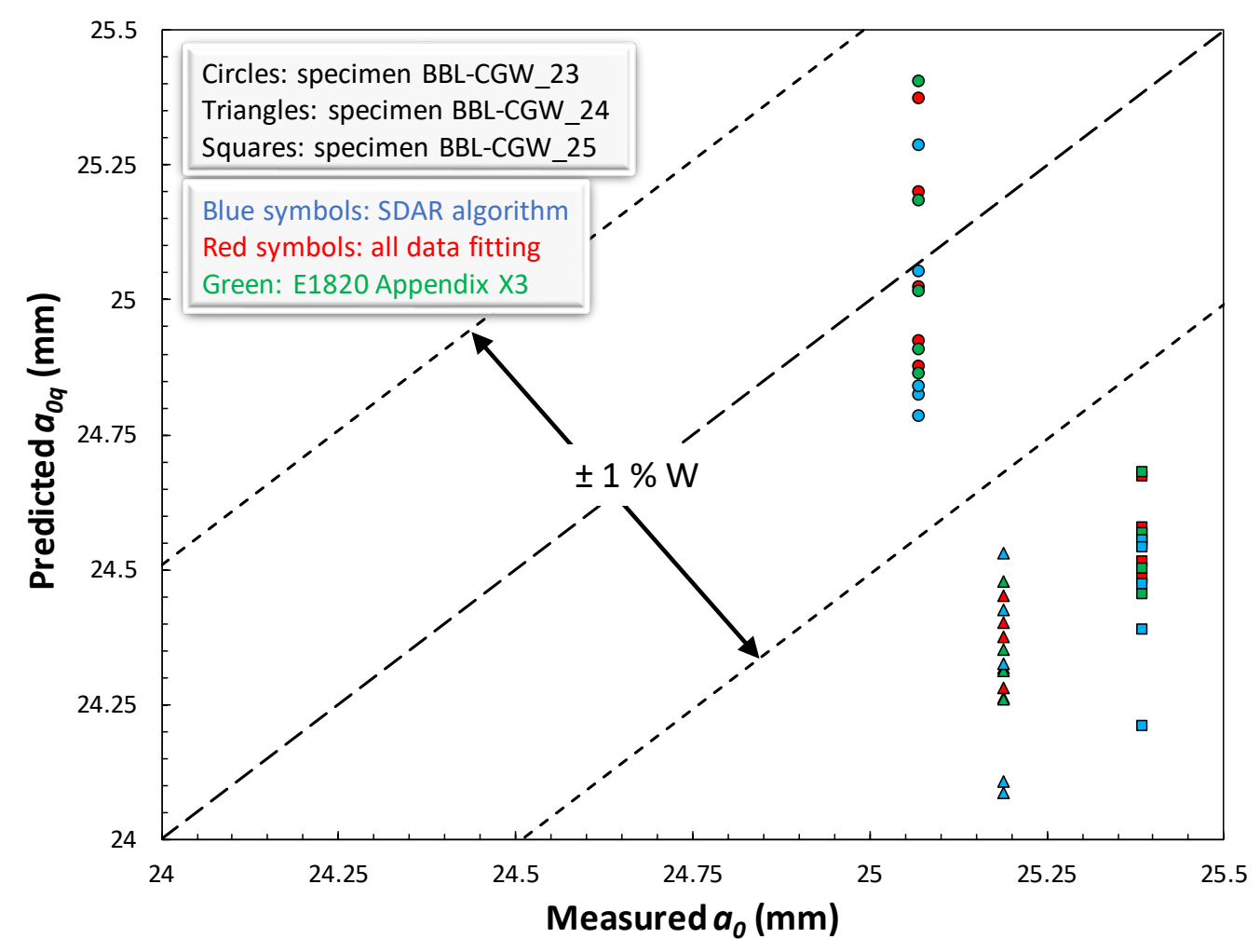

Fig. 15. Comparison between measured $a_{0}$ and $a_{0 q}$ calculated according to ASTM E1820 App. X3, by fitting all data, and by means of SDAR. $\pm 1 \% \mathrm{~W}$ is the ASTM requirement.

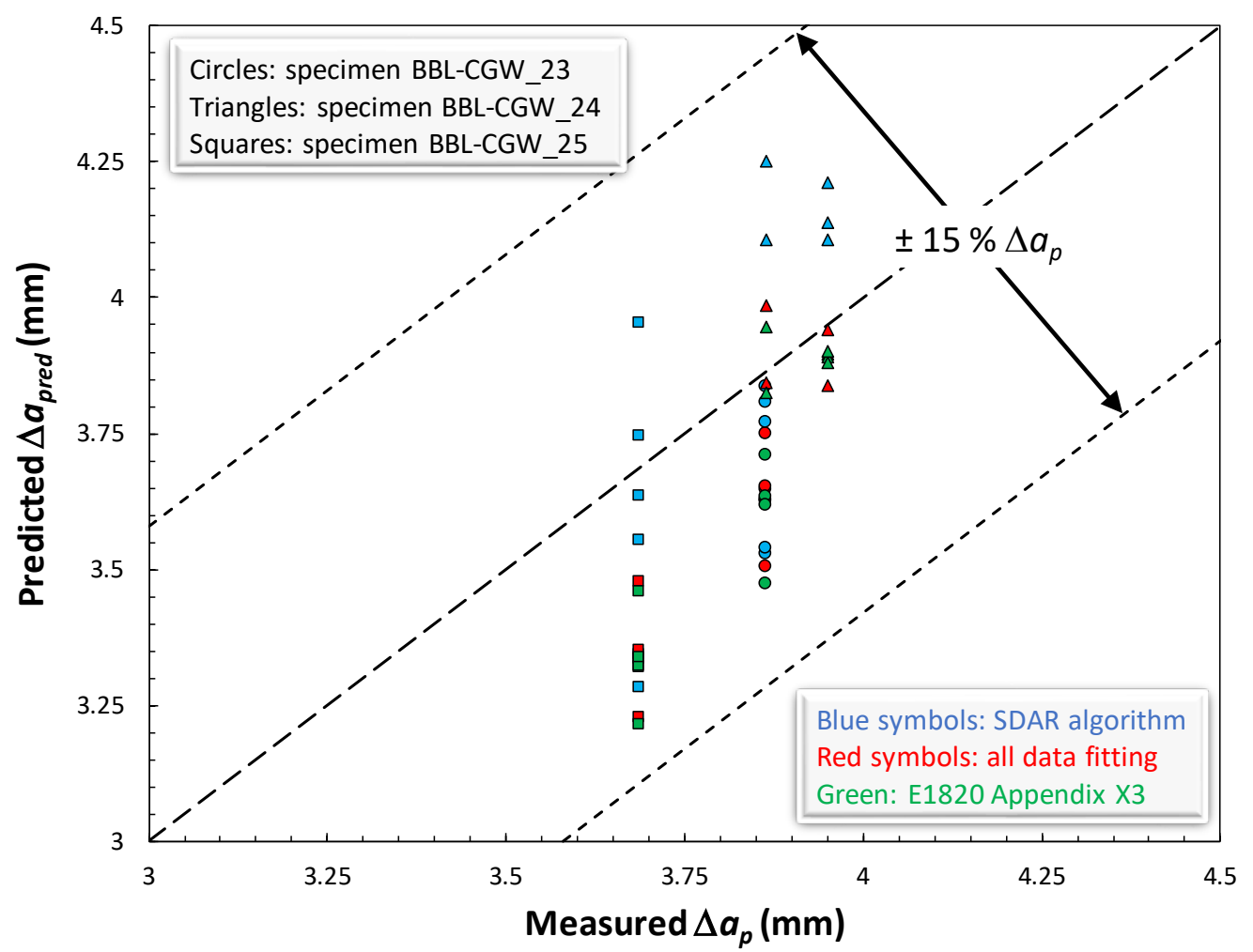

Fig. 16. Comparison between $\Delta a_{p}$ and $\Delta a_{\text {pred }}$ obtained according to ASTM E1820 App. X3, by fitting all data, and by means of SDAR. $\pm 15 \% \Delta a_{p}$ is the ASTM requirement. 


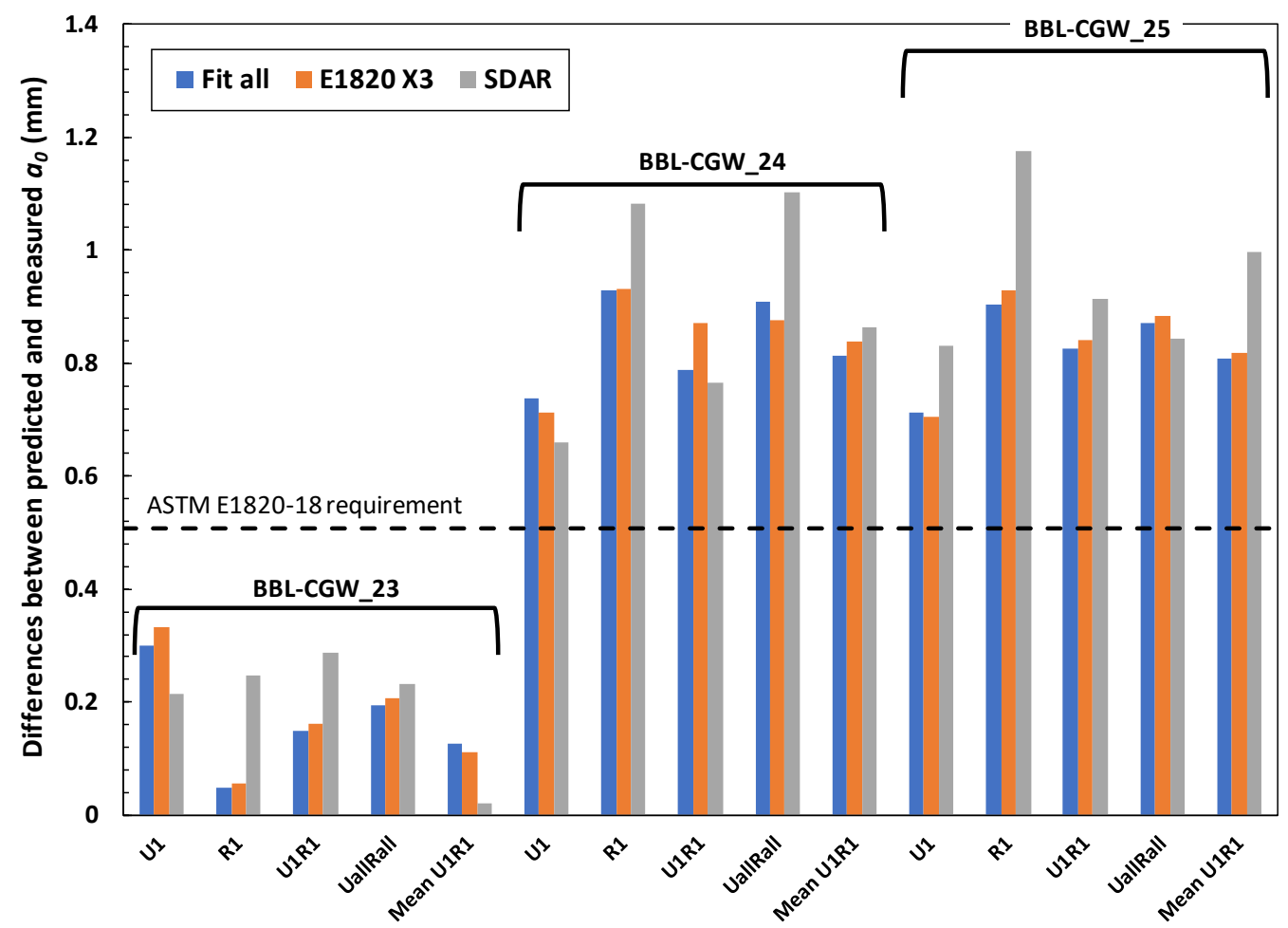

Fig. 17. Differences between predicted and measured initial crack sizes, and comparison with the ASTM E1820 requirement $( \pm 1 \% \mathrm{~W})$.

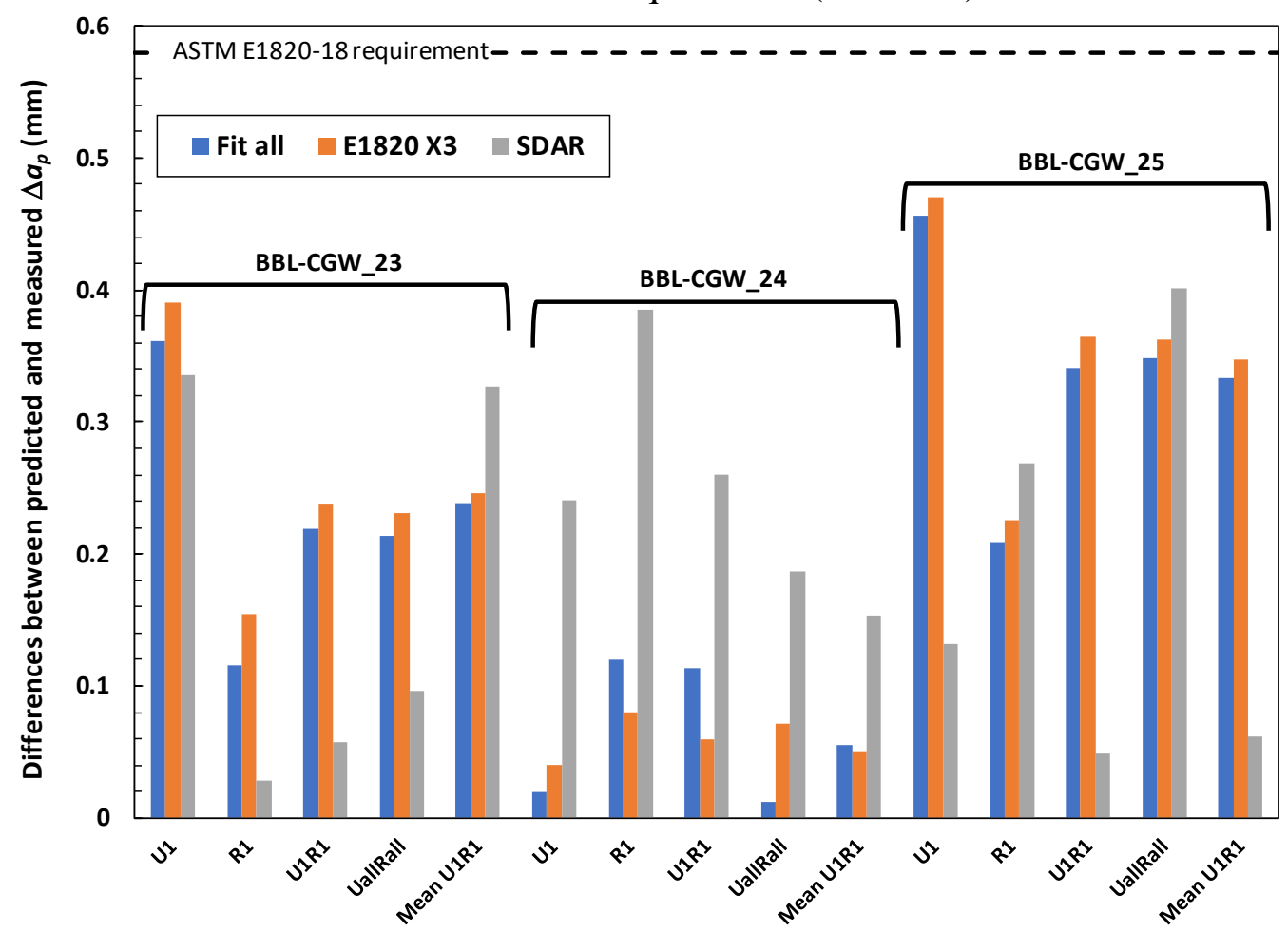

Fig. 18. Differences between predicted and measured total crack extensions, and comparison with the ASTM E1820 requirement $\left( \pm 15 \% \Delta a_{p}\right)$. 


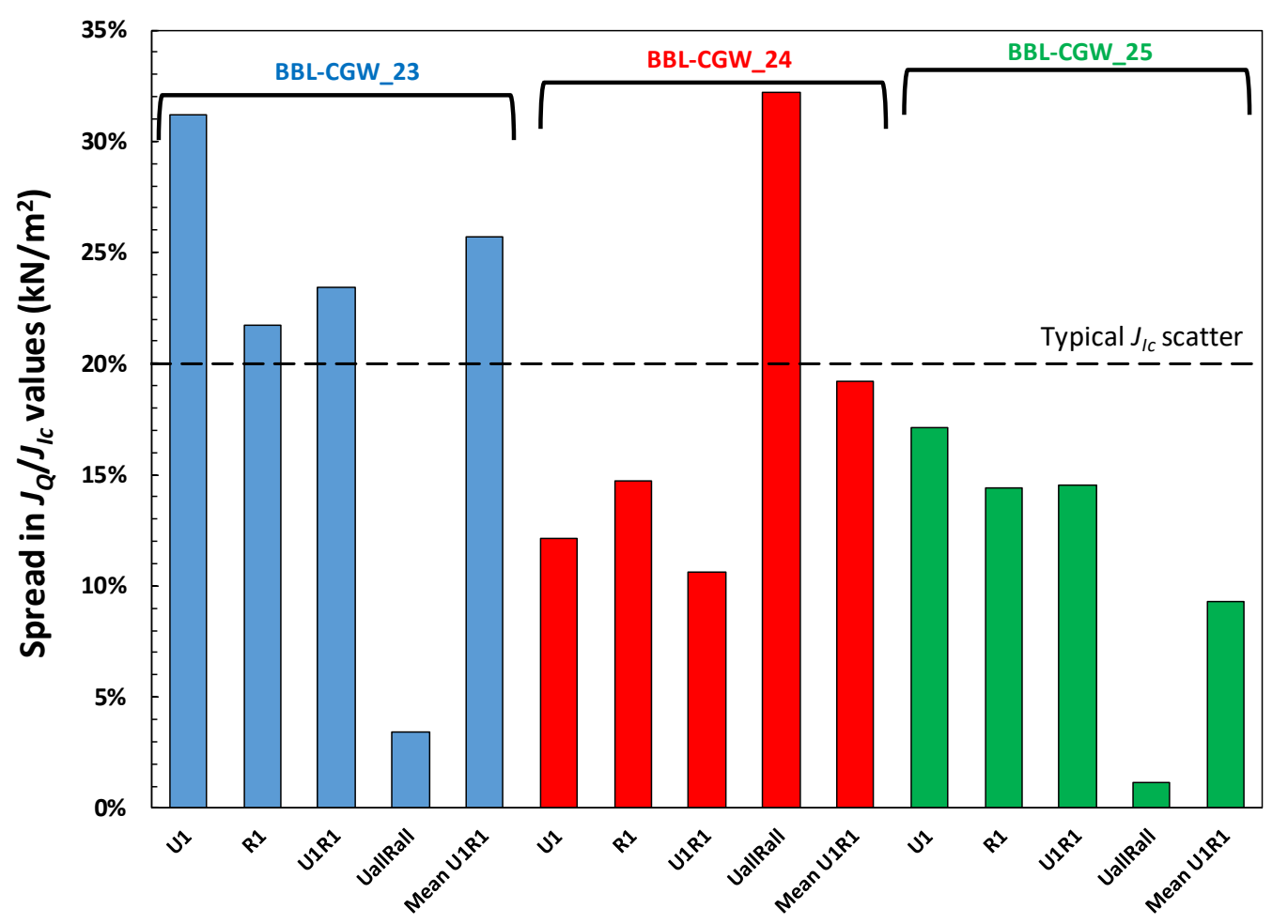

Fig. 19. Values of spread between minimum and maximum $J_{Q}$ obtained by the three approaches used (E1820 App. X3, fitting all data, SDAR algorithm).

(d) The comparison between $\left|a_{0}-a_{0 q}\right|$ values (Fig. 17) indicates that, in most cases, the largest differences are associated to the SDAR algorithm, irrespective of the data selected for the linear regression (U1, R1, U1R1, UallRall, Mean U1R1).

(e) However, the same can be said of the approach recommended ASTM E1820 App. X3 in regard to the total crack extension (Fig. 18), with the exception of specimen BBL-CGW_24, for which the SDAR algorithm yields by far the largest differences.

(f) The spread in $J_{Q}$ values, illustrated in Fig. 19, is mostly within the typical $\pm 20 \%$ scatter band, except for specimen BBL-CGW_23. No consistency for the different data selection criteria can be observed - for example, UallRall provides the lowest spread for two of the tests, and the highest for the third. The two extreme situations are illustrated in Fig. 20 (specimen BBL-CGW_24, UallRall: worst agreement) and Fig. 21 (specimen BBL-CGW_25, UallRall: worst agreement). Note that, in Fig. 20 and Fig. 21, the solid line is called construction line, the dotted lines represent E1820 crack extension validity limits, and the dashed line is the $0.2 \mathrm{~mm}$ offset construction line ( $J_{Q}$ is calculated at the intersection between this line and the power law fitting curve). 


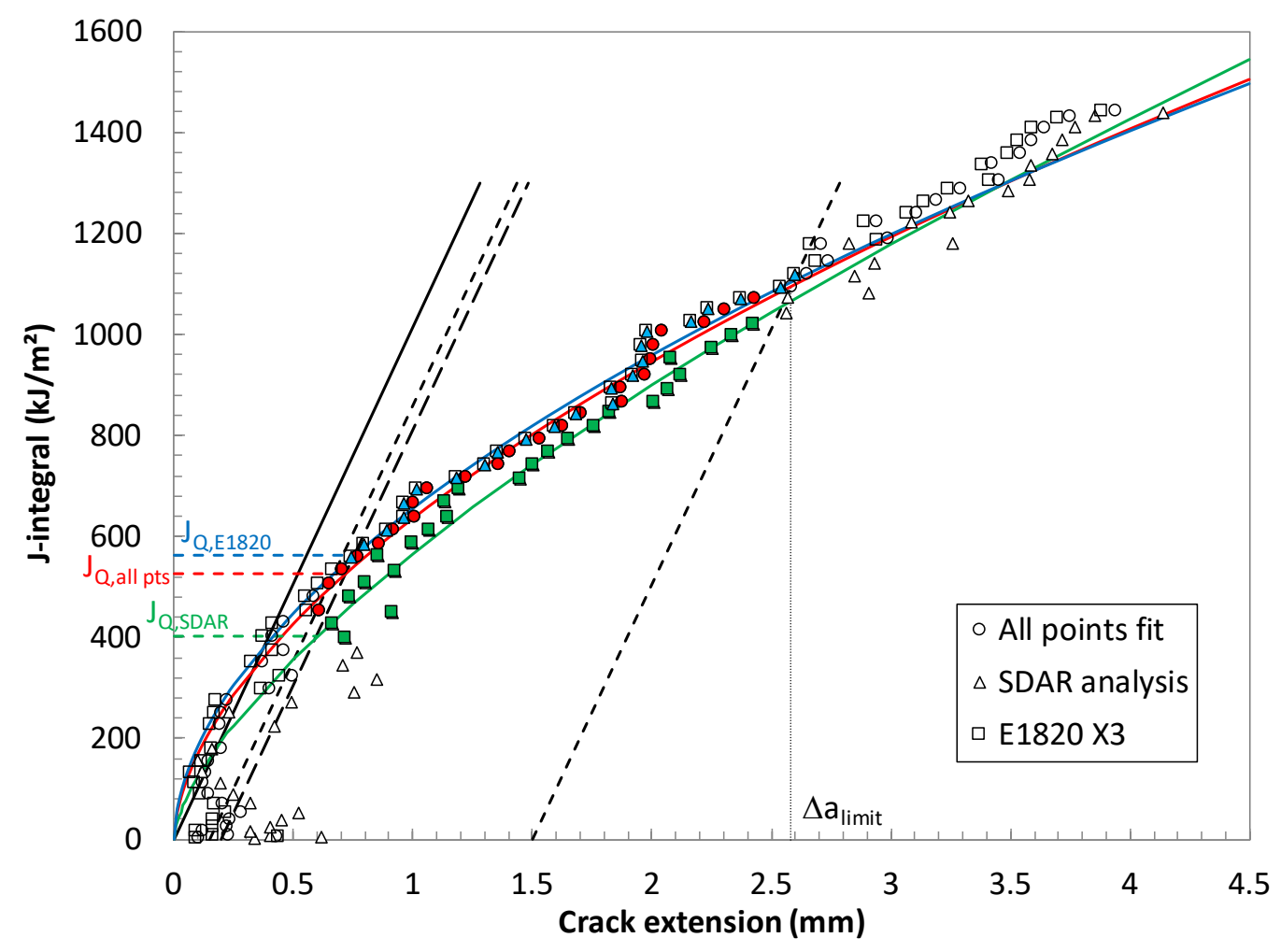

Fig. 20. Worst agreement: BBL-CGW_24, UallRall ( $J_{Q}$ spread: $\left.32.2 \%\right)$.

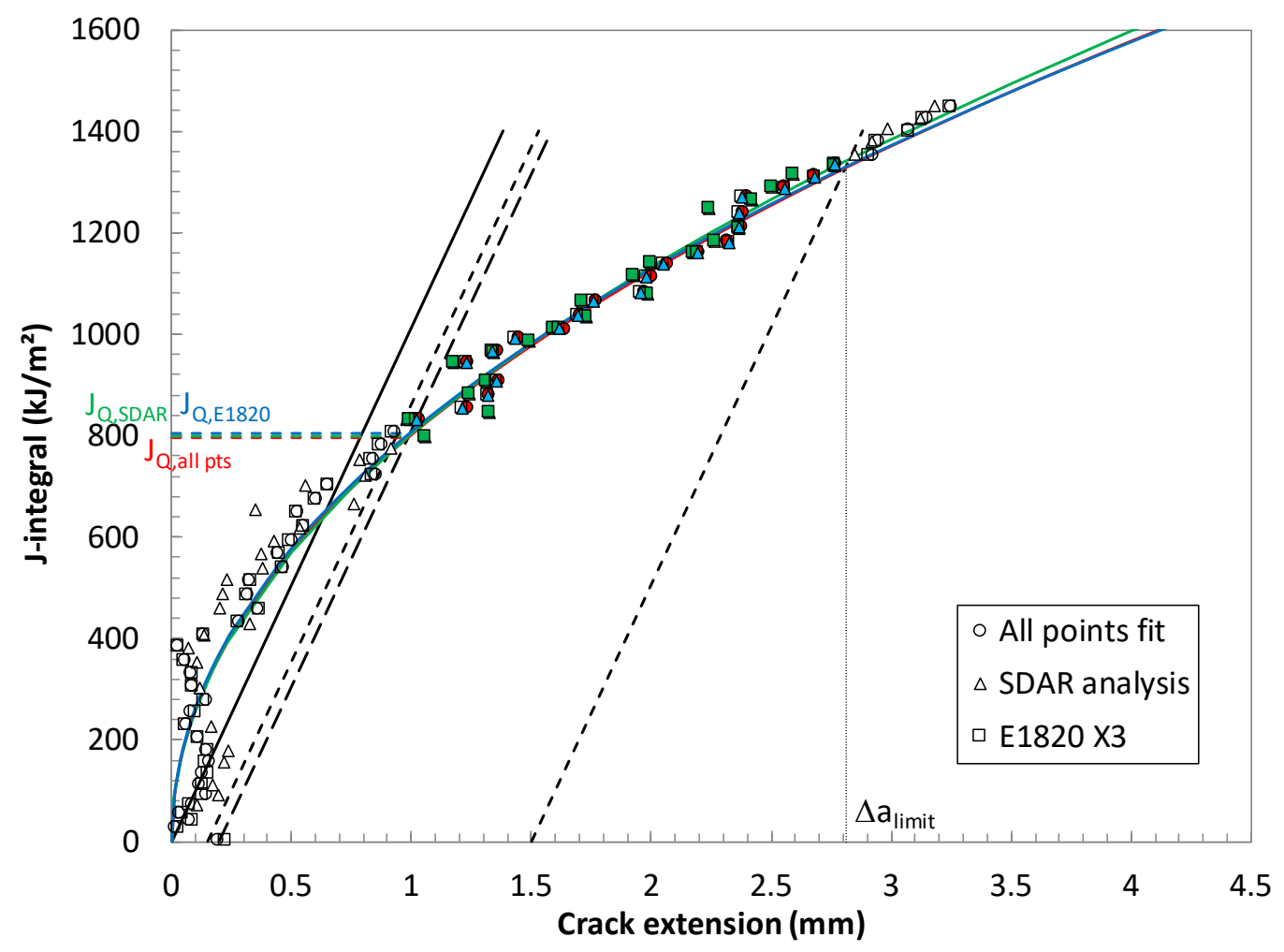

Fig. 21. Best agreement: BBL-CGW_25, UallRall ( $J_{Q}$ spread: $1.2 \%$ ). 


\subsubsection{ASTM round-robin on the analysis of standard data sets for fracture toughness evaluation (2013)}

In 2013, ASTM Task Group E08.07.05 on Ductile Fracture Toughness undertook an activity aimed at developing a set of standard data sets to help users validate their analysis programs. Within this analytical round-robin, eight data sets were distributed to participants, developed using $1 \mathrm{TC}(\mathrm{T})$ and $1 / 2 \mathrm{TC}(\mathrm{T})^{7}$ compact specimens, and 1TSE(B) single-edge bend specimens of an aluminum alloy and three steels [10].

In the original NIST round-robin submission, elastic compliance values had been calculated using all data points from both the unloading and reloading portions, without excluding points from the beginning or end of each cycle (i.e., not in accordance with the yet unpublished Appendix X3). In this study, we re-analyzed the eight data sets by applying the SDAR algorithm for the calculation of elastic compliances and the establishment of crack sizes.

The results, in terms of critical $J$-integral values $\left(J_{Q} / J_{I C}\right)$, estimated initial crack sizes $\left(a_{0 q}\right)$, and predicted crack extensions $\left(\Delta a_{\text {pred }}\right)$ are presented in Table 7 and Table 8, with SDAR values compared respectively to the original NIST submissions and to the mean round-robin values as reported in [10]. Relative differences are shown for $J$-integral values, while absolute differences are provided for crack sizes and crack extensions.

Table 7. Assessment of SDAR analyses with respect to the original NIST submission for the 2013 ASTM round-robin.

\begin{tabular}{|c|c|c|c|c|c|c|c|c|c|c|c|c|c|}
\hline \multirow{3}{*}{$\begin{array}{l}\text { File } \\
\text { ID }\end{array}$} & \multirow{3}{*}{$\begin{array}{c}\text { Specimen } \\
\text { type }\end{array}$} & & & & & & & & & & \multicolumn{3}{|c|}{$\begin{array}{l}\text { Differences with respect to } \\
\text { original NIST submission }\end{array}$} \\
\hline & & \multicolumn{3}{|c|}{$J_{Q} / J_{I C}\left(\mathrm{kN} / \mathrm{m}^{2}\right)$} & \multicolumn{3}{|c|}{$a_{o q}(\mathrm{~mm})$} & \multicolumn{3}{|c|}{$\Delta a_{\text {pred }}(\mathrm{mm})$} & $\Delta_{Q / l c}$ & $\Delta a_{o q}$ & $\Delta\left(\Delta a_{\text {pred }}\right)$ \\
\hline & & Original & SDAR & Mean $\mathrm{R}-\mathrm{R}$ & Original & SDAR & Mean R-R & Original & SDAR & Mean $\mathrm{R}-\mathrm{R}$ & (\%) & $(\mathrm{mm})$ & $(\mathrm{mm})$ \\
\hline EUR-U26 & $1 \mathrm{TC}(\mathrm{T})$ & 393.4 & 389.4 & 385.6 & 29.63 & 29.65 & 29.65 & 2.58 & 2.60 & 2.60 & $-1.0 \%$ & 0.01 & 0.03 \\
\hline EUR-U44 & $1 \mathrm{TC}(\mathrm{T})$ & 447.6 & 431.7 & 418.4 & 28.76 & 28.76 & 28.75 & 2.67 & 2.70 & 2.70 & $-3.6 \%$ & 0.00 & 0.03 \\
\hline FYB-A1 & $1 \mathrm{TC}(\mathrm{T})$ & 171.0 & 102.2 & 146.3 & 30.57 & 30.41 & 30.63 & 13.57 & 13.81 & 13.53 & $-40.3 \%$ & -0.17 & 0.25 \\
\hline FYB-94G & 1TSE(B) & 194.0 & 200.4 & 193.1 & 27.55 & 27.60 & 27.55 & 7.11 & 7.19 & 7.11 & $3.3 \%$ & 0.05 & 0.07 \\
\hline GJO-12A & $1 \mathrm{TC}(\mathrm{T})$ & 347.5 & 266.2 & 297.8 & 28.84 & 28.72 & 28.46 & 3.29 & 3.39 & 3.43 & $-23.4 \%$ & -0.12 & 0.10 \\
\hline FGN-10 & 1TSE(B) & 38.5 & 36.7 & 38.6 & 31.21 & 31.21 & 31.21 & 10.73 & 10.76 & 10.76 & $-4.5 \%$ & 0.00 & 0.04 \\
\hline FGN-30 & $1 \mathrm{TC}(\mathrm{T})$ & 32.6 & 32.6 & 33.7 & 30.23 & 30.23 & 30.26 & 12.50 & 12.50 & 12.48 & $0.0 \%$ & 0.00 & 0.00 \\
\hline FGN-74 & $1 / 2 \mathrm{TC}(\mathrm{T})$ & 34.4 & 33.8 & 34.2 & 15.07 & 15.10 & 15.07 & 7.47 & 7.48 & 7.48 & $-1.6 \%$ & 0.03 & 0.01 \\
\hline
\end{tabular}

\footnotetext{
${ }^{7}$ Thickness $=1 / 2$ in. $=12.7 \mathrm{~mm}$.
} 
Table 8. Assessment of SDAR analyses with respect to mean participants' results from the 2013 ASTM round-robin.

\begin{tabular}{|c|c|c|c|c|c|c|c|c|c|c|c|c|c|}
\hline \multirow{3}{*}{$\begin{array}{l}\text { File } \\
\text { ID }\end{array}$} & \multirow{3}{*}{$\begin{array}{c}\text { Specimen } \\
\text { type }\end{array}$} & \multirow{2}{*}{\multicolumn{3}{|c|}{$J_{Q} / J_{I C}\left(\mathrm{kN} / \mathrm{m}^{2}\right)$}} & \multirow{2}{*}{\multicolumn{3}{|c|}{$a_{O q}(\mathrm{~mm})$}} & \multirow{2}{*}{\multicolumn{3}{|c|}{$\Delta a_{\text {pred }}(\mathrm{mm})$}} & \multicolumn{3}{|c|}{$\begin{array}{c}\text { Differences with respect to } \\
\text { mean R-R results }\end{array}$} \\
\hline & & & & & & & & & & & $\Delta_{Q / l c}$ & $\Delta a_{O q}$ & $\Delta\left(\Delta a_{\text {pred }}\right)$ \\
\hline & & Original & SDAR & Mean R-R & Original & SDAR & Mean R-R & Original & SDAR & Mean R-R & (\%) & $(\mathrm{mm})$ & $(\mathrm{mm})$ \\
\hline EUR-U26 & $1 \mathrm{TC}(\mathrm{T})$ & 393.4 & 389.4 & 385.6 & 29.63 & 29.65 & 29.65 & 2.58 & 2.60 & 2.60 & $1.0 \%$ & 0.00 & 0.00 \\
\hline EUR-U44 & $1 \mathrm{TC}(\mathrm{T})$ & 447.6 & 431.7 & 418.4 & 28.76 & 28.76 & 28.75 & 2.67 & 2.70 & 2.70 & $3.2 \%$ & -0.01 & 0.00 \\
\hline FYB-A1 & $1 \mathrm{TC}(\mathrm{T})$ & 171.0 & 102.2 & 146.3 & 30.57 & 30.41 & 30.63 & 13.57 & 13.81 & 13.53 & $-30.2 \%$ & 0.22 & 0.28 \\
\hline FYB-94G & 1TSE(B) & 194.0 & 200.4 & 193.1 & 27.55 & 27.60 & 27.55 & 7.11 & 7.19 & 7.11 & $3.8 \%$ & -0.05 & 0.08 \\
\hline GJO-12A & $1 \mathrm{TC}(\mathrm{T})$ & 347.5 & 266.2 & 297.8 & 28.84 & 28.72 & 28.46 & 3.29 & 3.39 & 3.43 & $-10.6 \%$ & -0.26 & -0.04 \\
\hline $\mathrm{FGN}-10$ & 1TSE(B) & 38.5 & 36.7 & 38.6 & 31.21 & 31.21 & 31.21 & 10.73 & 10.76 & 10.76 & $-4.9 \%$ & 0.00 & 0.00 \\
\hline FGN-30 & $1 \mathrm{TC}(\mathrm{T})$ & 32.6 & 32.6 & 33.7 & 30.23 & 30.23 & 30.26 & 12.50 & 12.50 & 12.48 & $-3.4 \%$ & 0.03 & 0.02 \\
\hline FGN-74 & $1 / 2 \mathrm{TC}(\mathrm{T})$ & 34.4 & 33.8 & 34.2 & 15.07 & 15.10 & 15.07 & 7.47 & 7.48 & 7.48 & $-1.0 \%$ & -0.03 & 0.00 \\
\hline
\end{tabular}

\subsubsection{Discussion}

Examination of both Table 7 and Table 8 shows that, for six out of eight data sets, the use of the SDAR algorithm produces negligible differences with respect to the original NIST submission and the mean round-robin results. For these tests, $J_{Q} / J_{I c}$ variations are lower than $5 \%$, and crack size/extension differences are within $0.05 \mathrm{~mm}$.

For the remaining two data sets (specimen FYB-A1, 1TC(T) specimen of HY-80 steel, and specimen GJO-12A, 1TC(T) specimen of A533B steel), however, discrepancies are more significant. $J-R$ curves from the original NIST submission and obtained from the SDAR analyses are shown in Fig. 22 (FYB-A1) and Fig. 23 (GJO-12A). For comparison purposes, a similar $J$ - $R$ curve comparison is provided in Fig. 24 for the data set with the lowest discrepancies, EUR-U26 (1TC(T) specimen of A508 Cl. 2). 


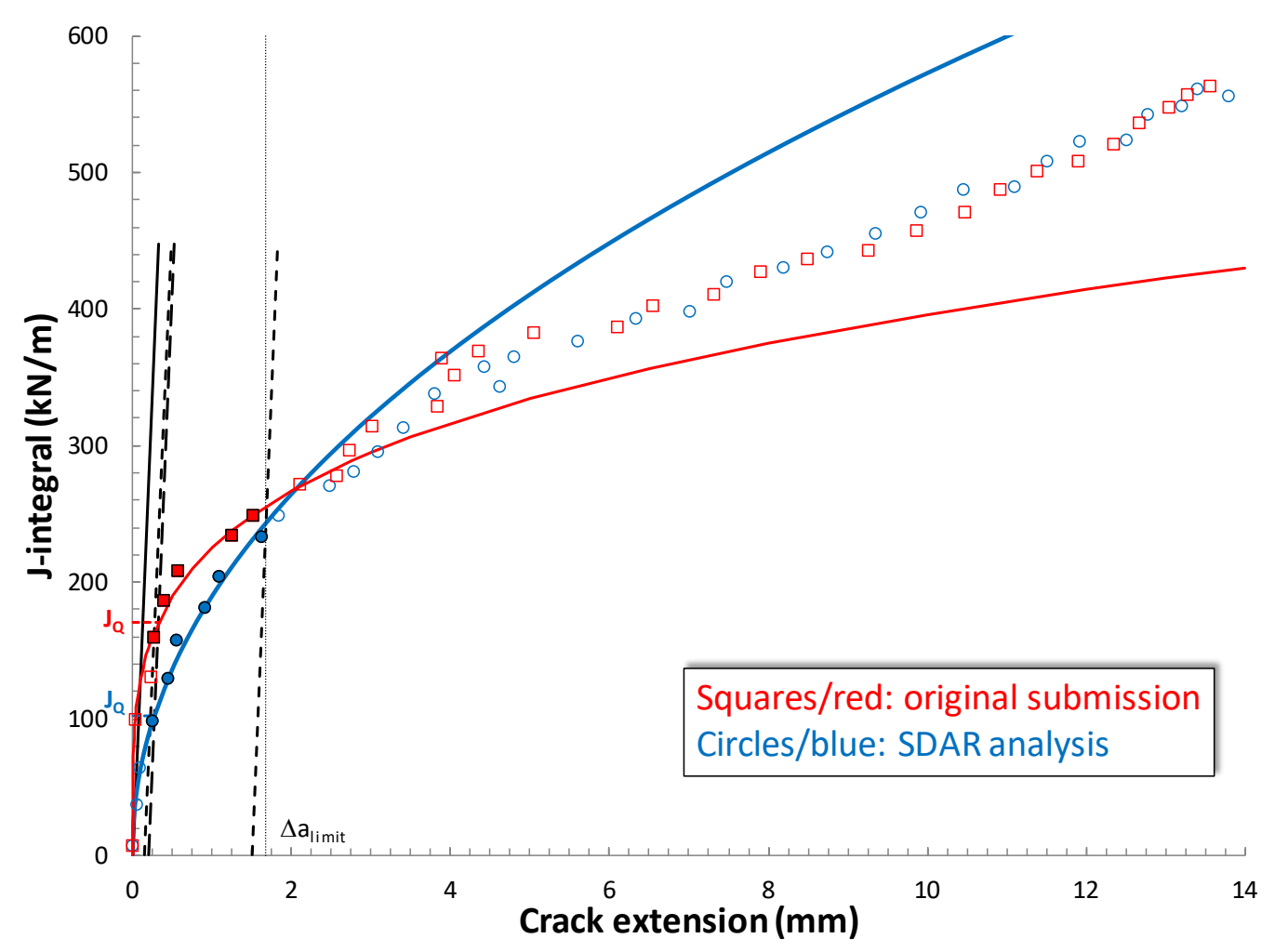

Fig. 22. Worst agreement: FYB-A1.

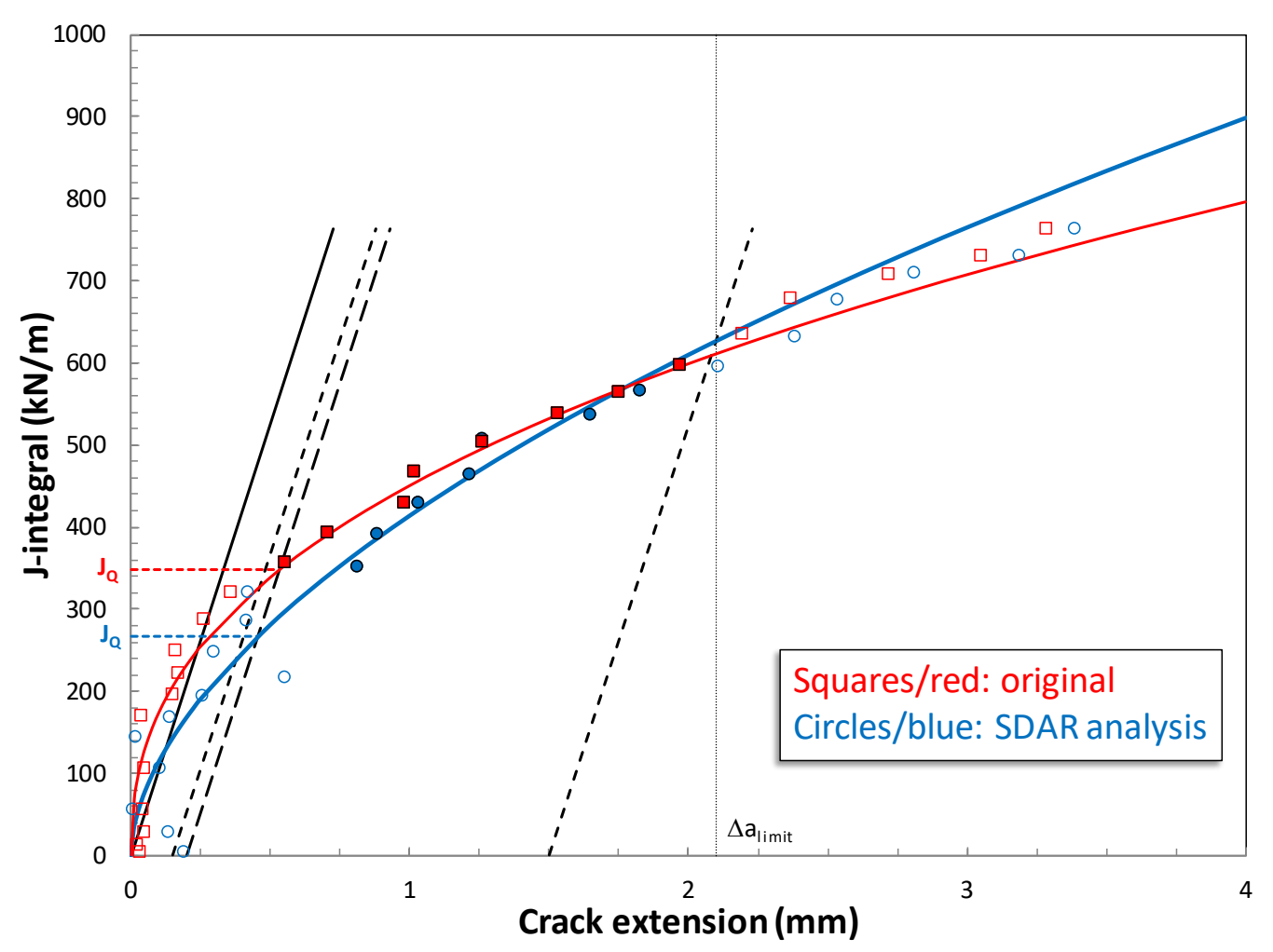

Fig. 23. Unsatisfactory agreement: GJO-12A. 


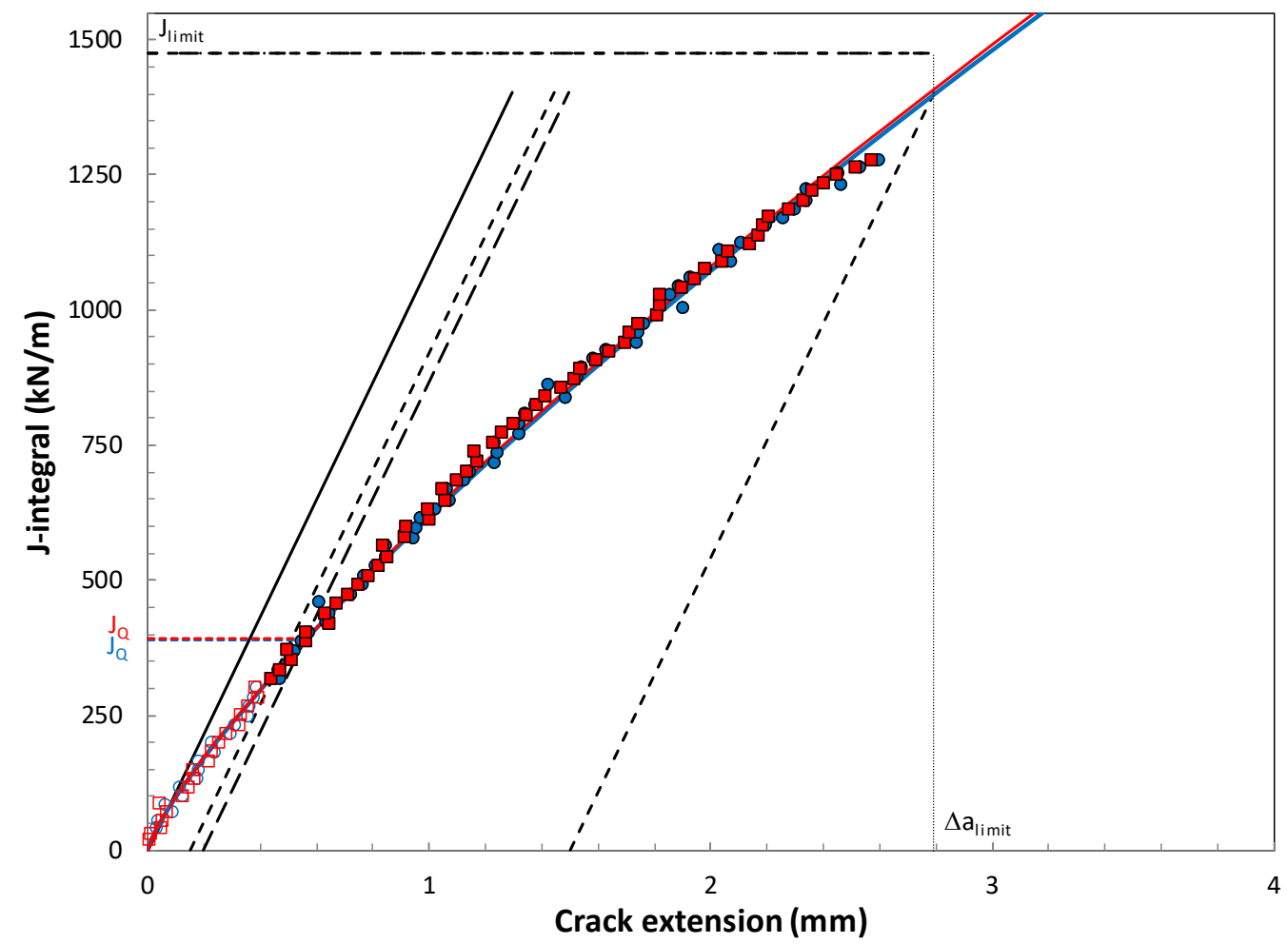

Fig. 24. Best agreement: EUR-U26.

Some insight into the correlation between original and SDAR analyses can be gained by examining the level of experimental noise and hysteresis for the unloading cycles of these data sets. To this purpose, we illustrated the unloading cycles corresponding to the maximum force for FYB-A1 (Fig. 25 - pronounced noise and hysteresis), GJO-12A (Fig. 26 - significant noise and hysteresis), and EUR-U26 (Fig. 27 - low noise and hysteresis).

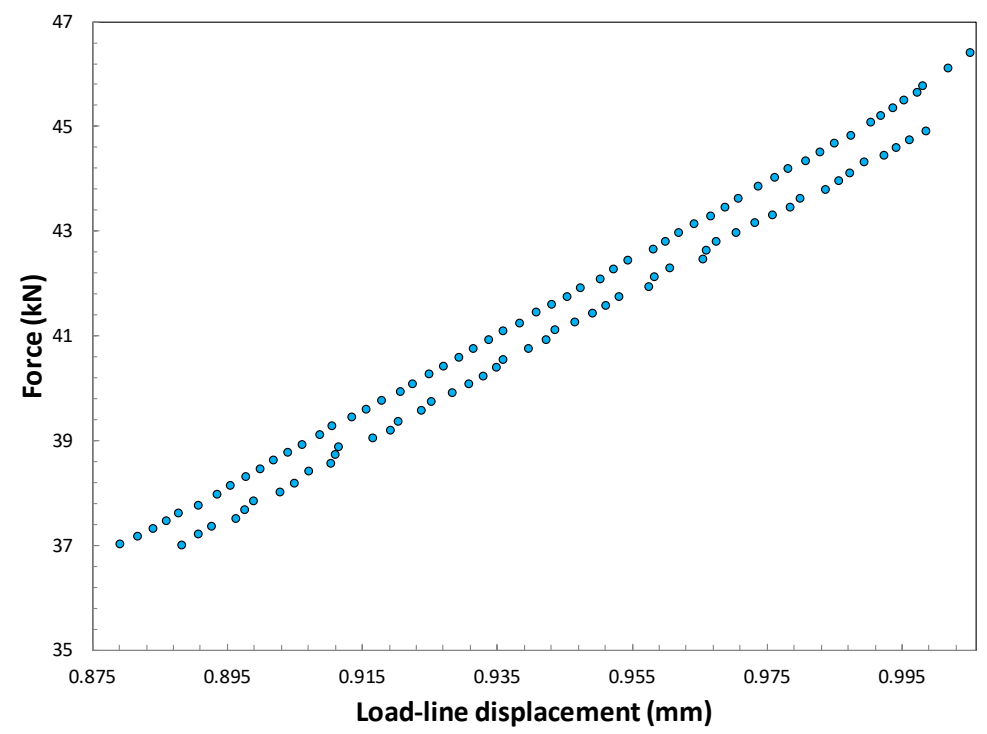

Fig. 25. Pronounced noise and hysteresis: maximum force unloading cycle for FYB-A1. 


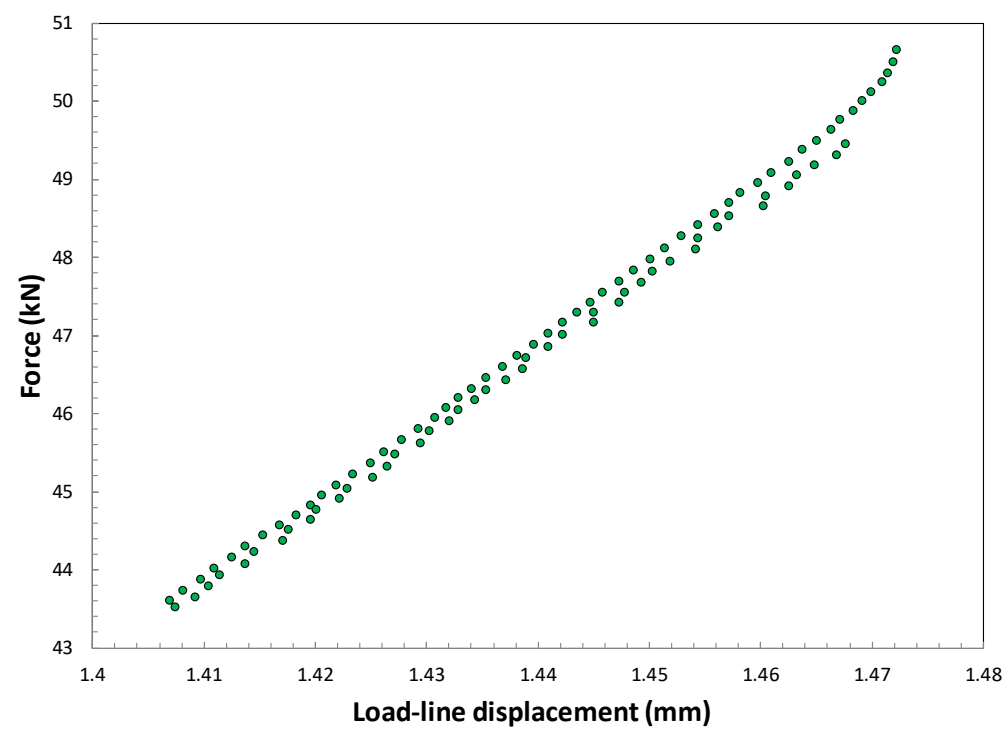

Fig. 26. Significant noise and hysteresis: maximum force unloading cycle for GJO-12A.

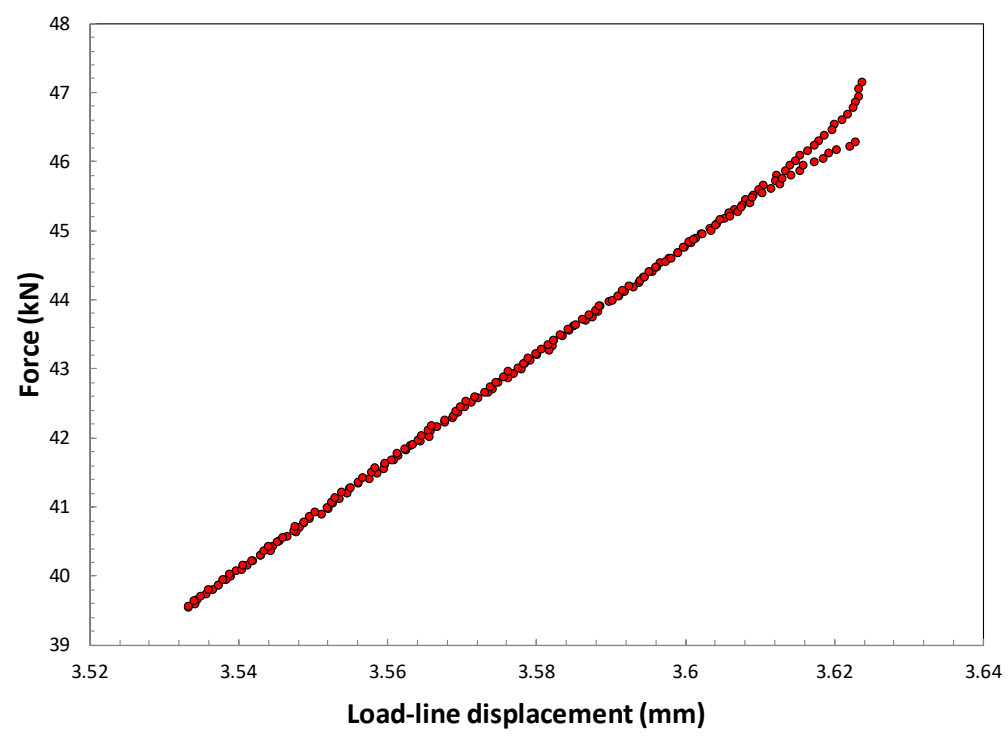

Fig. 27. Low noise and hysteresis: maximum force unloading cycle for EUR-U26.

It appears that the higher the level of noise and hysteresis of the unloading cycle, the more likely the SDAR algorithm is to yield a linear slope that is not truly representative of the actual elastic behavior of the sample. This issue can be further aggravated by the relatively small window size required by E3076 for SDAR to run (larger between 10 points or $20 \%$ of the original data set). If this minimum size were to be increased (for example to $50 \%$ ), it is possible that the agreement between SDAR and Appendix X3 of E1820 would improve, even in case of noisy data sets. An example is shown in Fig. 28 for specimen BBL-CGW_23 of the HZDR round-robin: the $J_{Q}$ value obtained by setting the minimum window size at $50 \%$ is much closer to the result obtained from E1820 Appendix X3 than if the minimum window size is kept at $20 \%$ as in E3076. 
Furthermore, algorithms for “cleaning up” sets are relatively common, and they could be implemented as a possible preliminary step in a future revision of the ASTM E3076 standard.

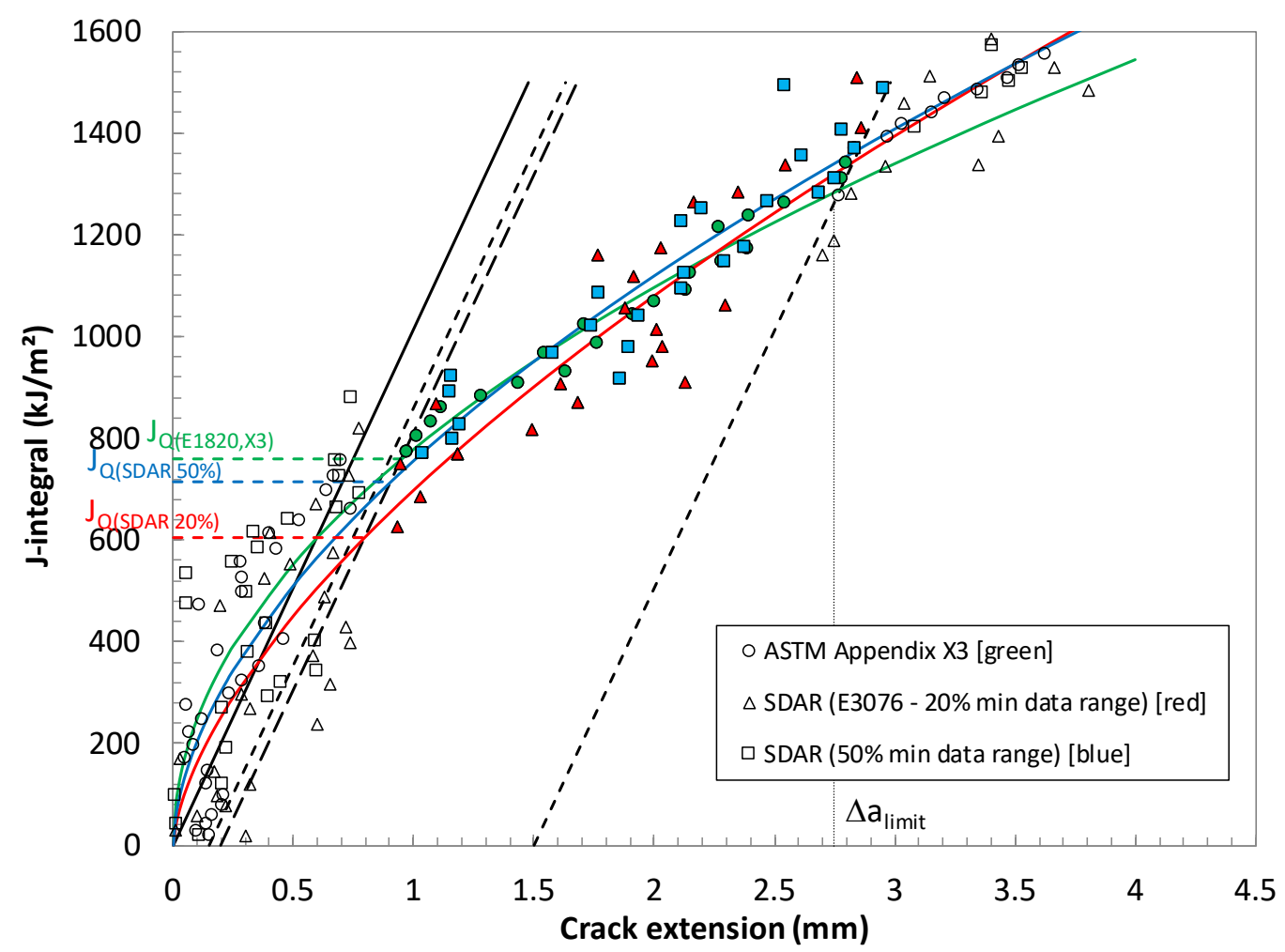

Fig. 28 - Specimen BBL-CGW_23 (HZDR round-robin): comparison between ASTM E1820 App. X3 analysis and SDAR analyses (with $20 \%$ and $50 \%$ minimum window size).

A closer look at the unloadings for specimen FYB-A1 (Fig. 29) reveals that for 10 of its 36 unloading cycles, SDAR used fewer than $30 \%$ of the data points to calculate the compliance. On the average, $38 \%$ of the unloading cycle data points were used for compliance calculation. 


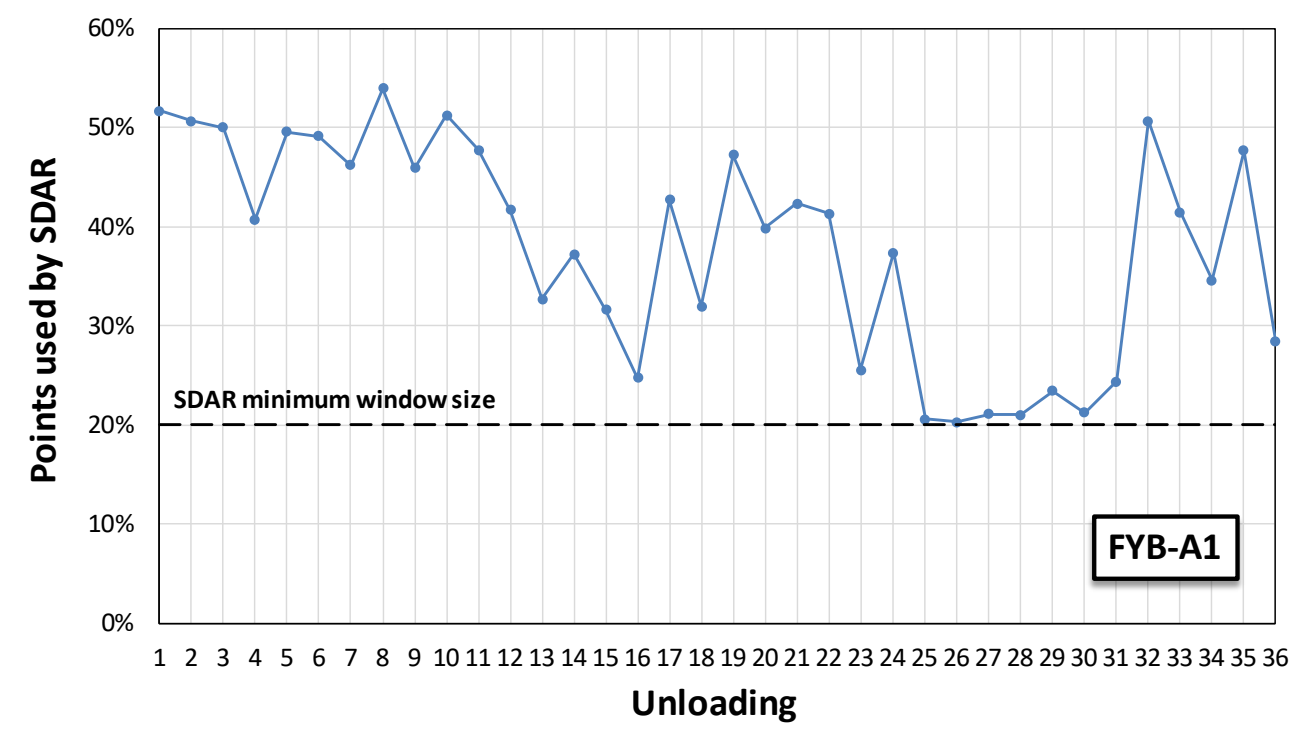

Fig. 29. Specimen FYB-A1: percentage of unloading cycle data points used by SDAR.

Conversely, for the "best" specimen EUR-U26, a larger fraction of data points from each unloading were used for compliance calculation (Fig. 30): on the average, $46 \%$, significantly more than the threshold prescribed by ASTM E3076-18 (20 \%). This guarantees a better representation of the overall elastic behavior of the sample during each unloading cycle.

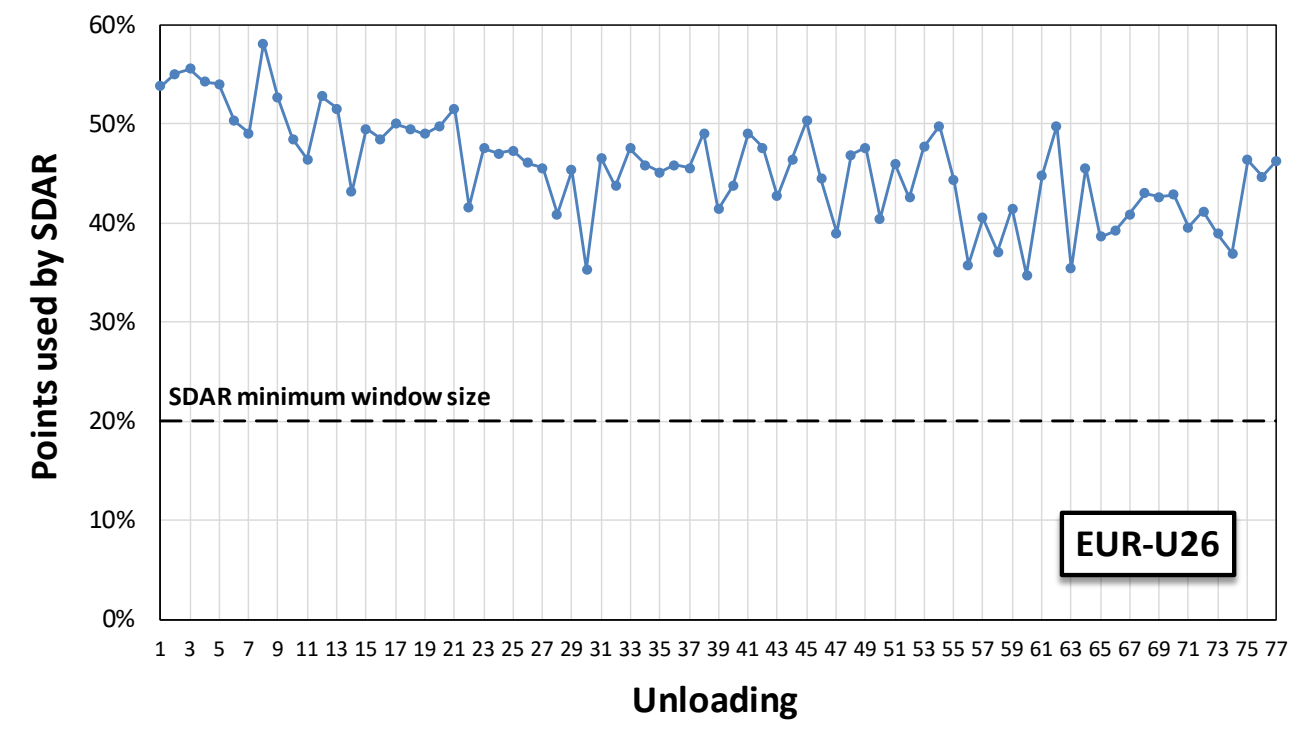

Fig. 30. Specimen EUR-U26: percentage of unloading cycle data points used by SDAR.

The use of the SDAR algorithm for the calculation of compliance had little effect on the determination of the critical toughness, as shown in Fig. 31. Except for specimen FYB94G, the value of $J_{Q} / J_{I c}$ from SDAR is lower than that obtained in the original analysis (fitting all data points). This is consistent with the findings reported in 4.1.1.1 (item b). 
Also, with the exception of FGN-10 and FYB-A1, both the results from SDAR and the original analysis are within the standard deviations of the round-robin submissions (indicated as error bars in the Figure).
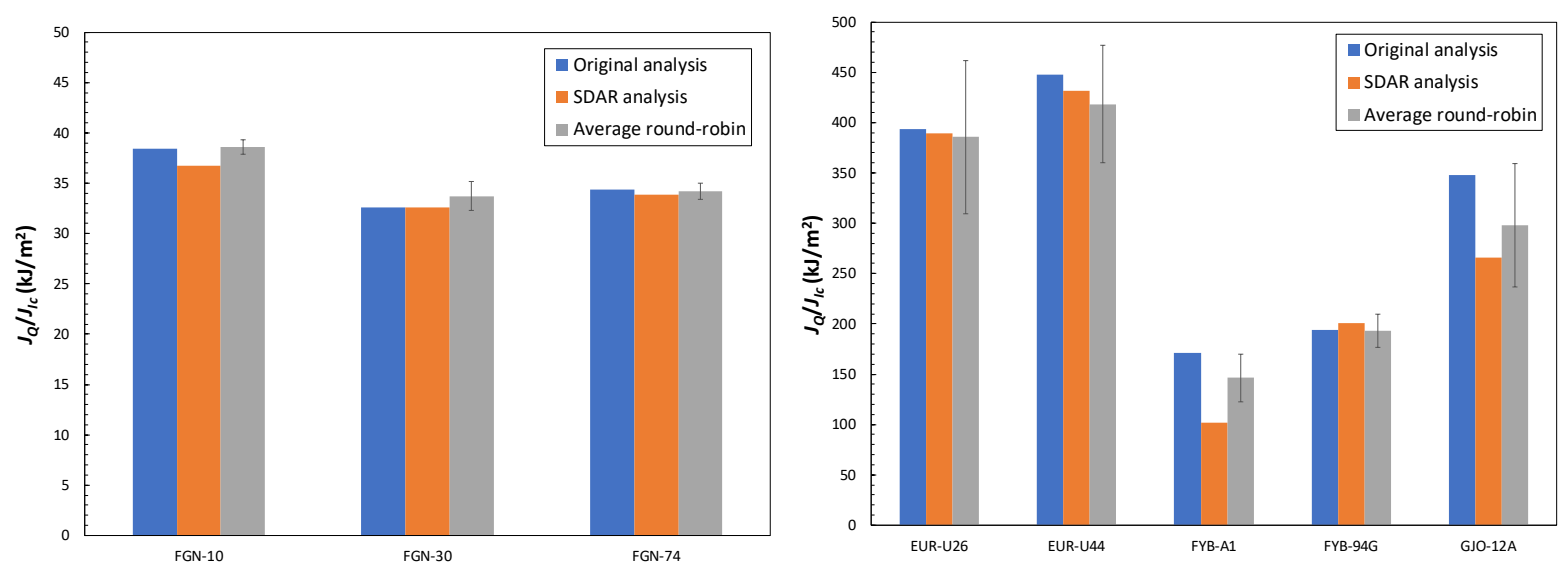

Fig. 31. Comparison between critical toughness values for the ASTM round-robin data sets.

In terms of crack extension (Fig. 32), SDAR consistently yields larger $\Delta a_{\text {pred }}$ values than the original analyses. In most cases, both types of prediction fall within the scatter band of the round-robin submission. Again, this is consistent with what we reported in 4.1.1.1 (item c). It's interesting to note that all predictions are smaller than the measured $\Delta a_{p}$ values, except for specimen FGN-74.
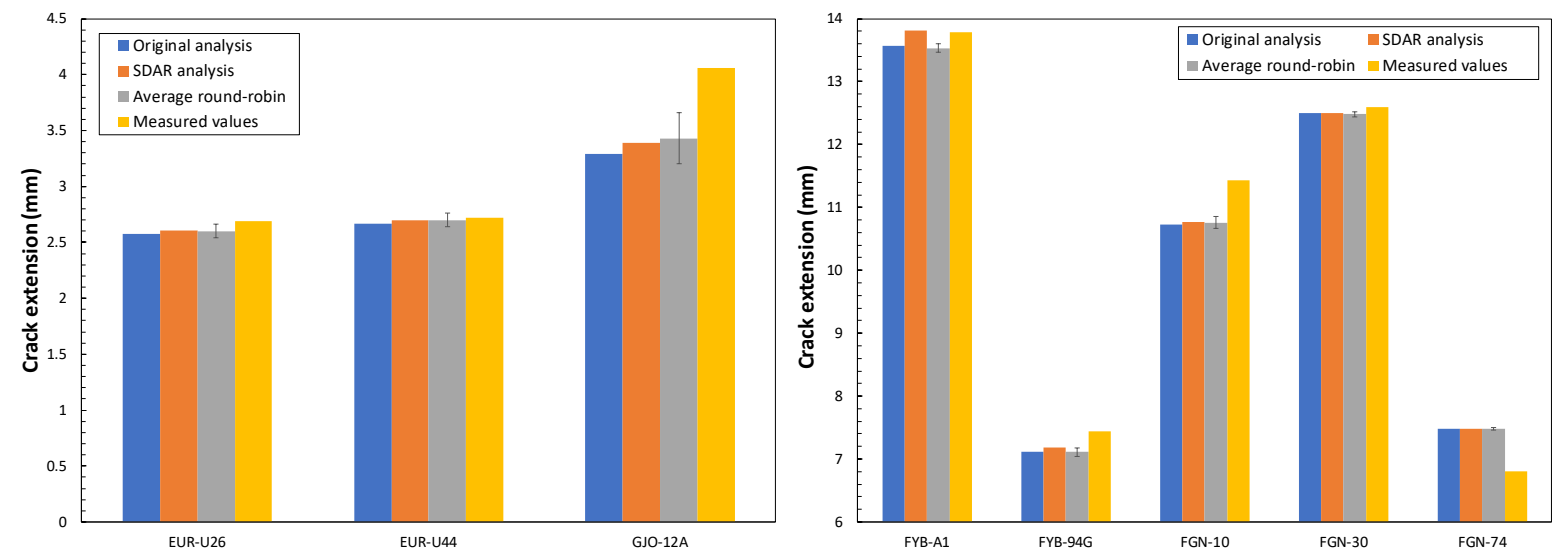

Fig. 32. Comparison between measured and predicted values of final crack extension for the ASTM round-robin data sets.

\section{Conclusions}

Although in most cases the results obtained by using the SDAR algorithm for the determination of elastic compliance will be substantially equivalent to those obtained by applying the recommendations of Appendix X3 of the current ASTM E1820-18a standard, there might be issues in case of very noisy data sets and/or large hysteresis between unloading and reloading sections. Under these circumstances, the most linear part of the data set identified by SDAR might not accurately represent the true elastic behavior of the fracture toughness (or fatigue) specimen, particularly if the minimum number of data points is kept at 
the same level presently standardized in ASTM E3076-18 (20\% of the points in the search range or 10 points, whichever is greater). Better accuracy could be achieved by increasing this threshold to at least $50 \%$.

Therefore, the use of SDAR as described in E3076 is not recommended for elastic compliance and crack size calculations in fracture toughness or fatigue crack growth tests.

Despite this, the SDAR algorithm remains an extremely valuable tool for establishing the slope of the most linear region of a test in many mechanical applications (tensile tests, linear elastic fracture mechanics tests, etc.), and its use is strongly recommended.

\section{References}

[1] ASTM E8/E8M-16a, Standard Test Methods for Tension Testing of Metallic Materials, ASTM Book of Standards, Vol. 03.01 (Mechanical Testing of Metals), American Society for Testing and Materials, West Conshohocken, PA.

[2] ASTM E399-17, Standard Test Methods for Linear-Elastic Plane-Strain Fracture Toughness $K_{\text {Ic }}$ of Metallic Materials, ASTM Book of Standards, Vol. 03.01 (Mechanical Testing of Metals), American Society for Testing and Materials, West Conshohocken, PA.

[3] ASTM E1820-18, Standard Test Method for Measurement of Fracture Toughness, ASTM Book of Standards, Vol. 03.01 (Mechanical Testing of Metals), American Society for Testing and Materials, West Conshohocken, PA.

[4] ASTM E647-15 ${ }^{1}$, Standard Test Method for Measurement of Fatigue Crack Growth Rates, ASTM Book of Standards, Vol. 03.01 (Mechanical Testing of Metals), American Society for Testing and Materials, West Conshohocken, PA.

[5] McKeighan PC, Hillberry BM (1991) An Algorithm for Determining Tensile Properties from Digitally Recorded Tensile Test Data. Exp. Tech., Vol. 15, No. 6, pp. 27-30.

[6] Roebuck B, Lord JD, Cooper PM, McCartney, LN (1994) Data Acquisition and Analysis of Tensile Properties for Metal Matrix Composites. J. Test. Eval., Vol. 22, No. 1, pp. 63-69.

[7] Scibetta M, Schuurmans, J (2004) Development and Qualification of an Algorithm for the Determination of the Initial Linear Portion of a Force Versus Displacement Record. $J$. Test. Eval., Vol 32, No. 6, pp. 500-503.

[8] Graham SM, Adler MA (2011) Determining the Quality of Fit for the Linear Part of a Test Record. J. Test. and Eval., Vol. 39, No. 2, pp. 260-268.

[9] Graham SM (smgraham@usna.edu), email communication to E. Lucon, 3/29/2018.

[10] Link, RE (2015) Round-robin Analysis of Standard Data Sets for Fracture Toughness Evaluation in ASTM E1820. J. Test. and Eval., Vol. 43, No. 1, pp. 159-170.

[11] Graham, SM (2014) Uncertainty in Ductile Fracture Initiation Toughness $\left(J_{I c}\right)$ Resulting From Compliance Measurement. Application of Automation Technology in Fatigue and Fracture Testing and Analysis, STP 1571, PC McKeighan and AA Braun, Eds., pp. 134 - 152, ASTM International, West Conshohocken, PA. 


\section{Appendix A: Visual Basic code of the Excel subroutine "Regression"}

Attribute VB_Name = "Module2"

Sub Regression( )

Dim Numpoints As Integer

Dim Window_size As Integer

Dim nfit As Long

Dim xnorm(2000) As Variant

Dim ynorm(2000) As Variant

Dim xfit(2000) As Variant

Dim yfit(2000) As Variant

Dim StartTime As Double

Dim EndTime As Double

nrmin $=9999$

mfit $=0$

bfit $=\odot$

StartTime $=$ Timer

Application.Calculation $=$ xlCalculationManual

Range("L3:L7").ClearContents

' Read input data

Numpoints = Range ("K10").Value

Window_size = Range ("K11"). Value

For $i=1$ To Numpoints xnorm( $i)=$ Range ("D" \& $i+2)$. Value

Next $i$ $\operatorname{ynorm}(i)=$ Range $(" E " \& i+2)$. Value

' Regression

startfit $=1$

endfit $=$ Window_size

Application.ScreenUpdating = False

$1 \operatorname{sum} x=0$

sumy $=0$

diffxy $=0$

diffsq $=0$

$j=1$

For $i=$ startfit To endfit

$\operatorname{xfit}(j)=\operatorname{xnorm}(i)$

$\operatorname{sum} x=\operatorname{sum} x+x f i t(j)$

$\operatorname{yfit}(j)=\operatorname{ynorm}(i)$ 


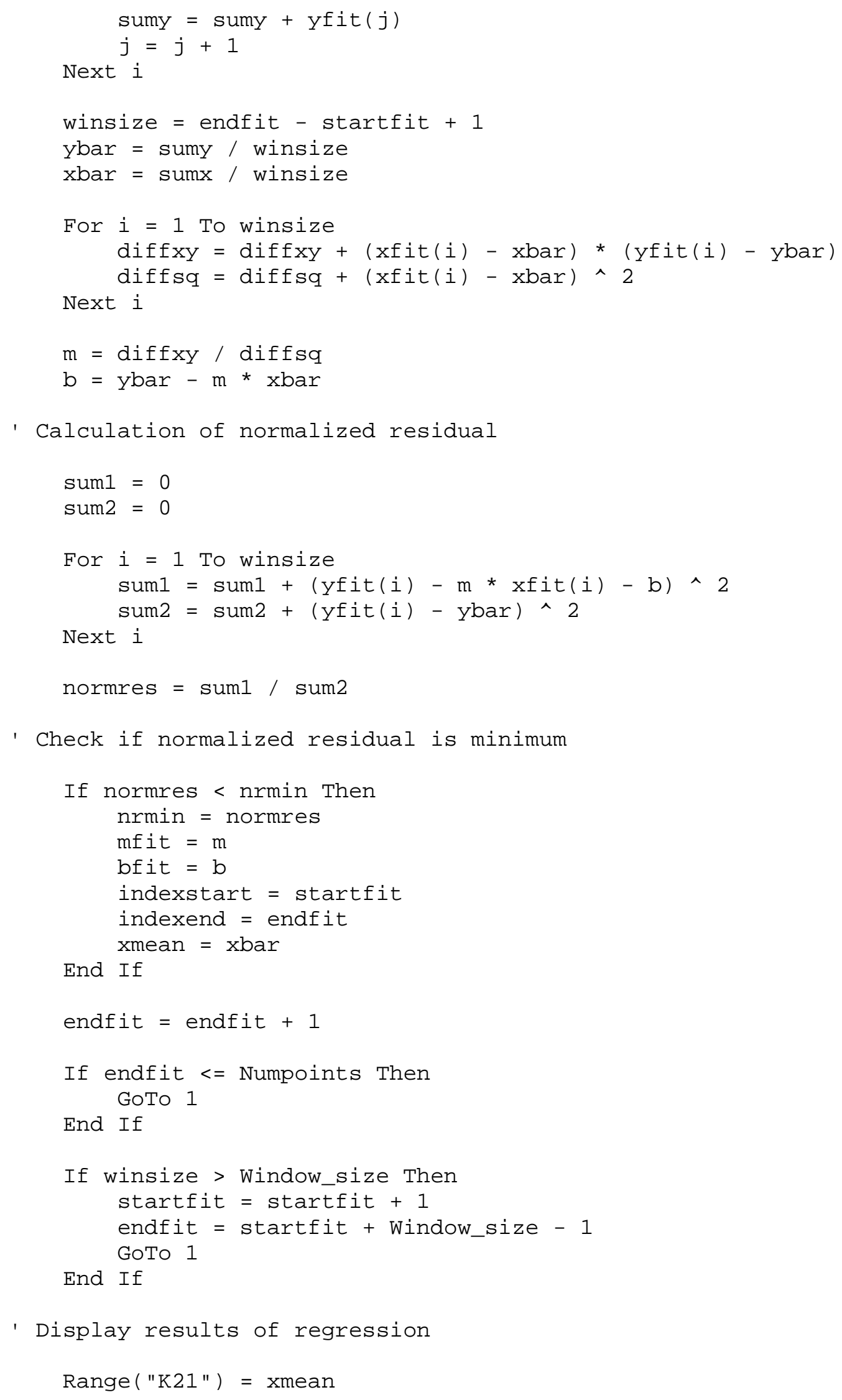




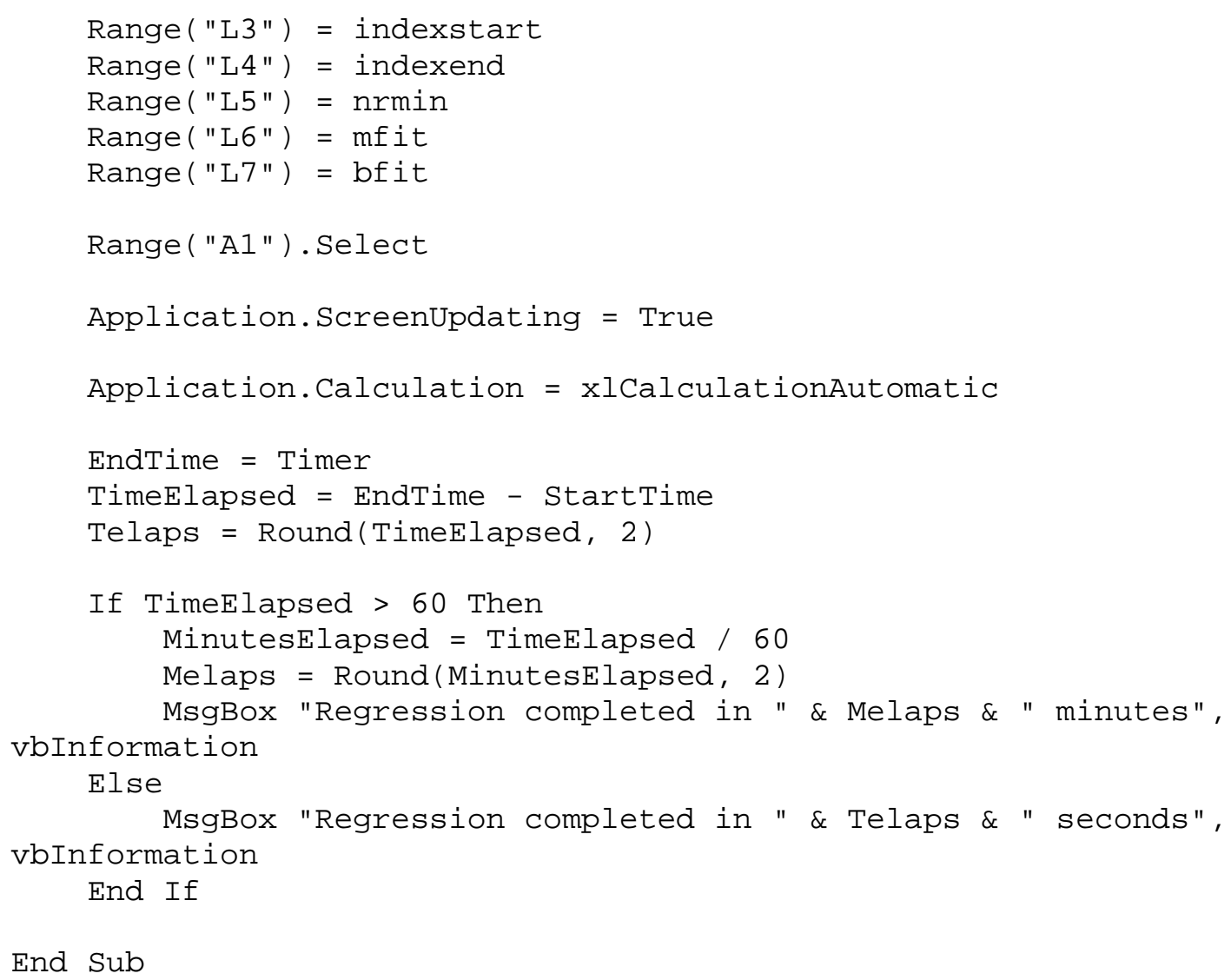

